\title{
On a class of multiscale cancer cell migration models: Well-posedness in less regular function spaces
}

\author{
Thomas Lorenz ${ }^{1}$ and Christina Surulescu ${ }^{2}$
}

\begin{abstract}
The aim is to prove global existence and uniqueness of square integrable solutions to a class of multiscale models for tumour cell migration involving chemotaxis, haptotaxis, and subcellular dynamics. This approach allows the tissue fibre and cell densities as well as concentrations of chemotactic signals to be less regular and the conditions sufficient for well-posedness of the multiscale model to be less restrictive than in previous settings.
\end{abstract}

\section{Introduction}

Tumour cell invasion is one of the essential stages in the development of cancer. Thereby, the tumour cells migrate through the surrounding tissue (extracellular matrix) towards blood or lymph vessels which they penetrate and thus access the blood flow. Through the blood circulation they are taken to distant locations, where they extravasate and develop new tumours, a process known as metastasis $[23,58]$. The intravasation and the subsequent systemic spread of cancer cells is facilitated by chemotaxis, a complex process during which a gradient of extracellular compounds is detected by the cells and initiates a series of intracellular signalling pathways leading to the coordination of cell movement along the chemoattractant gradient $[18,42]$.

During their movement the tumour cells interact with the extracellular matrix (ECM): They need to rely on the tissue fibres, thus the latter enhance invasion upon providing a moving support, however they can also impede the motion when they are too dense and/or have an unfavourable orientation w.r.t. the cell's direction of migration. The latter situation often has as consequence a particular type of invasiveness termed mesenchymal (see, e.g., [28]). This migration phenotype is tightly connected with the production of proteinases (matrix degrading enzymes like matrix metalloproteinases MMP and cathepsins), which are upregulated in tumour cells $[34,37,52]$ and facilitate dissemination and metastasis [51, 52] upon allowing to remodel the ECM and to control its interaction with the cancer cells. A further effect of the proteolytic activity of matrix degradation is the production of chemotactic ECM fragments, as well as promigratory neoepitopes that engage specific sets of cell surface receptors (called integrins) $[28,29]$, these in turn triggering cell motility.

Hence, cancer cell invasion is a multiscale process ranging from the subcellular level of integrin binding and signalling pathways through the level of individual cell behaviour up to the scale of the entire cancer cell population. According to these scales, most of the available mathematical models can be assigned to three large classes:

\footnotetext{
${ }^{1}$ Institute of Mathematics, Goethe University Frankfurt am Main, e-mail: lorenz@math.uni-frankfurt.de

${ }^{2}$ Felix Klein Center of Mathematics, TU Kaiserslautern, Paul-Ehrlich-Str. 31, D-67663 Kaiserslautern,

Germany, e-mail: surulescu@mathematik.uni-kl.de, Wednesday $6^{\text {th }}$ March, 2013
} 
Microscopic models focus on processes at the intracellular and/or cell surface level, usually characterised with the aid of a system of ordinary differential equations for the concentration of the biochemical substances playing a role in the respective signalling pathways or characterising the evolution of integrin-ligand complexes actually initiating the cell response to its surroundings. We refer to, e.g., [10] for an emphasis on proteolysis, to [39] for the dependence of the cell migration speed on receptor and ligand densities and receptor-ligand binding constants, and to [44] for an investigation of space-time dynamics describing the onset of lamellipod protrusion (a crucial step in integrin-mediated haptotactic cell invasion).

In the mesoscopic framework the migration process is modelled by way of a kinetic transport equation for the cell population density, where an integral operator describing the changes in the cell velocity replaces the typical collision terms. This type of models was proposed by Othmer et al. [49] in order to characterise the so-called velocity jump dispersal of organisms like bacteria under a chemotactic signal. Rigorous theoretical results for a one-particle distribution function were provided by Stroock [53]. In the context of tumour cell migration, Hillen proposed a related model for mesenchymal invasion [32], which was further enlarged by Chauvière et al. [17] to account for chemotaxis and cell-cell interactions. The global existence of measure-valued solutions to such a kinetic model was proved by Hillen et al. [33] more recently.

Starting from mesoscopic models of the type mentioned above, one can derive macroscopic descriptions via adequate scalings and averaging, leading to evolution equations for the moments of cell distribution function, see, e.g., [24] for hyperbolic models for chemosensitive movement or [32] for parabolic and hyperbolic scalings in the context of mesenchymal cancer cell migration through network tissues. Rigorous results on hyperbolic and parabolic limits of kinetic equations for chemotaxis are presented, e.g., in [15] and [50], respectively. Further macroscopic models for cell migration directly rely on mass conservation and/or mechanical force balance $[6,16]$ or on the theory of mixtures $[8,43,61,60]$.

Multiscale settings interconnecting the modelling on the cell scale level with population level descriptions such as kinetic or macroscopic equations offer a deeper insight into the migratory mechanism and hence greatly enhance the realism of the models, but are rather scarce, due to their complexity. A promising multiscale model in the context of tumour immune system competition with medically induced activation-deactivation was proposed by Firmani, Guerri and Preziosi [25]. The same idea was retaken by Erban and Othmer [20] in a more recent model dealing with a multiscale description of bacterial chemotaxis, in which the relevant intracellular signal transduction was modelled by a simple excitation-adaptation mechanism, while the density function of bacteria obeys a Boltzmann-like equation coupled to a reactiondiffusion equation for the chemotactic signal. This model was enhanced later by Bournaveas and Calvez [12] in order to allow for true excitation in response to small perturbations. The global existence of its unique solution was shown in higher dimensions (a 1D result for the model in [20] can be found in [21]) via dispersion and Strichartz estimates under some borderline growth assumptions on the turning kernel for the velocity changes. A related, but more detailed multiscale model for bacterial dispersal, along with a different proof for the global existence of a unique solution was provided in [54]. The latter also introduced an equation-free model for 
the velocity jump movement of bacteria under the influence of a chemoattractant signal and of the intracellular dynamics. Furthermore numerical simulations for the macroscopic cell density are presented via a nonparametric method (see $[55,56]$ ), an issue of particular interest, since no reliable numerical methods are available so far for handling a full multiscale setting of this type. In [36], the authors started from the mesoscopic framework to deduce a multiscale model describing the evolution of tumour cell population density, whereby at the microscopic scale they did not account for genuine intracellular signalling, but rather for the receptor dynamics on the cell surface. Those integrins provided linkages to the tissue fibres and to the ECM fragments resulting from proteolytic degradation. The latter acted as a chemoattractant, the evolution of which was characterised by a reaction-diffusion equation connected to the kinetic equation for the cell density and to the cell surface dynamics of integrins. In [35], the model also accounted for the dependency of proteolytic cutting on the fibre density in the direction of movement. Those concrete settings both align to the very general kinetic theory of active particles (KTAP) proposed by Bellomo et al. [9]. Another class of multiscale models connecting the microscopic and macroscopic levels of cancer cell migration was considered in [57] and [46], where the focus was on the effect of heat shock proteins on the tumour cell motility with a less detailed, rather phenomenological description of the microscopic phenomena.

It is the model in [36] which makes the object of the present study. The existence of a unique weak solution to that coupled system was proved there in $L^{\infty}\left(0, T ; L^{1} \cap L^{\infty}\right.$ ) (for tumour cell density and fibre density) and respectively in $L^{\infty}\left(0, T ; L^{\infty} \cap W^{1,1}\right)$ (for the concentration of the proteolytic product) with the aid of a regularisation and a fixed point argument. The existence proof relies on the regularity assumption $L^{\infty}$ (over the entire, particularly unbounded, space of positions, velocities and internal states), which is used for the estimates in [36]. To the best of our knowledge, essential boundedness is encountered throughout all existence results to be found in literature for multiscale models of this type.

However, as cell migration is a highly erratic process featured by very complex phenomena and influenced by a strongly heterogeneous and rapidly modifying environment, the cell and fibre densities - though existing globally in time - should be allowed being less regular and the conditions for their existence being less restrictive. This requirement is fulfilled by our global existence and uniqueness result in $L^{2}$ spaces.

The paper is organised as follows: In Section 2, we recall the model introduced in [36], which will be subsequently analysed. The main results are stated in Section 3. They concern the differential equations with functional dependencies on the components. This generalization is to facilitate their application to future extensions of the cell migration model. Its proof relies on the method of successive approximations and, its structure is outlined in Section 4. Thereby, the coupled system is split into several subproblems: One for the cell density (Section 5) and two others for the fibre density and the concentration of proteolytic fragments acting as a chemoattractant respectively (Section 6). Finally, the fixed point argument in Section 7 will complete the existence proof of the full original problem. This method also leads us to sufficient conditions for the continuous dependence of the solution on the given data (i.e., initial states and coefficients) and for preserving nonnegativity of all concentrations and densities involved. 


\section{Setting of the problem}

\subsection{Model variables}

In order to ease the reading, we provide a summary of the variables involved in our model:

- $\mathbf{x} \in \mathbb{R}^{n}$ spatial position of cell;

- $\mathbf{v}=\bigcup_{\lambda \in\left[s_{1}, s_{2}\right]} \lambda \cdot \mathbb{S}^{n-1}=V \subseteq \mathbb{R}^{n}, 0 \leq s_{1}<s_{2}<\infty$ velocity; $\hat{\mathbf{v}}=\frac{\mathbf{v}}{|\mathbf{v}|}$ normalised velocity;

- $\mathbf{y}=\left(y_{1}, y_{2}\right) \in Y \subseteq \mathbb{R}^{d}$ vector characterising the integrin state on the cell surface;

- $y_{1}$ : concentration of a cell's integrins bound to ECM fibres;

- $y_{2}$ : concentration of a cell's integrins bound to the proteolytic product $L$;

- $R_{0}$ : total concentration of integrins (bound or unbound) in a cell;

- $\mathbf{z}:=(\mathbf{x}, \mathbf{v}, \mathbf{y}) \in \mathbb{R}^{n} \times V \times Y=: Z$ (just for abbreviating the tuples)

- $\theta \in \mathbb{S}^{n-1}$ orientation of the ECM fibres;

- $f:[0, T] \longrightarrow L^{2}\left(\mathbb{R}^{n} \times V \times Y\right)$ time-dependent cell concentration (Lebesgue density);

- $\bar{f}(t, \mathbf{x})$ : macroscopic cancer cell density;

- $Q:[0, T] \longrightarrow L^{2}\left(\mathbb{R}^{n} \times \mathbb{S}^{n-1}\right)$ time-dependent density of ECM fibres;

- $\bar{Q}(t, \mathbf{x})$ : macroscopic density of ECM fibres;

- $L:] 0, T] \longrightarrow W^{1,2}\left(\mathbb{R}^{n}\right)$ time-dependent spatial concentration of chemoattractant (ECM fragments resulted by proteolytic degradation);

- isotropic diffusion $\kappa_{L}>0$ of chemoattractant.

Throughout this work we will use the same notation for the species $\bar{Q}$ and $L$ and for their densities and concentrations, respectively.

\subsection{Microscopic dynamics of migrating cells}

Let $\mathbb{S}^{n-1}$ denote the unit sphere in $\mathbb{R}^{n}$ and let $\theta \in \mathbb{S}^{n-1}$ be the fibre orientation. Then we denote the density of ECM fibres oriented in the direction $\theta$ at time $t$ and at location $\mathbf{x} \in \mathbb{R}^{n}$ by $Q(t, \mathbf{x}, \theta)$. The total concentration of ECM molecules is then given by

$$
\bar{Q}(t, \mathbf{x}):=\int_{\mathbb{S}^{n-1}} Q(t, \mathbf{x}, \theta) d \theta
$$


Let $V \subseteq \mathbb{R}^{n}$ represent the set of all possible velocities of moving cells. We assume that $V$ is radially symmetric and can be written as

$$
V=\left[s_{1}, s_{2}\right] \cdot \mathbb{S}^{n-1} \stackrel{\text { Def. }}{=} \bigcup_{\lambda \in\left[s_{1}, s_{2}\right]} \lambda \cdot \mathbb{S}^{n-1} \subseteq \mathbb{R}^{n}, \quad 0 \leq s_{1}<s_{2}<\infty,
$$

where $\left[s_{1}, s_{2}\right]$ is the range of possible speeds. ${ }^{3}$ We consider the population of cells as a system of $\mathrm{N}$ particles having positions $\mathbf{x}^{j} \in \mathbb{R}^{n}$ and velocities $\mathbf{v}^{j} \in V$ for $j=1, \ldots, N$. In the absence of reorientation and assuming that there are no external forces, the cells move along straight lines obeying Newton's first law of motion

$$
\begin{aligned}
\frac{d \mathbf{x}^{j}}{d t} & =\mathbf{v}^{j} \\
\frac{d \mathbf{v}^{j}}{d t} & =0 .
\end{aligned}
$$

For the dynamics on the cell surface, we use a kinetic model for the binding of ECM-proteins $\bar{Q}$ and proteolytic product $L$ to free integrins denoted by $R$. The reversible binding of integrins to ECM-proteins leads to a complex $R Q$ according to the equation

$$
\bar{Q}+R \underset{k_{-1}}{\stackrel{k_{1}}{\rightleftharpoons}} R Q .
$$

The corresponding equation for the formation and dissociation of complexes $R L$ of integrin and proteolytic product reads

$$
L+R \underset{k_{-2}}{\stackrel{k_{2}}{\rightleftharpoons}} R L
$$

We denote the concentrations of integrins of cell $j$ bound to ECM-molecules by $y_{1}^{j}$ and the concentration of integrins of the same cell bound to the proteolytic product $L$ by $y_{2}^{j}$. The total concentration of integrins (bound or unbound) of each cell is conserved and given by $R_{0} \in \mathbb{R}_{+}$. Thus, $R_{0}-y_{1}^{j}-y_{2}^{j}$ is the concentration of unbound integrins of cell $j$. Clearly, one has $\left(y_{1}^{j}, y_{2}^{j}\right) \in Y$ with

$$
Y:=\left\{\left(y_{1}, y_{2}\right) \in\left(0, R_{0}\right)^{2} \mid y_{1}+y_{2}<R_{0}\right\} .
$$

The state equations for the cell surface dynamics now read

$$
\frac{\partial \mathbf{y}^{j}}{\partial t}=\mathbf{G}\left(\mathbf{y}^{j}, \bar{Q}\left(t, \mathbf{x}^{j}\right), L\left(t, \mathbf{x}^{j}\right)\right)
$$

for $j=1, \ldots, N$ and with the mapping $\mathbf{G}: Y \times[0, \infty) \times[0, \infty) \rightarrow \mathbb{R}^{2}$ defined by

$$
\mathbf{G}(\mathbf{y}, q, l):=\left(\begin{array}{c}
k_{1}\left(R_{0}-y_{1}-y_{2}\right) q-k_{-1} y_{1} \\
k_{2}\left(R_{0}-y_{1}-y_{2}\right) l-k_{-2} y_{2}
\end{array}\right) .
$$

This system relying on mass action kinetics characterises the subcellular dynamics in $[35,36]$. Here we consider a slightly different model and assume the bindings between integrins and ECM fibres and proteolytic residuals to infer a certain amount of saturation:

$$
\mathbf{G}(\mathbf{y}, q, l):=\left(\begin{array}{l}
k_{1}\left(R_{0}-y_{1}-y_{2}\right) \frac{q}{1+\gamma_{Q} q}-k_{-1} y_{1} \\
k_{2}\left(R_{0}-y_{1}-y_{2}\right) \frac{l}{1+\gamma_{L} l}-k_{-2} y_{2}
\end{array}\right)
$$

with some positive saturation constants $\gamma_{Q}$ and $\gamma_{L}$.

\footnotetext{
${ }^{3}$ Hence the case with directed tissue fibres is allowed as well.
} 
This choice corresponds to the assumption of a constant number of cell surface receptors, hence limited binding possibilities. The same motivation applies in Subsection 2.3 below to the interaction between cells and ECM tissue fibres in the haptotaxis operators $\mathcal{H}_{+}$and $\mathcal{H}_{-}$ (see (8),(9)) and the equations (18),(19) for the evolution of fibre density and concentration of proteolytic rests, respectively.

Remark 2.1 Observe that the set $Y$ defined in (4) is not closed, since this would mean that (at least) one of the following situations occurs:

- there is no complex $R Q$, thus no binding between ECM and integrins, which is very improbable, since the cell moves in the tissue and therefore has to rely on the ECM fibres;

- there is no complex $R L$, thus no binding between integrins and proteolytic rests of the ECM, which is again highly improbable, because of the quasi-ubiquity of these rests. Moreover, no binding to $L$ would mean no chemotaxis;

- all receptors are occupied by ECM and/or proteolytic rests: this actually never happens and has never been observed, since this would impede chemotactic behaviour and migration per se.

Furthermore, considering such an open and bounded set requires invariance properties for the flow of (5), see [36]. In order to present the existence proof in a more transparent way, we start with the additional assumption $Y=\mathbb{R}^{d}$. Furthermore, our considerations will be carried out for general dimensions $d$ and $n$ although only $d=2$ and $n=3$ are needed for the problem at hand.

\subsection{Mesoscopic model. Including the microscopic dynamics}

Next we propose a multiscale model involving the mesoscale dynamics of the population density $f(t, \mathbf{x}, \mathbf{v}, \mathbf{y})$ of cells that have velocity vector $\mathbf{v}$ and - on the microscale - the internal state ${ }^{4} \mathbf{y}$ at time $t$ and location $\mathbf{x}$. The model (a deduction of which can be found in [36]) is completed with the equations for the ECM fibre density $Q(t, \mathbf{x}, \theta)$ and for the concentration of the proteolytic product $L$.

In the framework of our model for tumour cell migration, the changes in velocity orientation can occur due to one of the following two kinds of events:

- A cell may encounter a collagen fibre of the ECM and adapt its motion according to the direction of this fibre. We model this with a haptotactic term $\mathcal{H}(f, Q, \mathbf{x}, \mathbf{y})$.

- A cell may adjust its orientation to the gradient of the attracting chemical $L$, leading to a chemotactic term $\mathcal{C}(f, L, \mathbf{x}, \mathbf{y})$.

\footnotetext{
${ }^{4}$ As in [36], we (slightly abusively) call the integrin dynamics on the cell surface internal dynamics, since receptor bindings are the first step in the downstream intracellular events conditioning the motile behaviour.
} 
We assume that the probability of a cell changing its orientation in the time interval under consideration is proportional to $\Delta t$ and denote the corresponding rates by $p_{h}(t, \mathbf{x}, \mathbf{v}, \mathbf{y})$ and $p_{c}(t, \mathbf{x}, \mathbf{v}, \mathbf{y})$. The operator for haptotaxis $\mathcal{H}$ can be written as $\mathcal{H}=\mathcal{H}_{+}-\mathcal{H}_{-}$with a gain term $\mathcal{H}_{+}$and a loss term $\mathcal{H}_{-}$which (using the shorthand notation $f\left(\mathbf{v}^{\prime}\right):=f\left(t, \mathbf{x}, \mathbf{v}^{\prime}, \mathbf{y}\right)$ and $\left.Q\left(\theta^{\prime}\right):=Q\left(t, \mathbf{x}, \theta^{\prime}\right)\right)$ are defined as

$$
\begin{aligned}
\mathcal{H}_{+}(f, Q, \mathbf{x}, \mathbf{y}) & =\int_{V} \int_{\mathbb{S}^{n-1}} p_{h}\left(t, \mathbf{x}, \mathbf{v}^{\prime}, \mathbf{y}\right) \psi\left(\mathbf{v} ; \mathbf{v}^{\prime}, \theta^{\prime}\right) f\left(\mathbf{v}^{\prime}\right) \frac{Q\left(\mathbf{x}, \theta^{\prime}\right)}{1+\gamma_{Q} \cdot\left|Q\left(\mathbf{x}, \theta^{\prime}\right)\right|} d \theta^{\prime} d \mathbf{v}^{\prime} \\
\mathcal{H}_{-}(f, Q, \mathbf{x}, \mathbf{y}) & =f(\mathbf{v}) p_{h}(t, \mathbf{x}, \mathbf{v}, \mathbf{y}) \cdot \int_{V} \int_{\mathbb{S}^{n-1}} \psi\left(\mathbf{v}^{\prime} ; \mathbf{v}, \theta^{\prime}\right) \frac{Q\left(\mathbf{x}, \theta^{\prime}\right)}{1+\gamma_{Q} \cdot\left|Q\left(\mathbf{x}, \theta^{\prime}\right)\right|} d \theta^{\prime} d \mathbf{v}^{\prime}
\end{aligned}
$$

where $\psi\left(\mathbf{v} ; \mathbf{v}^{\prime}, \theta^{\prime}\right)$ denotes the likelihood of a cell having the velocity $\mathbf{v}^{\prime}$ before the encounter with a fibre having orientation $\theta^{\prime}$ to continue its motion with the velocity $\mathbf{v}$ after the interaction. Since the cells are conserved during interactions with the fibres, the following condition must hold:

$$
\int_{V} \psi\left(\mathbf{v} ; \mathbf{v}^{\prime}, \theta^{\prime}\right) d \mathbf{v}=1
$$

A possible choice for the haptotactic turning kernel $^{5}$ could be

$$
\psi\left(\mathbf{v} ; \mathbf{v}^{\prime}, \theta^{\prime}\right)=\left|\hat{\mathbf{v}}^{\prime} \cdot \theta^{\prime}\right| K_{H}^{(1)}\left(\mathbf{v}, \theta^{\prime}\right)+\left(1-\left|\hat{\mathbf{v}}^{\prime} \cdot \theta^{\prime}\right|\right) K_{H}^{(2)}\left(\mathbf{v}, \mathbf{v}^{\prime}\right),
$$

where $K_{H}^{(1)}$ and $K_{H}^{(2)}$ denote two reorientation kernels. The fibre orientation assigns different weights to each of these kernels. Hence, if the encountered fibres are nearly orthogonal to the cell's previous direction of motion, then the probability of cutting these fibres by proteolysis is high and the kernel $K_{H}^{(2)}$ will be preferentially chosen. The latter could be, e.g., a Gaussian kernel ensuring that the new direction of the cell stays close to the old one, while the choice for $K_{H}^{(1)}$ can be for instance the Dirac distribution $\delta\left(\mathbf{v}-\theta^{\prime}\right)$, expressing the fact that the new cell's direction is dictated by the direction of the encountered fibre(s).

The decomposition $\mathcal{C}=\mathcal{C}_{+}-\mathcal{C}_{-}$for the operator related to chemotaxis $\mathcal{C}$ into a gain term and a loss term reads

$$
\begin{aligned}
& \mathcal{C}_{+}(f, L, \mathbf{x}, \mathbf{y})=\int_{V} p_{c}\left(t, \mathbf{x}, \mathbf{v}^{\prime}, \mathbf{y}\right) K[L]\left(\mathbf{v} ; \mathbf{v}^{\prime}, \mathbf{x}, \mathbf{y}\right) f\left(\mathbf{v}^{\prime}\right) d \mathbf{v}^{\prime} \\
& \mathcal{C}_{-}(f, L, \mathbf{x}, \mathbf{y})=p_{c}(t, \mathbf{x}, \mathbf{v}, \mathbf{y}) f(\mathbf{v}) .
\end{aligned}
$$

The turning kernel is given by the mixture

$$
K[L]\left(\mathbf{v} ; \mathbf{v}^{\prime}, \mathbf{x}, \mathbf{y}\right)=\alpha_{1}(\mathbf{y}) K\left(\mathbf{v}, \mathbf{v}^{\prime}\right)+\alpha_{2}(\mathbf{y}) K(\mathbf{v}, \nabla L),
$$

with the coefficients $\alpha_{1}, \alpha_{2}: Y \rightarrow[0,1]$ assigning corresponding weights to the random direction change and to the chemotactic effects, respectively, and such that $\alpha_{1}+\alpha_{2}=1$ on $Y$. Thereby, $K$ can be any kernel satisfying the conservation condition

$$
\int_{V} K\left(\mathbf{v}, \mathbf{v}^{\prime}\right) d \mathbf{v}=1
$$

\footnotetext{
${ }^{5}$ In [36] it has been kept in a generic form and it assumed the alignment of the tumour cell to the encountered fibres, not accommodating in its structure the effects of proteolysis
} 


\section{Remark 2.2}

- One could consider two different kernels as well, one for the turning and one for the chemotactic part. We chose here the same kernel just for the sake of simplicity.

- A possible choice for the weights $\alpha_{i}(i=1,2)$ is $\alpha_{i}(\mathbf{y})=\frac{\tilde{\alpha}_{i}(\mathbf{y})}{\tilde{\alpha}_{1}(\mathbf{y})+\tilde{\alpha}_{2}(\mathbf{y})}$, with $\tilde{\alpha}_{i}(\mathbf{y})=$ $\exp \left(C_{i}|\mathbf{y}|\right)$ and constants $C_{i}$ such that $C_{1} C_{2}<0$.

Hence we obtain for the tumour cell density $f$ the following transport equation:

$$
\frac{\partial f}{\partial t}+\mathbf{v} \cdot \nabla_{\mathbf{x}} f+\nabla_{\mathbf{y}} \cdot(\mathbf{G}(\mathbf{y}, \bar{Q}, L) f)=\mathcal{H}(f, Q)+\mathcal{C}(f, L)
$$

The macroscopic population density at time $t$ and position $\mathbf{x}$ is obtained by integration over all possible velocities and internal states

$$
\bar{f}(t, \mathbf{x}):=\int_{Y} \int_{V} f(t, \mathbf{x}, \mathbf{v}, \mathbf{y}) d \mathbf{v} d \mathbf{y} .
$$

We define the mean projection of movement direction on the fibre orientation:

$$
\Pi[f](t, \mathbf{x}, \theta):=\frac{1}{\bar{f}(t, \mathbf{x})} \cdot \int_{Y} \int_{V}|\theta \cdot \hat{\mathbf{v}}| f(t, \mathbf{x}, \mathbf{v}, \mathbf{y}) d \mathbf{v} d \mathbf{y}
$$

where $\hat{\mathbf{v}}$ is a notation for $\frac{\mathbf{v}}{|\mathbf{v}|}$. For the tissue modification model we consider the following evolution equation for the fibre density $Q(t, \mathbf{x}, \theta)$ analogously to [32]:

$$
\frac{\partial Q}{\partial t}=r_{\mathrm{ECM}}(\Pi[f](t, \mathbf{x}, \theta)-1) \bar{f}(t, \mathbf{x}) \frac{Q(\mathbf{x}, \theta)}{1+\gamma_{Q} \cdot|Q(\mathbf{x}, \theta)|}
$$

where $r_{\mathrm{ECM}}$ is the degradation rate of ECM fibres by the action of matrix degrading enzymes. The reaction-diffusion equation for the product $L(t, \mathbf{x})$ of proteolysis reads

$$
\frac{\partial L}{\partial t}=\kappa_{L} \cdot \Delta_{\mathbf{x}} L+\int_{\mathbb{S}^{n-1}} r_{\mathrm{ECM}}(1-\Pi[f](t, \mathbf{x}, \theta)) \bar{f}(t, \mathbf{x}) \frac{Q(\mathbf{x}, \theta)}{1+\gamma_{Q} \cdot|Q(\mathbf{x}, \theta)|} d \theta-r_{L} \cdot L
$$

where $\kappa_{L}>0$ is the diffusion coefficient and $r_{L} \geq 0$ the decay rate of $L$. The production term in (19) is just the degradation term in (18) integrated over all directions $\theta$ and with a negative sign, i.e. fibres (the density of which is denoted by $Q$ ) are degraded into fragments (of concentration $L$ ).

\section{The main results about functional equations}

Definition 3.1 Set $Z \stackrel{\text { Def. }}{=} \mathbb{R}^{n} \times V \times Y$ and define $d^{f}: L^{2}(Z) \times L^{2}(Z) \longrightarrow[0, \infty[$ as

$$
\begin{aligned}
d^{f}(f, g):=\sup \left\{\int_{Z} \varphi \cdot(f-g) d \mathbf{z} \mid\right. & \varphi \in C_{c}^{0}(Z), \quad \nabla_{\mathbf{y}} \varphi \in L^{\infty}\left(Z, \mathbb{R}^{d}\right), \\
& \left.\|\varphi\|_{L^{2}} \leq 1,\|\varphi\|_{L^{\infty}} \leq 1,\left\|\nabla_{\mathbf{y}} \varphi\right\|_{L^{\infty}} \leq 1\right\} .
\end{aligned}
$$


Theorem 3.2 (Existence and uniqueness of solution on $Z=\mathbb{R}^{n} \times V \times \mathbb{R}^{d}$ )

Consider the system of nonlinear functional differential equations

$$
\left\{\begin{aligned}
\partial_{t} f+\nabla_{\mathbf{x}} \cdot(\mathbf{v} f)+\nabla_{\mathbf{y}} \cdot(\mathcal{G}(t, f, Q, L) f) & =\mathcal{U}(t, f, Q, L) \cdot f+\mathcal{W}(t, f, Q, L) \\
\partial_{t} Q & =\mathcal{T}(t, f, Q, L) \\
\partial_{t} L & =\kappa_{L} \cdot \Delta_{\mathbf{x}} L+\mathcal{R}(t, f, Q, L)
\end{aligned}\right.
$$

with $Y=\mathbb{R}^{d}$, a constant $\kappa_{L}>0$ and the coefficient functions

$$
\begin{aligned}
& \mathcal{G}:[0, T] \times L^{2}(Z) \times L^{2}\left(\mathbb{R}^{n} \times \mathbb{S}^{n-1}\right) \times W^{1,2}\left(\mathbb{R}^{n}\right) \longrightarrow\left\{\mathbf{g} \in L^{\infty}\left(Z, \mathbb{R}^{d}\right) \cap L^{2} \mid \partial_{\mathbf{y}} \mathbf{g} \in L^{\infty}\right\}, \\
& \mathcal{U}:[0, T] \times L^{2}(Z) \times L^{2}\left(\mathbb{R}^{n} \times \mathbb{S}^{n-1}\right) \times W^{1,2}\left(\mathbb{R}^{n}\right) \longrightarrow\left\{u \in L^{\infty}(Z) \cap L^{2}(Z) \mid \nabla_{\mathbf{y}} u \in L^{\infty}\right\}, \\
& \mathcal{W}:[0, T] \times L^{2}(Z) \times L^{2}\left(\mathbb{R}^{n} \times \mathbb{S}^{n-1}\right) \times W^{1,2}\left(\mathbb{R}^{n}\right) \longrightarrow L^{2}(Z), \\
& \mathcal{R}:[0, T] \times L^{2}(Z) \times L^{2}\left(\mathbb{R}^{n} \times \mathbb{S}^{n-1}\right) \times W^{1,2}\left(\mathbb{R}^{n}\right) \longrightarrow L^{2}\left(\mathbb{R}^{n}\right), \\
& \mathcal{T}:[0, T] \times L^{2}(Z) \times L^{2}\left(\mathbb{R}^{n} \times \mathbb{S}^{n-1}\right) \times W^{1,2}\left(\mathbb{R}^{n}\right) \longrightarrow L^{2}\left(\mathbb{R}^{n} \times \mathbb{S}^{n-1}\right)
\end{aligned}
$$

under the following assumptions:

(i) (Global a priori bounds and growth conditions)

$$
\sup _{t, f, Q, L}\left(\left\|\operatorname{div}_{\mathbf{y}} \mathcal{G}(t, f, Q, L)\right\|_{L^{\infty}(Z)}+\|\mathcal{U}(t, f, Q, L)\|_{L^{\infty}(Z)}+\frac{\|\mathcal{W}(t, f, Q, L)\|_{L^{2}(Z)}}{1+\|f\|_{L^{2}(Z)}}\right)<\infty
$$

(ii) (Locally uniform a priori bounds and growth conditions)

For every $\rho>0$, there exists a constant $C_{\rho}<\infty$ such that for all $t, \widehat{f}, Q, \widehat{Q}, L, \widehat{L}$ with $\|\widehat{f}\|_{L^{2}(Z)} \leq \rho,\|\widehat{Q}\|_{L^{2}\left(\mathbb{R}^{n} \times \mathbb{S}^{n-1}\right)} \leq \rho$ and $\|\widehat{L}\|_{W^{1,2}\left(\mathbb{R}^{n}\right)} \leq \rho \cdot\left(1+t^{-1 / 2}\right)$, it holds

$$
\left\{\begin{aligned}
\|\mathcal{T}(t, \widehat{f}, Q, L)\|_{L^{2}\left(\mathbb{R}^{n} \times \mathbb{S}^{n-1}\right)} & \leq C_{\rho} \cdot\left(1+\|Q\|_{L^{2}\left(\mathbb{R}^{n} \times \mathbb{S}^{n-1}\right)}\right) \\
\|\mathcal{R}(t, \widehat{f}, \widehat{Q}, L)\|_{L^{2}\left(\mathbb{R}^{n}\right)} & \leq C_{\rho} \cdot\left(1+\|L\|_{L^{2}\left(\mathbb{R}^{n}\right)}\right) \\
\|\mathcal{G}(t, \widehat{f}, \widehat{Q}, \widehat{L})\|_{L^{\infty}\left(\mathbb{R}^{n}\right)}+\left\|\partial_{\mathbf{y}} \mathcal{G}(t, \widehat{f}, \widehat{Q}, \widehat{L})\right\|_{L^{\infty}\left(\mathbb{R}^{n}, \mathbb{R}^{d \times d}\right)} & \leq C_{\rho} \\
\left\|\nabla_{\mathbf{y}} \mathcal{U}(t, \widehat{f}, \widehat{Q}, \widehat{L})\right\|_{L^{\infty}\left(\mathbb{R}^{n}, \mathbb{R}^{d}\right)} & \leq C_{\rho}
\end{aligned}\right.
$$

(iii) $\mathcal{G}, \mathcal{U}, \mathcal{W}, \mathcal{R}, \mathcal{T}:[0, T] \times\left(L^{2}(Z), d^{f}\right) \times L^{2}\left(\mathbb{R}^{n} \times \mathbb{S}^{n-1}\right) \times W^{1,2}\left(\mathbb{R}^{n}\right) \longrightarrow\left(L^{2},\|\cdot\|_{L^{2}}\right)$ are measurable.

(iv) (Locally uniform Lipschitz conditions w.r.t. states)

For every $\rho>0$, there exists a constant $\Lambda_{\rho}>0$ such that for all $t, f_{1}, f_{2}, Q_{1}, Q_{2}, L_{1}, L_{2}$ with $\sup _{j=1,2}\left\{\left\|f_{j}\right\|_{L^{2}(Z)},\left\|Q_{j}\right\|_{L^{2}\left(\mathbb{R}^{n} \times \mathbb{S}^{n-1}\right)}, \frac{1}{1+t^{-1 / 2}}\left\|L_{j}\right\|_{W^{1,2}\left(\mathbb{R}^{n}\right)}\right\} \leq \rho$, the conditions $\left\|\mathcal{R}\left(t, f_{1}, Q_{1}, L_{1}\right)-\mathcal{R}\left(t, f_{2}, Q_{2}, L_{2}\right)\right\|_{L^{2}} \leq \Lambda_{\rho}\left(d^{f}\left(f_{1}, f_{2}\right)+\left\|Q_{1}-Q_{2}\right\|_{L^{2}}+\left\|L_{1}-L_{2}\right\|_{L^{2}}\right)$, $\left\|\mathcal{F}\left(t, f_{1}, Q_{1}, L_{1}\right)-\mathcal{F}\left(t, f_{2}, Q_{2}, L_{2}\right)\right\|_{L^{2}} \leq \Lambda_{\rho}\left(d^{f}\left(f_{1}, f_{2}\right)+\left\|Q_{1}-Q_{2}\right\|_{L^{2}}+\left\|L_{1}-L_{2}\right\|_{W^{1,2}}\right)$ hold for each function $\mathcal{F}$ of the coefficients $\mathcal{G}, \mathcal{U}, \mathcal{W}, \mathcal{T}$.

$(v)$ (Locally uniform choice of function dominating values of $\mathcal{W}$ )

For every $\rho>0$, there exist $w_{\rho} \in L^{2}(Z)$ and compact sets $K_{\mathbf{x}} \subseteq \mathbb{R}^{n}, K_{\mathbf{y}} \subseteq Y=\mathbb{R}^{d}$ such that for all $t, f, Q, L$ with $\sup \left\{\|f\|_{L^{2}(Z)},\|Q\|_{L^{2}\left(\mathbb{R}^{n} \times \mathbb{S}^{n-1}\right)}, \frac{1}{1+t^{-1 / 2}}\|L\|_{W^{1,2}\left(\mathbb{R}^{n}\right)}\right\} \leq \rho$ :

$|\mathcal{W}(t, f, Q, L)| \leq w_{\rho}$ Lebesgue-almost everywhere in $Z \backslash\left(K_{\mathbf{x}} \times V \times K_{\mathbf{y}}\right)$. 
Then every initial value problem related to (20) has a unique weak solution $f:[0, T] \longrightarrow L^{2}(Z)$, $\left.\left.Q:[0, T] \longrightarrow L^{2}\left(\mathbb{R}^{n} \times \mathbb{S}^{n-1}\right), L:\right] 0, T\right] \longrightarrow W^{1,2}\left(\mathbb{R}^{n}\right)$. Moreover each component $f, Q, L$ is bounded w.r.t. the $L^{2}$ norm.

\section{Proposition 3.3 (Lipschitz continuous dependence of the solution on given data)}

For $Z \stackrel{\text { Def. }}{=} \mathbb{R}^{n} \times V \times \mathbb{R}^{d}$ and $j=1,2$, let $\mathcal{G}^{(j)}, \mathcal{U}^{(j)}, \mathcal{W}^{(j)}$ and $\mathcal{R}^{(j)}, \mathcal{T}^{(j)}$ satisfy the assumptions of Theorem 3.2 (with the same constants for a priori bounds and linear growth conditions). Moreover, $\left(f^{(1)}, Q^{(1)}, L^{(1)}\right)$ and $\left(f^{(2)}, Q^{(2)}, L^{(2)}\right)$ denote the unique solutions of system $(20)$ related to the coefficients $\mathcal{G}^{(j)}, \mathcal{U}^{(j)}, \mathcal{W}^{(j)}, \mathcal{R}^{(j)}, \mathcal{T}^{(j)}$ and the initial states $\left(f_{0}^{(j)}, Q_{0}^{(j)}, L_{0}^{(j)}\right) \in$ $L^{2}(Z) \times L^{2}\left(\mathbb{R}^{n} \times \mathbb{S}^{n-1}\right) \times L^{2}\left(\mathbb{R}^{n}\right), j=1,2$, respectively. Choose some $R>0$ with

$$
\max _{j=1,2}\left(\left\|f_{0}^{(j)}\right\|_{L^{2}(Z)}+\left\|Q_{0}^{(j)}\right\|_{L^{2}\left(\mathbb{R}^{n} \times \mathbb{S}^{n-1}\right)}+\left\|L_{0}^{(j)}\right\|_{L^{2}\left(\mathbb{R}^{n}\right)}\right) \leq R .
$$

Then there exists a constant $C>0$ depending on $R, T$ and the constants of coefficient functions (in assumptions $(i)-(i v))$ such that the "distance" function $\psi:[0, T] \longrightarrow[0, \infty[$,

$$
\psi(t):=d^{f}\left(f^{(1)}(t), f^{(2)}(t)\right)+\left\|Q^{(1)}(t)-Q^{(2)}(t)\right\|_{L^{2}\left(\mathbb{R}^{n} \times \mathbb{S}^{n-1}\right)}+\left\|L^{(1)}(t)-L^{(2)}(t)\right\|_{L^{2}\left(\mathbb{R}^{n}\right)}
$$

satisfies the following estimate for every $t \in[0, T]$

$$
\begin{aligned}
\psi(t) \leq e^{C t} \cdot\left(\psi(0) \cdot e^{C \sqrt{t}}+C \cdot t \cdot \sup \right. & \left(\left\|\mathcal{G}^{(1)}-\mathcal{G}^{(2)}\right\|_{L^{2}}+\left\|\mathcal{U}^{(1)}-\mathcal{U}^{(2)}\right\|_{L^{2}}+\left\|\mathcal{W}^{(1)}-\mathcal{W}^{(2)}\right\|_{L^{2}}\right. \\
+ & \left.\left.\left\|\mathcal{R}^{(1)}-\mathcal{R}^{(2)}\right\|_{L^{2}\left(\mathbb{R}^{n}\right)}+\left\|\mathcal{T}^{(1)}-\mathcal{T}^{(2)}\right\|_{L^{2}\left(\mathbb{R}^{n} \times \mathbb{S}^{n-1}\right)}\right)\right)
\end{aligned}
$$

Corollary 3.4 (Existence and uniqueness of solution on $Z=\mathbb{R}^{n} \times V \times Y, Y \subsetneq \mathbb{R}^{d}$ ) Let $Y \subsetneq \mathbb{R}^{d}$ now be a nonempty open subset with bounded Lipschitz boundary. In addition to the hypotheses $(i)-(v)$ of Theorem 3.2, suppose

(vi) For every $t \in[0, T], f \in L^{2}(Z), Q \in L^{2}\left(\mathbb{R}^{n} \times \mathbb{S}^{n-1}\right)$ and $L \in W^{1,2}\left(\mathbb{R}^{n}\right)$, the function $\mathcal{G}(t, f, Q, L) \in L^{\infty}\left(Z, \mathbb{R}^{d}\right)$ satisfies following tangential condition to the closure $\bar{Y} \subset \mathbb{R}^{d}$ : For every $\mathbf{x} \in \mathbb{R}^{n}, \mathbf{v} \in V$ and a.e. $t \in[0, T]$, the vector field $\mathcal{G}(t, f, Q, L)(\mathbf{x}, \mathbf{v}, \cdot) \in$ $W^{1, \infty}\left(Y, \mathbb{R}^{d}\right)$ has a Lipschitz continuous representative and so, a continuous extension to $\bar{Y}$. The latter is assumed to fulfil at each point $\mathbf{y} \in \bar{Y}$

$$
\liminf _{h \downarrow 0} \frac{1}{h} \cdot \operatorname{dist}(\mathbf{y}+h \cdot \mathcal{G}(t, f, Q, L)(\mathbf{x}, \mathbf{v}, \mathbf{y}), \bar{Y})=0 .
$$

Then every initial value problem related to (20) has a unique weak solution $f:[0, T] \longrightarrow L^{2}(Z)$, $\left.\left.Q:[0, T] \longrightarrow L^{2}\left(\mathbb{R}^{n} \times \mathbb{S}^{n-1}\right), L:\right] 0, T\right] \longrightarrow W^{1,2}\left(\mathbb{R}^{n}\right)$.

Moreover, assumption (21) about several initial states implies estimate (22).

\section{Corollary 3.5 (Preserving nonnegativity of components $f, L$ of solutions)}

Let $Y \subsetneq \mathbb{R}^{d}$ (again) be a nonempty open subset with bounded Lipschitz boundary, $\beta_{L} \geq 0$. In addition to hypotheses $(i)-(v)$ of Theorem 3.2 and assumption (vi) of Corollary 3.4, suppose for Lebesgue-almost every $t \in[0, T]$ : 
(vii) For all $f_{0}, f_{1} \in L^{2}(Z), Q_{1} \in L^{2}\left(\mathbb{R}^{n} \times \mathbb{S}^{n-1}\right)$ and $L_{1} \in W^{1,2}\left(\mathbb{R}^{n}\right)$ with $f_{1} \geq 0$ (a.e. in $Z$ ), the following conditions hold

$$
\begin{cases}\mathcal{W}\left(t, f_{0}, Q_{1}, L_{1}\right) \geq 0 & \text { a.e. in } Z \stackrel{\text { Def. }}{=} \mathbb{R}^{n} \times V \times Y \\ \mathcal{R}\left(t, f_{1}, Q_{1}, L_{1}\right) \geq-\beta_{L} \cdot L_{1} & \text { a.e. in } Z .\end{cases}
$$

If the initial states $f_{0} \in L^{2}(Z), L_{0} \in L^{2}\left(\mathbb{R}^{n}\right)$ satisfy the additional sign condition $f_{0} \geq 0$, $L_{0} \geq 0$, then so do the components $\left.\left.f:[0, T] \longrightarrow L^{2}(Z), L:\right] 0, T\right] \longrightarrow W^{1,2}\left(\mathbb{R}^{n}\right)$ of the uniquely determined weak solutions to problem (20) at every time instant $t \in] 0, T]$.

\section{Applying the general theorems to the model for cell migration}

The next step is to draw some conclusions about the following mesoscopic model for cell migration through tissue:

$$
\left\{\begin{aligned}
\partial_{t} f+\nabla_{\mathbf{x}} \cdot(\mathbf{v} f)+\nabla_{\mathbf{y}} \cdot(\mathcal{G}(t, f, Q, L) f) & =\mathcal{U}(t, f, Q, L) \cdot f+\mathcal{W}(t, f, Q, L) \\
\partial_{t} Q & =\mathcal{T}(t, f, Q, L) \\
\partial_{t} L & =\kappa_{L} \cdot \Delta_{\mathbf{x}} L+\mathcal{R}(t, f, Q, L)
\end{aligned}\right.
$$

with the open set $Y:=\left\{\left(y_{1}, y_{2}\right) \in\right] 0, R_{0}\left[^{2} \mid y_{1}+y_{2}<R_{0}\right\}$, the coefficients

$$
\begin{aligned}
& \left.\mathcal{G}(t, f, Q, L)\right|_{(\mathbf{x}, \mathbf{v}, \mathbf{y})}:=\left(\begin{array}{l}
k_{1}\left(R_{0}-y_{1}-y_{2}\right) \cdot \frac{|\bar{Q}(\mathbf{x})|}{1+\gamma_{Q} \cdot|Q(\mathbf{x})|}-k_{-1} \cdot y_{1} \\
k_{2}\left(R_{0}-y_{1}-y_{2}\right) \cdot \frac{|L(\mathbf{x})|}{1+\gamma_{L} \cdot|L(\mathbf{x})|}-k_{-2} \cdot y_{2}
\end{array}\right) \\
& \left.\mathcal{U}(t, f, Q, L)\right|_{(\mathbf{x}, \mathbf{v}, \mathbf{y})}:=\frac{1}{f(\mathbf{x}, \mathbf{v}, \mathbf{y})} \cdot\left(\mathcal{H}_{-}(f, Q, \mathbf{x}, \mathbf{y})+\mathcal{C}_{-}(f, L, \mathbf{x}, \mathbf{y})\right) \\
& =p_{h}(t, \mathbf{x}, \mathbf{v}, \mathbf{y}) \cdot \int_{V} \int_{\mathbb{S}^{n-1}} \psi\left(\mathbf{v}^{\prime} ; \mathbf{v}, \theta^{\prime}\right) \frac{Q\left(\mathbf{x}, \theta^{\prime}\right)}{1+\gamma_{Q} \cdot\left|Q\left(\mathbf{x}, \theta^{\prime}\right)\right|} d \theta^{\prime} d \mathbf{v}^{\prime}+p_{c}(t, \mathbf{x}, \mathbf{v}, \mathbf{y}) \\
& \left.\mathcal{W}(t, f, Q, L)\right|_{(\mathbf{x}, \mathbf{v}, \mathbf{y})}:=\mathcal{H}_{+}(f, Q, \mathbf{x}, \mathbf{y})+\mathcal{C}_{+}(f, L, \mathbf{x}, \mathbf{y}) \\
& =\int_{V} \int_{\mathbb{S}^{n-1}} p_{h}\left(t, \mathbf{x}, \mathbf{v}^{\prime}, \mathbf{y}\right) \psi\left(\mathbf{v} ; \mathbf{v}^{\prime}, \theta^{\prime}\right)\left|f\left(\mathbf{x}, \mathbf{v}^{\prime}, \mathbf{y}\right)\right| \frac{\left|Q\left(\mathbf{x}, \theta^{\prime}\right)\right|}{1+\gamma_{Q} \cdot\left|Q\left(\mathbf{x}, \theta^{\prime}\right)\right|} d \theta^{\prime} d \mathbf{v}^{\prime} \\
& +\int_{V} p_{c}\left(t, \mathbf{x}, \mathbf{v}^{\prime}, \mathbf{y}\right) \cdot K[L]\left(\mathbf{v} ; \mathbf{v}^{\prime}, \mathbf{x}, \mathbf{y}\right) \cdot\left|f\left(\mathbf{x}, \mathbf{v}^{\prime}, \mathbf{y}\right)\right| d \mathbf{v}^{\prime} \\
& \left.\mathcal{T}(t, f, Q, L)\right|_{(\mathbf{x}, \mathbf{v}, \mathbf{y})}:=r_{\mathrm{ECM}}(\Pi[f](\mathbf{x}, \theta)-1) \bar{f}(\mathbf{x}) \frac{Q(\mathbf{x}, \theta)}{1+\gamma_{Q} \cdot|Q(\mathbf{x}, \theta)|} \\
& =r_{\mathrm{ECM}} \int_{Y} \int_{V}\left(\left|\theta \cdot \hat{\mathbf{v}}^{\prime}\right|-1\right) f\left(\mathbf{x}, \mathbf{v}^{\prime}, \mathbf{y}^{\prime}\right) d \mathbf{v}^{\prime} d \mathbf{y}^{\prime} \cdot \frac{Q(\mathbf{x}, \theta)}{1+\gamma_{Q} \cdot|Q(\mathbf{x}, \theta)|} \\
& \left.\mathcal{R}(t, f, Q, L)\right|_{(\mathbf{x}, \mathbf{v}, \mathbf{y})}:=\int_{\mathbb{S}^{n-1}}\left(r_{\mathrm{ECM}} \int_{Y} \int_{V}\left(1-\left|\theta \cdot \hat{\mathbf{v}}^{\prime}\right|\right) f\left(\mathbf{x}, \mathbf{v}^{\prime}, \mathbf{y}^{\prime}\right) d \mathbf{v}^{\prime} d \mathbf{y}^{\prime} \frac{|Q(\mathbf{x}, \theta)|}{1+\gamma_{Q} \cdot|Q(\mathbf{x}, \theta)|}\right) d \theta \\
& -r_{L} \cdot L(\mathbf{x})
\end{aligned}
$$

and the notations

$$
\begin{aligned}
\bar{Q}(\mathbf{x}) & :=\int_{\mathbb{S}^{n-1}} Q(\mathbf{x}, \theta) d \theta \\
K[L]\left(\mathbf{v} ; \mathbf{v}^{\prime}, \mathbf{x}, \mathbf{y}\right) & :=\alpha_{1}(\mathbf{y}) \cdot K\left(\mathbf{v}, \mathbf{v}^{\prime}\right)+\alpha_{2}(\mathbf{y}) \cdot K\left(\mathbf{v}, \operatorname{proj}_{\overline{c o} V} \nabla L(\mathbf{x})\right),
\end{aligned}
$$


Now we specify the hypotheses for the parameter functions and constants which are to ensure existence, uniqueness and nonnegativity of weak solutions respectively. It is worth mentioning that whenever the components $f(t), Q(t), L(t)$ are surely nonnegative at every time instant then we can dispense with the absolute value in all quotient functions of the preceding model problem (23).

\section{Assumptions about this model}

(a) $\gamma_{L}>0, \gamma_{Q}>0, k_{ \pm 1} \geq 0, k_{ \pm 2} \geq 0, \kappa_{L}>0, r_{\mathrm{ECM}} \geq 0, r_{L} \geq 0$ $V \stackrel{\text { Def. }}{=}\left\{v \in \mathbb{R}^{n}\left|s_{1} \leq\right| v \mid \leq s_{2}\right\}$ with $0 \leq s_{1} \leq s_{2}<\infty$ (and so, $\overline{\text { co }} V=\overline{\mathbb{B}}_{s_{2}}(0) \subset \mathbb{R}^{n}$ )

(b) $p_{c}, p_{h} \in L^{\infty}\left(0, T ; L^{\infty}(Z)\right)$ have support in a joint compact subset of $Z \stackrel{\text { Def. }}{=} \mathbb{R}^{n} \times V \times Y$, $\partial_{\mathbf{y}} p_{c}, \partial_{\mathbf{y}} p_{h} \in L^{\infty}\left(0, T ; L^{\infty}\left(Z, \mathbb{R}^{d}\right)\right)$,

(c) $\psi \in L^{\infty}\left(V \times V \times \mathbb{S}^{n-1}\right)$,

(d) $\alpha_{1}, \alpha_{2} \in W^{1, \infty}(Y)$

(e) $K \in L^{\infty}\left(V \times \overline{\mathbb{B}}_{s_{2}}(0)\right)$ is $\lambda$-Lipschitz continuous w.r.t. its second argument for some $\lambda>0$,

(f) $p_{c}, p_{h} \geq 0, \psi \geq 0$ and $\alpha_{1}, \alpha_{2} \geq 0, K \geq 0$.

\section{Proposition 3.6 (Existence and uniqueness of weak solutions)}

Under model assumptions $(a)$ - (e), the initial value problem (23) has a unique weak solution $\left.\left.f:[0, T] \longrightarrow L^{2}(Z), Q:[0, T] \longrightarrow L^{2}\left(\mathbb{R}^{n} \times \mathbb{S}^{n-1}\right), L:\right] 0, T\right] \longrightarrow W^{1,2}\left(\mathbb{R}^{n}\right)$ for each tuple of initial states $f_{0} \in L^{2}(Z), Q_{0} \in L^{2}\left(\mathbb{R}^{n} \times \mathbb{S}^{n-1}\right), L_{0} \in L^{2}\left(\mathbb{R}^{n}\right)$.

\section{Corollary 3.7 (Nonnegativity of weak solutions)}

In addition to assumptions $(a)-(e)$ suppose property $(f)$ now. Then the initial sign conditions $f_{0} \geq 0, Q_{0} \geq 0, L_{0} \geq 0$ imply $f(t) \geq 0, Q(t) \geq 0$ and $L(t) \geq 0$ for every $t \in[0, T]$.

This corollary is proved in detail in Section 7.5 below. Hence Proposition 3.6 remains to be verified as a consequence of Corollary 3.4 .

Indeed, assumptions (i) - (iii) of Theorem 3.2 are obviously satisfied. In regard to assumption (iv) about local Lipschitz continuity, Definition 3.1 implies

$$
\left|\int_{Z} \varphi \cdot\left(f_{1}-f_{2}\right) d \mathbf{z}\right| \leq d^{f}\left(f_{1}, f_{2}\right) \cdot \max \left\{\|\varphi\|_{L^{2}},\|\varphi\|_{L^{\infty}},\left\|\nabla_{\mathbf{y}} \varphi\right\|_{L^{\infty}}\right\}
$$

for all $f_{1}, f_{2} \in L^{2}(Z)$ and every test function $\varphi \in C_{c}^{0}(Z)$ with $\nabla_{\mathbf{y}} \varphi \in L^{\infty}$. This estimate holds in particular whenever $\varphi$ does not depend on $\mathbf{y}$ explicitly and so, it is easy to conclude from 
Hölder inequality and $C_{c}^{\infty}(Z)$ being dense in $L^{2}(Z)$

$$
\begin{aligned}
\left\|\mathcal{R}\left(t, f_{1}, Q, L\right)-\mathcal{R}\left(t, f_{2}, Q, L\right)\right\|_{L^{2}(Z)} & \leq \operatorname{const}\left(\gamma_{Q}, r_{\mathrm{ECM}}, R_{0}, V\right) \cdot d^{f}\left(f_{1}, f_{2}\right), \\
\left\|\mathcal{R}\left(t, f, Q_{1}, L\right)-\mathcal{R}\left(t, f, Q_{2}, L\right)\right\|_{L^{2}(Z)} & \leq \operatorname{const}\left(\gamma_{Q}, r_{\mathrm{ECM}}, R_{0}, V,\|f\|_{L^{2}(Z)}\right) \cdot\left\|Q_{1}-Q_{2}\right\|_{L^{2}}, \\
\left\|\mathcal{R}\left(t, f, Q, L_{1}\right)-\mathcal{R}\left(t, f, Q, L_{2}\right)\right\|_{L^{2}(Z)} & \leq r_{L} \cdot\left\|L_{1}-L_{2}\right\|_{L^{2}}
\end{aligned}
$$

for all $f, f_{1}, f_{2} \in L^{2}(Z), Q, Q_{1}, Q_{2} \in L^{2}\left(\mathbb{R}^{n} \times \mathbb{S}^{n-1}\right)$ and $L, L_{1}, L_{2} \in W^{1,2}\left(\mathbb{R}^{n}\right)$. The corresponding conclusions about Lipschitz continuity can also be drawn for the coefficient functions $\mathcal{G}, \mathcal{U}, \mathcal{T}, \mathcal{W}$. In connection with $\mathcal{W}(t, f, Q, \cdot)$, in particular, it proves to be helpful that the projection on any compact convex set (such as $\overline{\mathrm{co}} V=\overline{\mathbb{B}}_{s_{2}}(0)$ here) is Lipschitz continuous. Assumption (b) about $p_{c}, p_{h}$ implies that all coefficients $\mathcal{W}(t, f, Q, L) \in L^{2}(Z)$ have compact support and so, condition (v) in main Theorem 3.2 is obviously satisfied. Similarly assumption (f) ensures condition (vii) in Corollary 3.5 (about preserving nonnegativity).

Finally condition (vi) in Corollary 3.4 remains to be verified. The preceding choice of $\mathcal{G}$ and $Y \stackrel{\text { Def. }}{=}\left\{\left(y_{1}, y_{2}\right) \in\right] 0, R_{0}\left[^{2} \mid y_{1}+y_{2}<R_{0}\right\}$ even leads to

$$
\mathbf{y}+h \cdot \mathcal{G}(t, f, Q, L)(\mathbf{x}, \mathbf{v}, \mathbf{y}) \in \bar{Y}
$$

for every $\mathbf{y} \in \partial Y$ and $h>0$ sufficiently small as well as all $t, f, Q, L, \mathbf{x}, \mathbf{v}$. 


\section{The conceptual strategy how to prove main Theorem 3.2}

\subsection{The method of successive approximation for the whole system}

The proof of main Theorem 3.2 combines two well-known strategies: First the basic concept is the method of successive approximation. Indeed, for any appropriately given function triple $(\widetilde{f}, \widetilde{Q}, \widetilde{L})$ of time, there exist $f:[0, T] \longrightarrow L^{2}(Z), Q:[0, T] \longrightarrow L^{2}\left(\mathbb{R}^{n} \times \mathbb{S}^{n-1}\right)$ and $\left.\left.L:\right] 0, T\right] \longrightarrow$ $W^{1,2}\left(\mathbb{R}^{n}\right)$ which form the unique weak solutions of nonautonomous, but (semi-) linear initial value problem

$$
\left\{\begin{aligned}
\partial_{t} f+\operatorname{div}_{\mathbf{x}}(f \mathbf{v})+\operatorname{div}_{\mathbf{y}}(f \mathcal{G}(t, \widetilde{f}, \widetilde{Q}, \widetilde{L})) & =\mathcal{U}(t, \widetilde{f}, \widetilde{Q}, \widetilde{L}) f+\mathcal{W}(t, \widetilde{f}, \widetilde{Q}, \widetilde{L}), & & f(0)=f_{0}, \\
\partial_{t} Q & =\mathcal{T}(t, \widetilde{f}, \widetilde{Q}, \widetilde{L}), & & Q(0)=Q_{0}, \\
\partial_{t} L & =\kappa_{L} \Delta_{\mathbf{x}} L+\mathcal{R}(t, \widetilde{f}, \widetilde{Q}, L), & & L(0)=L_{0} .
\end{aligned}\right.
$$

The related map $\mathcal{L}:(\widetilde{f}, \widetilde{Q}, \widetilde{L}) \longmapsto(f, Q, L)$ proves to be a strict contraction for some sufficiently small time interval $[0, \tau]$ and so, Banach's fixed point theorem provides a unique solution to the full system in $[0, \tau]$.

Second this local existence can be extended to any finite time interval $[0, T]$ iteratively since the duration $\tau>0$ has a positive lower bound depending only on norms of the given initial values $f_{0}, L_{0}, Q_{0}$ and the coefficient functions.

The combination of these two notions is standard and, more details about this special system are presented in Section 7. Sections 5 and 6.1, 6.2 in between focus on the three nonautonomous (semi-) linear equations of the system separately. Each time we investigate the existence of unique solutions and their continuous dependence on the coefficients. The latter implies their Lipschitz continuous dependence on the given data $\widetilde{f}, \widetilde{Q}, \widetilde{L}$.

\subsection{The linear subproblem of $Q(\cdot)$}

The subproblem of $Q(\cdot)$

$$
\left\{\begin{aligned}
\partial_{t} Q & =\mathcal{T}(t, \widetilde{f}(t), \widetilde{Q}(t), \widetilde{L}(t)) \\
Q(0) & =Q_{0}
\end{aligned}\right.
$$

has formal similarities with an ordinary differential equation. But it is even simpler because the wanted function $Q(\cdot)$ does not occur on the right-hand side. Hence we need just an appropriate form of integration w.r.t. time. The solution $Q(\cdot)$ is a function of time whose values are in the Banach space $L^{2}\left(\mathbb{R}^{n} \times \mathbb{S}^{n-1}\right)$ and, this observation motivates Bochner integrals.

Furthermore weak solutions of these problems are shown to be unique and, so we dispense with the search for any other solutions (but the Bochner integrals just mentioned). The details are collected in Section 6.1. 


\subsection{The semilinear subproblem of $L(\cdot)$}

$L(\cdot)$ is characterised in terms of a nonautonomous semilinear reaction-diffusion equation

$$
\left\{\begin{aligned}
\partial_{t} L & =\kappa_{L} \Delta_{\mathbf{x}} L+\mathcal{R}(t, \widetilde{f}(t), \widetilde{Q}(t), L(t)) \\
L(0) & =L_{0}
\end{aligned}\right.
$$

Its solution exists since the reaction term $\mathcal{R}$ is assumed to be locally Lipschitz continuous w.r.t. $L$ and of linear growth w.r.t. $L$. For further estimates, it can be formulated implicitly by means of the scaled fundamental solution since we consider the problem in the whole space $\mathbb{R}^{n}$ (see Proposition 6.5 below).

This type of parabolic differential equation is known to have some instantaneously smoothing effect on the solution, i.e. here: for any $L_{0} \in L^{2}\left(\mathbb{R}^{n}\right)$, the solution $L(\cdot)$ satisfies $L(t) \in W^{1,2}\left(\mathbb{R}^{n}\right)$ (at least) at every time $t>0$. This general feature opens the door to formulating some assumptions w.r.t. the Sobolev norm $\|\cdot\|_{W^{1,2}\left(\mathbb{R}^{n}\right)}$ instead of the $L^{2}$ norm. In main Theorem 3.2, we use this aspect in assumption (iv) about the Lipschitz continuity of the coefficient functions $\mathcal{G}, \mathcal{U}, \mathcal{W}, \mathcal{T}$, for example.

The price to pay for these weaker continuity hypotheses is that we need a priori bounds of the gradient $\nabla_{\mathbf{x}} L(t)$ - particularly for verifying the strict contraction of the successive approximation map $\mathcal{L}$. The proof of Proposition 6.5 reveals such estimates which are collected in Corollary 6.6. Roughly speaking, we obtain $\left\|\nabla_{\mathbf{x}} L(t)\right\|_{L^{2}\left(\mathbb{R}^{n}\right)} \leq$ const $\cdot\left(1+t^{-1 / 2}\right)$ which is mainly relevant for small $t>0$.

\subsection{The linear subproblem of $f(\cdot)$}

The nonautonomous linear problem for component $f(\cdot)$ is probably the most challenging subproblem - if we avoid generous assumptions about the regularity of its coefficients:

$$
\left\{\begin{aligned}
\partial_{t} f+\operatorname{div}_{\mathbf{x}}(f \mathbf{v})+\operatorname{div}_{\mathbf{y}}(f \widetilde{\mathbf{g}}(t)) & =\widetilde{u}(t) f+\widetilde{w}(t) \text { in }[0, T], \\
f(0) & =f_{0}
\end{aligned}\right.
$$

just with $\quad \widetilde{\mathbf{g}}(t):=\mathcal{G}(t, \widetilde{f}(t), \widetilde{Q}(t), \widetilde{L}(t)) \in L^{2}\left(Z, \mathbb{R}^{d}\right) \cap L^{\infty}\left(Z, \mathbb{R}^{d}\right), \quad \partial_{\mathbf{y}} \widetilde{\mathbf{g}}(t) \in L^{\infty}\left(Z, \mathbb{R}^{d \times d}\right)$,

$$
\begin{aligned}
\widetilde{u}(t) & :=\mathcal{U}(t, \widetilde{f}(t), \widetilde{Q}(t), \widetilde{L}(t)) \in L^{2}(Z) \quad \cap L^{\infty}(Z), \quad \nabla_{\mathbf{y}} \widetilde{u}(t) \in L^{\infty}\left(Z, \mathbb{R}^{d}\right), \\
\widetilde{w}(t) & :=\mathcal{W}(t, \widetilde{f}(t), \widetilde{Q}(t), \widetilde{L}(t)) \in L^{2}(Z) .
\end{aligned}
$$

This is a nonautonomous linear transport equation and, we are interested in weak solutions $f(\cdot)$ with values in $L^{2}(Z)$.

In general, the existence of weak solutions is not so difficult to obtain by means of smoothing coefficients. But here the transport equation is just part of system (24) preparing the full nonlinear problem (20). Thus we need the continuous dependence of the solution on the timedependent coefficients $\widetilde{\mathbf{g}}, \widetilde{u}, \widetilde{w}$. It should be even provided in form of an explicit inequality that we can then use for verifying the strictly contractive feature of the successive approximation map $\mathcal{L}$.

This is closely related to the uniqueness of weak solutions. If the coefficients are not sufficiently regular for the standard method of characteristics then this latter topic belongs to the current 
fields of research (see, e.g., [1, 2, 3, 11, 45, 47] and references therein).

The subsequent investigation of the nonautonomous linear subproblem (25) is motivated by similar results about signed Radon measures on $\mathbb{R}^{n}$ in $[41, \S 2.5]$ and, it consists of the following steps presented in Section 5:

1. The choice of metrics on $L^{2}(Z) \stackrel{\text { Def. }}{=} L^{2}\left(\mathbb{R}^{n} \times V \times Y\right)$

The state space $L^{2}(Z)$ of cell densities is not supplied with the $L^{2}$ norm directly. We prefer the metric $d^{f}: L^{2}(Z) \times L^{2}(Z) \longrightarrow[0, \infty[$ instead - for two reasons:

$$
\begin{array}{r}
d^{f}(f, g):=\sup \left\{\int_{Z} \varphi \cdot(f-g) d \mathbf{z} \mid \varphi \in C_{c}^{0}(Z), \quad \nabla_{\mathbf{y}} \varphi \in L^{\infty}(Z),\right. \\
\left.\|\varphi\|_{L^{2}} \leq 1,\|\varphi\|_{L^{\infty}} \leq 1,\left\|\nabla_{\mathbf{y}} \varphi\right\|_{L^{\infty}} \leq 1\right\}
\end{array}
$$

First, but of rather minor priority, it proves to be useful in the subsequent estimates comparing weak solutions because we can represent them in terms of characteristic flows whose compositions imply invariance of the class of test functions $\varphi$.

From our point of view, the second reason is more relevant for modelling biological systems. The metric $d^{f}$ reflects the comparison of "features" - in the sense of linear forms. Indeed, every linear form relates the cell density $f \in L^{2}(Z)$ to a real number and, it can be represented by means of some function $\varphi \in L^{2}(Z)$ :

$$
L^{2}(Z) \longrightarrow \mathbb{R}, \quad f \longmapsto \int_{Z} \varphi \cdot f d \mathbf{z}
$$

This real-valued "feature" might hardly change when considering two functions $f, g \in$ $L^{2}(Z)$ although the $L^{2}$ norm $\|f-g\|_{L^{2}(Z)}$ is rather large. In general, it is well known

$$
\|f-g\|_{L^{2}(Z)}=\sup \left\{\int_{Z} \varphi \cdot(f-g) d \mathbf{z} \mid \varphi \in L^{2}(Z),\|\varphi\|_{L^{2}} \leq 1\right\}
$$

(see, e.g., [64, § IV.9]). The metric $d^{f}$, however, considers the supremum w.r.t. a smaller class of test functions $\varphi$, i.e., we focus on a selection of "features" which, in particular, restrict the oscillation w.r.t. $\mathbf{y}:\left\|\nabla_{\mathbf{y}} \varphi\right\|_{L^{\infty}} \leq 1$. Suggesting a mathematically suitable class of test functions for the metric is part of our contribution to this model problem.

The metric $d^{f}$ concerns mainly the continuous dependence of solutions on initial values and coefficients (as summarised in estimates (7.), (8.) of Proposition 5.21). For their (Lipschitz) continuity w.r.t. time, we prefer the modified metrics

$$
\begin{aligned}
& \breve{e}^{f}:(f, g) \longmapsto \sup \left\{\int_{Z} \varphi \cdot(f-g) d \mathbf{z} \mid \varphi \in C_{c}^{1}(Z),\|\varphi\|_{W^{1,2}} \leq 1,\|\varphi\|_{W^{1, \infty}} \leq 1\right\} \\
& e^{f}:(f, g) \longmapsto \breve{e}^{f}(f, g)+\left|\|f\|_{L^{2}(Z)}-\|g\|_{L^{2}(Z)}\right|
\end{aligned}
$$

$\breve{e}^{f}$ differs from $d^{f}$ in the class of test functions $\varphi$ whereas $e^{f}$ takes additionally the deviation of $L^{2}$ norms into consideration. 
In Section 5.1, we investigate the relation between the metrics $d^{f}, e^{f}$ and more popular topologies of $L^{2}(Z)$. Under some additional assumptions like tightness, $\breve{e}^{f}$ proves to metrize the weak topology and, $e^{f}$ induces the norm topology of $L^{2}(Z)$ (see Lemma 5.3 and Proposition 5.5). The latter proves to be equivalent to the combination of $d^{f}$ and the convergence of $L^{2}$ norms.

As a consequence of these relations to weak and norm topology, every tight and weakly closed subset of $L^{2}(Z)$ is complete w.r.t. the metric $d^{f}$ (see Lemma 5.6) and, that is actually what we need for constructing solutions approximatively.

\section{Nonautonomous linear problem: Uniqueness of weak solutions}

Here we cannot apply the concept of "renormalised solutions" introduced by DiPerna and Lions [19] immediately since $\widetilde{\mathbf{g}}$ is assumed to have weak partial derivatives w.r.t. $\mathbf{y}$ only, but we can still adapt the arguments of Le Bris and Lions [40] to square integrable weak solutions.

3. Autonomous linear problem with "more regular" coefficients: Existence of solutions

We start with the autonomous linear problem under the assumptions $\mathbf{g} \in C_{c}^{1}\left(Z, \mathbb{R}^{d}\right)$, $u \in C^{1}(Z) \cap W^{1, \infty}(Z)$. This is much "more regular" than the hypotheses of Theorem 3.2 , but it has the technical advantage that the notion of characteristics leads to a representation of $\int_{Z} \varphi f(t) d \mathbf{z}$ for the solution $f:[0, T] \longrightarrow L^{2}(Z)$ and any test function $\varphi \in C_{c}^{1}(Z)$ (see Proposition 5.11 including an a priori bound of $\|f(t)\|_{L^{2}(Z)}$ ).

4. Autonomous linear problem with "more regular" coefficients: An explicit formula

The presented solution $f:[0, T] \longrightarrow L^{2}(Z)$ can even be described explicitly - simply by means of the theorems of Fubini and transformations. This formula presented in Proposition 5.18, however, does not provide any significant technical advantages for subsequent estimates. Its main purpose is the upper bound mentioned in Corollary 5.19:

$$
|f(t, \xi)| \leq e^{\left(\left\|\left[\operatorname{div}_{\mathbf{y}} \mathbf{g}\right]^{-}\right\|_{L} \infty+\|u\|_{L} \infty\right) \cdot t} \cdot\left(\left|f_{0}\left(\mathfrak{Z}_{-1, \mathbf{g}}(t ; \xi)\right)\right|+\int_{0}^{t}\left|w\left(\mathfrak{Z}_{-1, \mathbf{g}}(t-s ; \xi)\right)\right| d s\right) .
$$

The function on the right-hand side will help us later on to verify tightness of all solutions while perturbing coefficients in a suitably bounded way.

5. Autonomous linear problem with "more regular" coefficients: Further estimates

Still considering the stronger assumptions $\mathbf{g} \in C_{c}^{1}\left(Z, \mathbb{R}^{d}\right), u \in C^{1}(Z) \cap W^{1, \infty}(Z)$ and $w \in L^{2}(Z)$, we then investigate the function

$$
\vartheta_{\mathbf{g}, u, w}^{f}: \quad[0,1] \times L^{2}(Z) \longrightarrow L^{2}(Z), \quad\left(t, f_{0}\right) \longmapsto f(t)
$$


induced by the unique weak solution of

$$
\left\{\begin{aligned}
\partial_{t} f+\operatorname{div}_{\mathbf{x}}(f \mathbf{v})+\operatorname{div}_{\mathbf{y}}(f \mathbf{g}) & =u f+w \text { in }[0, t], \\
f(0) & =f_{0} .
\end{aligned}\right.
$$

$\vartheta_{\mathbf{g}, u, w}^{f}$ has the typical properties of a semi-dynamical system on $L^{2}(Z)$. It is $\omega$-contractive w.r.t. $d^{f}$ and, the related constant $\omega$ can be estimated explicitly in terms of $n,\left\|\operatorname{div}_{\mathbf{y}} \mathbf{g}\right\|_{L^{\infty}}$, $\|u\|_{L^{\infty}},\left\|\nabla_{\mathbf{y}} u\right\|_{L^{\infty}}$.

Furthermore $\vartheta_{\mathbf{g}, u, w}^{f}\left(\cdot, f_{0}\right):[0,1] \longrightarrow L^{2}(Z)$ is Lipschitz continuous w.r.t. $e^{f}$ with

$$
\operatorname{Lip} \vartheta_{\mathbf{g}, u, w}^{f}\left(\cdot, f_{0}\right) \leq \operatorname{const}\left(n,\|\mathbf{g}\|_{L^{2}},\left\|\operatorname{div}_{\mathbf{y}} \mathbf{g}\right\|_{L^{\infty}},\|u\|_{L^{\infty}}, V\right) \cdot\left(\left\|f_{0}\right\|_{L^{2}}+\|w\|_{L^{2}}\right),
$$

i.e., the Lipschitz continuity is locally uniform w.r.t. the initial function $f_{0}$. Last, but not least, we verify that in the metric space $\left(L^{2}(Z), d^{f}\right)$, the function $\vartheta_{\mathbf{g}, u, w}^{f}\left(t, f_{0}\right)$ depends on the coefficients $\mathbf{g}, u, w$ in a Lipschitz continuous way. In this context, the autonomous coefficients $\mathbf{g}, u, w$ are supplied with the $L^{2}$ norm (see Proposition 5.21). This choice lays the foundations for the next step:

6. Extending existence and estimates for autonomous linear problems to "less regular" coefficients: $\mathbf{g} \in L^{\infty}\left(Z, \mathbb{R}^{d}\right) \cap L^{2}, \partial_{\mathbf{y}} \mathbf{g} \in L^{\infty}, u \in L^{\infty}(Z) \cap L^{2}, \nabla_{\mathbf{y}} u \in L^{\infty}\left(Z, \mathbb{R}^{d}\right), w \in L^{2}(Z)$ Now the coefficients have the same regularity as in Theorem 3.2, but are still autonomous. The corresponding solution map $\vartheta_{\mathbf{g}, u, w}^{f}: \quad[0,1] \times L^{2}(Z) \longrightarrow L^{2}(Z)$ is constructed by smoothing the coefficients $\mathbf{g}, u$ such that the preceding estimates can be applied.

In particular, the solutions of the smoothed problems are tight and form a uniform Cauchy sequence w.r.t. $d^{f}$. Thus a limit function exists and it is the wanted weak solution related to the "less regular" coefficients $\mathbf{g}, u, w$. (Its uniqueness was proven before.)

This approach has the advantage that all our a priori estimates (in regard to continuous dependence on data, for example) are preserved. Hence $\vartheta_{\mathbf{g}, u, w}^{f}$ has still the typical features of a semi-dynamical system on $L^{2}(Z)$ which is $\omega$-contractive w.r.t. $d^{f}$ and Lipschitz continuous in time w.r.t. $e^{f}$.

In the terminology of $[41, \S 3]$, this map $\vartheta_{\mathbf{g}, u, w}:[0,1] \times L^{2}(Z) \longrightarrow L^{2}(Z)$ is an example for a "transition" on the tuple $\left(L^{2}(Z), d^{f}, e^{f},\|\cdot\|_{L^{2}(Z)}\right)$. For the sake of a self-contained presentation, we do not draw any conclusions from the general (but slightly abstract) results in $[41, \S 3.3 .7]$, but give all further proofs explicitly here.

7. Nonautonomous linear problem with "less regular" coefficients:

Existence of weak solutions and a priori bounds

The preceding step serves a rather preparatory purpose. It clarifies how smoothing coefficients, tightness of the related solutions and their continuous dependence on data lead to the wanted solution via a limit process. Essentially the same arguments are applied 
in Proposition 5.26 (more briefly) for solving the nonautonomous linear problem

$$
\left\{\begin{aligned}
\partial_{t} f+\operatorname{div}_{\mathbf{x}}(f \mathbf{v})+\operatorname{div}_{\mathbf{y}}(f \widetilde{\mathbf{g}}(t)) & =\widetilde{u}(t) f+\widetilde{w}(t) \quad \text { in }[0, T], \\
f(0) & =f_{0}
\end{aligned}\right.
$$

with coefficients $\widetilde{\mathbf{g}} \in L^{1}\left(0, T ; L^{2}\left(Z, \mathbb{R}^{d}\right)\right), \widetilde{u} \in L^{1}\left(0, T ; L^{2}(Z)\right), \widetilde{w} \in L^{\infty}\left(0, T ; L^{2}(Z)\right)$ of appropriate regularity for main Theorem 3.2.

\section{The subproblem for cell concentration $f$ on $Z=\mathbb{R}^{n} \times V \times \mathbb{R}^{d}$}

\subsection{The metrics $d^{f}, e^{f}$ of the state space $L^{2}(Z)$}

Definition 5.1 In addition to the metric $d^{f}: L^{2}(Z) \times L^{2}(Z) \longrightarrow[0, \infty[$ in Definition 3.1,

$$
\begin{aligned}
d^{f}(f, g):=\sup \left\{\int_{Z} \varphi \cdot(f-g) d \mathbf{z} \mid \varphi \in C_{c}^{0}(Z), \quad \nabla_{\mathbf{y}} \varphi \in L^{\infty}(Z),\right. \\
\left.\|\varphi\|_{L^{2}} \leq 1,\|\varphi\|_{L^{\infty}} \leq 1,\left\|\nabla_{\mathbf{y}} \varphi\right\|_{L^{\infty}} \leq 1\right\}
\end{aligned}
$$

set

$$
\begin{aligned}
\breve{e}^{f}: L^{2}(Z) \times L^{2}(Z) & \longrightarrow[0, \infty[ \\
(f, g) & \longmapsto \sup \left\{\int_{Z} \varphi \cdot(f-g) d \mathbf{z} \mid \varphi \in C_{c}^{1}(Z),\|\varphi\|_{W^{1,2}} \leq 1,\|\varphi\|_{W^{1, \infty}} \leq 1\right\} \\
e^{f}: L^{2}(Z) \times L^{2}(Z) & \longrightarrow[0, \infty[ \\
(f, g) & \longmapsto \breve{e}^{f}(f, g)+\left|\|f\|_{L^{2}(Z)}-\|g\|_{L^{2}(Z)}\right| .
\end{aligned}
$$

Remark 5.2 (1.) Obviously the following inequalities hold for all $f, g \in L^{2}(Z)$ :

$$
\breve{e}^{f}(f, g) \leq d^{f}(f, g) \leq\|f-g\|_{L^{2}(Z)}, \quad e^{f}(f, g) \leq 2 \cdot\|f-g\|_{L^{2}(Z)} .
$$

(2.) The basic notion of $\breve{e}^{f}$ is quite similar to the "bounded Lipschitz distance" of Radon measures a.k.a. Fortet-Mourier distance [63] a.k.a. $W^{1, \infty}$ dual metric [41]. It differs in two regards though: First the smooth test functions are supposed to have compact support. Second they have to satisfy an upper bound on the $W^{1,2}$ norm.

Lemma 5.3 $\breve{e}^{f}$ metrizes the weak topology on norm-bounded tight balls in $L^{2}(Z)-$ in the following sense: Suppose $f \in L^{2}(Z)$ and let $\left(f_{k}\right)_{k \in \mathbb{N}}$ be any sequence in $L^{2}(Z)$ such that $\left(\left|f_{k}\right|^{2}\right)$ is tight in $Z$, i.e. $\lim _{\rho \rightarrow \infty} \sup _{k \in \mathbb{N}}\left\|f_{k}\right\|_{L^{2}\left(Z \backslash\left(\mathbb{B}_{\rho}(0) \times V \times \mathbb{B}_{\rho}(0)\right)\right)}=0$. Then,

$$
f_{k} \longrightarrow f \text { weakly in } L^{2}(Z)(k \longrightarrow \infty) \Longleftrightarrow\left\{\begin{array}{l}
\sup _{k \in \mathbb{N}}\left\|f_{k}\right\|_{L^{2}(Z)}<\infty \text { and } \\
\lim _{k \rightarrow \infty} \breve{e}^{f}\left(f_{k}, f\right)=0 .
\end{array}\right.
$$


Proof. " $\Longleftarrow " \quad$ This is an immediate consequence of $C_{c}^{1}(Z)$ being a dense subset of $L^{2}(Z)=\left(L^{2}(Z)\right)^{*}$ (see, e.g., $[64$, V.1.Theorem 3]).

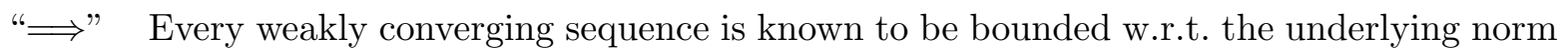
as a consequence of the Banach-Steinhaus theorem. Set $S:=\sup _{k \in \mathbb{N}}\left\|f_{k}\right\|_{L^{2}(Z)}<\infty$. Due to the lower semicontinuity of the norm w.r.t. weak convergence, we have $\|f\|_{L^{2}(Z)} \leq S$.

For proving $\breve{e}^{f}\left(f_{k}, f\right) \longrightarrow 0$, choose $\varepsilon>0$ arbitrarily. There is a radius $\rho \geq 1$ with

$$
\|f\|_{L^{2}\left(Z \backslash\left(\mathbb{B}_{\rho}(0) \times V \times \mathbb{B}_{\rho}(0)\right)\right)}+\sup _{k \in \mathbb{N}}\left\|f_{k}\right\|_{L^{2}\left(Z \backslash\left(\mathbb{B}_{\rho}(0) \times V \times \mathbb{B}_{\rho}(0)\right)\right)}<\frac{\varepsilon}{2}
$$

since $\left(\left|f_{k}\right|^{2}\right)_{k \in \mathbb{N}}$ is tight by assumption. According to the Sobolev embedding theorem, the set $\left\{\varphi \in C_{c}^{1}\left(\mathbb{B}_{2 \rho}(0) \times V \times \mathbb{B}_{2 \rho}(0)\right) \mid\|\varphi\|_{W^{1,2}},\|\varphi\|_{W^{1, \infty}} \leq 1\right\}$ (i.e., their zero extensions to $Z$, strictly speaking) is relatively compact in $\left(L^{2}(Z),\|\cdot\|_{L^{2}(Z)}\right)$. Hence there exist finitely many functions $\varphi_{1} \ldots \varphi_{j} \in C_{c}^{1}\left(\mathbb{B}_{2 \rho}(0) \times V \times \mathbb{B}_{2 \rho}(0)\right)($ with $j=j(\varepsilon, \rho) \in \mathbb{N})$ s.t. $\sup _{i \leq j}\left\{\left\|\varphi_{i}\right\|_{W^{1,2}},\left\|\varphi_{i}\right\|_{W^{1, \infty}}\right\} \leq 1$,

$$
\left\{\varphi \in C_{c}^{1}\left(\mathbb{B}_{2 \rho} \times V \times \mathbb{B}_{2 \rho}\right) \mid\|\varphi\|_{W^{1,2}},\|\varphi\|_{W^{1, \infty}} \leq 1\right\} \subseteq \bigcup_{i=1}^{j}\left\{g \in L^{2}(Z) \mid\left\|g-\varphi_{i}\right\|_{L^{2}}<\frac{\varepsilon}{4 S+1}\right\} .
$$

Then we obtain

$$
\begin{aligned}
\breve{e} f\left(f_{k}, f\right) & \stackrel{\text { Def. }}{=} \sup \left\{\int_{Z} \varphi \cdot\left(f_{k}-f\right) d \mathbf{z} \mid \varphi \in C_{c}^{1}(Z),\|\varphi\|_{W^{1,2}} \leq 1,\|\varphi\|_{W^{1, \infty}} \leq 1\right\} \\
& \leq \sup \left\{\int_{Z} \varphi \cdot\left(f_{k}-f\right) d \mathbf{z} \mid \varphi \in C_{c}^{1}\left(\mathbb{B}_{2 \rho} \times V \times \mathbb{B}_{2 \rho}\right),\|\varphi\|_{W^{1,2}},\|\varphi\|_{W^{1, \infty}} \leq 1\right\}+\frac{\varepsilon}{2} \\
& \leq \sup _{1 \leq i \leq j} \int_{Z} \varphi_{i} \cdot\left(f_{k}-f\right) d \mathbf{z}+\frac{\varepsilon}{4 S+1}\left\|f_{k}-f\right\|_{L^{2}(Z)} \\
& \leq \sup _{1 \leq i \leq j} \int_{Z} \varphi_{i} \cdot\left(f_{k}-f\right) d \mathbf{z}+\varepsilon
\end{aligned}
$$

i.e. $\quad \limsup _{k \rightarrow \infty} \breve{e}^{f}\left(f_{k}, f\right)=0$.

\section{Corollary 5.4}

Every norm-bounded closed tight subset of $L^{2}(Z)$ is (sequentially) compact w.r.t. $\breve{e}^{f}$.

Proof. The Hilbert space $L^{2}(Z)$ is reflexive and so, every bounded closed ball in $L^{2}(Z)$ is known to be sequentially compact with respect to the weak topology (e.g.,[64, V.1.Theorem 1]). The equivalence in Lemma 5.3 then implies convergence w.r.t. $\breve{e}^{f}$.

Proposition 5.5 The following equivalence holds for any sequence $\left(f_{k}\right)_{k \in \mathbb{N}}$ in $L^{2}(Z)$ :

$$
\left\|f_{k}-f\right\|_{L^{2}(Z)} \stackrel{k \rightarrow \infty}{\longrightarrow} 0 \Longleftrightarrow\left\{\begin{array} { l } 
{ \operatorname { l i m } _ { k \rightarrow \infty } e ^ { f } ( f _ { k } , f ) = 0 } \\
{ ( | f _ { k } | ^ { 2 } ) _ { k \in \mathbb { N } } \text { is tight in } Z . }
\end{array} \Longleftrightarrow \left\{\begin{array}{l}
\lim _{k \rightarrow \infty}\left\|f_{k}\right\|_{L^{2}(Z)}=\|f\|_{L^{2}} \\
\lim _{k \rightarrow \infty} d^{f}\left(f_{k}, f\right)=0 \\
\left(\left|f_{k}\right|^{2}\right)_{k \in \mathbb{N}} \text { is tight in } Z .
\end{array}\right.\right.
$$


Proof. It results essentially from $L^{2}(Z)$ being a Hilbert space. Due to [64, V.1.Theorem 8], every weakly converging sequence $\left(f_{k}\right)_{k \in \mathbb{N}}$ in the Hilbert space $L^{2}(Z)$ has the property

$$
\left\|f_{k}-f\right\|_{L^{2}(Z)} \longrightarrow 0(k \longrightarrow \infty) \Longleftrightarrow\left\|f_{k}\right\|_{L^{2}(Z)} \longrightarrow\|f\|_{L^{2}(Z)}(k \longrightarrow \infty) .
$$

Hence the first claimed equivalence is a direct consequence of Lemma 5.3. Finally the second equivalence results from Remark 5.2 (1.).

Lemma 5.6 Norm-bounded closed convex tight subsets of $L^{2}(Z)$ are complete with respect to $d^{f}$ - in the following sense: Let $M \subseteq L^{2}(Z)$ be any norm-bounded closed convex subset with $\lim _{\rho \rightarrow \infty} \sup _{f \in M}\|f\|_{L^{2}\left(Z \backslash\left(\mathbb{B}_{\rho}(0) \times V \times \mathbb{B}_{\rho}(0)\right)\right)}=0$.

Then every Cauchy sequence w.r.t. $d^{f}$ in $M$ has a limit in $M$ w.r.t. $d^{f}$.

Proof. Let $\left(f_{k}\right)_{k \in \mathbb{N}}$ be a Cauchy sequence in $M$ w.r.t. $d^{f}$. Then $\left(f_{k}\right)_{k \in \mathbb{N}}$ is Cauchy sequence w.r.t. $\breve{e}^{f}$ due to Remark 5.2 (1.). Hence Corollary 5.4 provides a function $f \in L^{2}(Z)$ with $\breve{e}^{f}\left(f_{k}, f\right) \longrightarrow 0(k \longrightarrow \infty)$ or equivalently $f_{k} \longrightarrow f$ weakly in $L^{2}(Z)$.

As a consequence of Mazur's Lemma (see, e.g., [64, V.1.Theorem 2]), the norm-closed convex set $M \subseteq L^{2}(Z)$ is weakly closed and so $f \in M$. It remains to prove $d^{f}\left(f_{k}, f\right) \longrightarrow 0$ for $k \longrightarrow \infty$.

Choose any $\varepsilon>0$. As $\left(f_{k}\right)_{k \in \mathbb{N}}$ is Cauchy sequence w.r.t. $d^{f}$, there is some $J=J(\varepsilon) \in \mathbb{N}$ with

$$
d^{f}\left(f_{k}, f_{l}\right) \leq \varepsilon \quad \text { for all } k, l \geq J .
$$

For every test function $\varphi \in C_{c}^{0}(Z)$ with $\|\varphi\|_{L^{2}} \leq 1,\|\varphi\|_{L^{\infty}} \leq 1,\left\|\nabla_{\mathbf{y}} \varphi\right\|_{L^{\infty}} \leq 1$ and any index $k \geq J$, we conclude from the weak convergence of $\left(f_{l}\right)_{l \in \mathbb{N}}$ to $f$

$$
\begin{aligned}
\int_{Z} \varphi \cdot\left(f_{k}-f\right) d \mathbf{z} & =\limsup _{l \rightarrow \infty} \int_{Z} \varphi \cdot\left(f_{k}-f_{l}\right) d \mathbf{z} \\
& \leq \limsup _{l \rightarrow \infty} d^{f}\left(f_{k}, f_{l}\right) \leq \varepsilon
\end{aligned}
$$

i.e. $\quad d^{f}\left(f_{k}, f\right) \leq \varepsilon$ holds for every index $k \geq J$. 


\subsection{Nonautonomous linear problem: Uniqueness of weak solutions}

Proposition 5.7 For any $T>0$ suppose $\widetilde{\mathbf{g}} \in L^{\infty}\left(0, T ; L^{\infty}\left(Z, \mathbb{R}^{d}\right)\right), \partial_{\mathbf{y}} \widetilde{\mathbf{g}} \in L^{\infty}(0, T$; $\left.L^{\infty}\left(Z, \mathbb{R}^{d \times d}\right)\right), \widetilde{u} \in L^{\infty}\left(0, T ; L^{\infty}(Z)\right)$ and $\widetilde{w} \in L^{1}\left(0, T ; L^{2}(Z)\right)$.

Then for any initial function $f_{0} \in L^{2}(Z)$, there exists at most one weak solution $f \in L^{1}(0, T$; $\left.L^{2}(Z)\right)$ of the nonautonomous transport equation

$$
\left\{\begin{aligned}
\partial_{t} f+\operatorname{div}_{\mathbf{x}}(f \mathbf{v})+\operatorname{div}_{\mathbf{y}}(f \widetilde{\mathbf{g}}(t)) & =\widetilde{u}(t) f+\widetilde{w}(t) \quad \text { in }[0, T], \\
f(0) & =f_{0}
\end{aligned}\right.
$$

In our problem we make assumptions about $\partial_{\mathbf{y}} \mathbf{g}$, but want to avoid any regularity hypotheses of $\partial_{\mathbf{x}} \mathbf{g}, \partial_{\mathbf{v}} \mathbf{g}$. Transport equation (26) can still be handled because only the coefficient of the divergence term w.r.t. $\mathbf{x}$ is simple (namely $\mathbf{v}$ ) and the divergence term w.r.t. $\mathbf{v}$ even vanishes. In short, due to this special structure, every weak solution in Proposition 5.7 proves to be a "renormalised" solution (in the sense of DiPerna and Lions [19]) and so, it is unique.

The proof is essentially based on the smoothing arguments of Le Bris and Lions in their article [40] about "transport equations with partially $W^{1,1}$ velocities". The norms in $L^{1}, L^{\infty}$ used there have just to be replaced by the $L^{2}$ norm as indicated in [19].

Remark 5.8 Weak (possibly measure-valued) solutions to linear transport equations and the relation to their (generalised) flow along ODEs (with discontinuous coefficients) belong to the current fields of research in analysis (see, e.g., [1, 2, 3, 11, 45, 47] and references therein). Existence of solutions can usually be proved by means of smoothing coefficients.

Uniqueness of weak solutions, however, proves to be a very challenging topic. [45, Theorem 5.10] exemplifies how rather weak assumptions about the divergence of the vector fields ensure the uniqueness of weak solutions to homogeneous transport equations if their values are nonnegative bounded Radon measures on the Euclidean space. In [3], however, it is pointed out as an open question whether similar uniqueness results also hold for signed measures (as values of weak solutions) - unless the vector field is assumed to fulfil an Osgood-type condition.

The following lemmas clarify the steps of the proof. The first one specifies the differential equation which the weak solution still satisfies after mollifying w.r.t. $\mathbf{x}, \mathbf{y}$. It provides the key tool for proving the second lemma concerning the absolute value of weak solutions.

Lemma 5.9 Under the assumptions of Proposition 5.7, let $f:[0, T] \longrightarrow L^{2}(Z)$ be any weak solution of initial value problem (26). Furthermore fix any smooth $\varphi:[0, \infty[\longrightarrow[0,1]$ with compact support in $\left[0,1\left[\right.\right.$ and $\varphi(\cdot)=1$ close to 0 , consider the Dirac sequences $\left(\check{\rho}_{l}\right)_{l \in \mathbb{N}},\left(\rho_{k}\right)_{k \in \mathbb{N}}$ (of mollifiers) with respect to $\mathbf{x}, \mathbf{y}$

$$
\begin{aligned}
& \rho_{k}: Y=\mathbb{R}^{d} \longrightarrow\left[0, \infty\left[, \quad \mathbf{y} \longmapsto \operatorname{const}\left(d,\|\varphi\|_{L^{1}([0, \infty[)}\right) k^{d} \cdot \varphi(k|\mathbf{y}|),\right.\right. \\
& \check{\rho}_{l}: \quad \mathbb{R}^{n} \longrightarrow\left[0, \infty\left[, \quad \mathbf{x} \longmapsto \operatorname{const}\left(n,\|\varphi\|_{L^{1}([0, \infty[)}\right) l^{n} \cdot \varphi(l|\mathbf{x}|)\right. \text {, }\right.
\end{aligned}
$$


and set for $k, l \in \mathbb{N}$ (by means of successive convolution w.r.t. $\mathbf{y}$ and $\mathbf{x}$ )

$$
\begin{aligned}
& f_{k, l}:[0, T] \times Z \longrightarrow \mathbb{R}, \\
& (t, \mathbf{x}, \mathbf{v}, \mathbf{y}) \longmapsto\left(f(t) \stackrel{\mathbf{y}}{*} \rho_{k}\right) \stackrel{\text { x }}{*} \check{\rho}_{l} \stackrel{\text { Def. }}{=} \int_{\mathbb{R}^{n}}\left(\int_{Y} f(t, \widetilde{\mathbf{x}}, \mathbf{v}, \widetilde{\mathbf{y}}) \rho_{k}(\mathbf{y}-\widetilde{\mathbf{y}}) d \widetilde{\mathbf{y}}\right) \cdot \check{\rho}_{l}(\mathbf{x}-\widetilde{\mathbf{x}}) d \widetilde{\mathbf{x}} .
\end{aligned}
$$

Then each function $f_{k, l}$ is a weak solution of the transport equation

$$
\partial_{t} f_{k, l}+\operatorname{div}_{\mathbf{x}}\left(f_{k, l} \mathbf{v}\right)+\operatorname{div}_{\mathbf{y}}\left(f_{k, l} \widetilde{\mathbf{g}}(t)\right)=\widetilde{u}(t) f_{k, l}+\left(\widetilde{w}(t) \stackrel{\mathbf{y}}{*} \rho_{k}\right) \stackrel{\mathbf{x}}{*} \check{\rho}_{l}+\epsilon_{k, l}(t)
$$

in $[0, T]$ with a residual function $\epsilon_{k, l}:[0, T] \longrightarrow L^{2}(Z)$ satisfying

$$
\lim _{k \rightarrow \infty} \lim _{l \rightarrow \infty}\left\|\epsilon_{k, l}\right\|_{L^{1}\left(0, T ; L^{2}(Z)\right)}=0 .
$$

Lemma 5.10 Under the assumptions of Proposition 5.7, let $f:[0, T] \longrightarrow L^{2}(Z)$ be a weak solution of initial value problem (26) with $\widetilde{w} \equiv 0$. For $\beta \in W^{1, \infty}(\mathbb{R})$ suppose $\beta(0)=0$.

Then the composition $f_{\beta}:=\beta \circ f:[0, T] \longrightarrow L^{2}(Z)$ satisfies the following nonautonomous transport equation in the distributional sense

$$
\partial_{t} f_{\beta}+\operatorname{div}_{\mathbf{x}}\left(f_{\beta} \mathbf{v}\right)+\operatorname{div}_{\mathbf{y}}\left(f_{\beta} \widetilde{\mathbf{g}}(t)\right)=\operatorname{div}_{\mathbf{y}} \widetilde{\mathbf{g}}(t) \cdot f_{\beta}+\beta^{\prime}(f) f \cdot\left(\widetilde{u}(t)-\operatorname{div}_{\mathbf{y}} \widetilde{\mathbf{g}}(t)\right) .
$$

Proof of Proposition 5.7. Let $\tilde{f} \in L^{1}\left(0, T ; L^{2}(Z)\right)$ denote the difference of any two weak solutions to initial value problem (26). Obviously, $\tilde{f}$ is a weak solution of

$$
\left\{\begin{aligned}
\partial_{t} \tilde{f}+\operatorname{div}_{\mathbf{x}}(\widetilde{f} \mathbf{v})+\operatorname{div}_{\mathbf{y}}(\widetilde{f} \widetilde{\mathbf{g}}(t)) & =\widetilde{u}(t) \widetilde{f} \text { in }[0, T], \\
\widetilde{f}(0) & =0 .
\end{aligned}\right.
$$

Fix $M>0$ arbitrarily. The auxiliary function $\mathbb{R} \longrightarrow \mathbb{R}, s \longmapsto \min \left\{s^{2}, M\right\}$ is bounded and Lipschitz continuous and so, it belongs to $W^{1, \infty}(\mathbb{R})$. As a consequence of Lemma 5.10 and [65, Corollary 2.1.8] (about weak derivatives of the minima of two Sobolev functions), the composed function $\widetilde{f}_{M}:=\min \left\{|\widetilde{f}|^{2}, M\right\} \in L^{1}\left(0, T ; L^{2}(Z)\right)$ is a weak solution of

$$
\begin{aligned}
\partial_{t} \widetilde{f}_{M}+\operatorname{div}_{\mathbf{x}}\left(\widetilde{f}_{M} \mathbf{v}\right)+\operatorname{div}_{\mathbf{y}}\left(\widetilde{f}_{M} \widetilde{\mathbf{g}}(t)\right) & = \begin{cases}|\widetilde{f}|^{2} \widetilde{u}(t) & \text { if }|\widetilde{f}|<M, \\
M \cdot \operatorname{div}_{\mathbf{y}} \widetilde{\mathbf{g}}(t) & \text { if }|\widetilde{f}| \geq M,\end{cases} \\
& = \begin{cases}\widetilde{f}_{M} \cdot \widetilde{u}(t) & \text { if }|\widetilde{f}|<M, \\
\widetilde{f}_{M} \cdot \operatorname{div}_{\mathbf{y}} \widetilde{\mathbf{g}}(t) & \text { if }|\widetilde{f}| \geq M .\end{cases}
\end{aligned}
$$

Our aim is now to verify

$$
\int_{Z} \min \left\{|\tilde{f}(t)|^{2}, M\right\} d \mathbf{z}=0
$$

for every $t \in[0, T]$ and $M>0$. 
Choose any test functions $\varphi_{1} \in C_{c}^{\infty}\left(\mathbb{R}^{n}\right), \varphi_{2} \in C_{c}^{\infty}(V), \varphi_{3} \in C_{c}^{\infty}(Y)$ with $\varphi_{1}=1, \varphi_{3}=1$ close to 0 and $0 \leq \varphi_{1}, \varphi_{2}, \varphi_{3} \leq 1$. For each $R>0$ set

$$
\psi_{R}: Z \stackrel{\text { Def. }}{=} \mathbb{R}^{n} \times V \times Y \longrightarrow \mathbb{R}, \quad(\mathbf{x}, \mathbf{v}, \mathbf{y}) \longmapsto \varphi_{1}\left(\frac{\mathbf{x}}{R}\right) \cdot \varphi_{2}(\mathbf{v}) \cdot \varphi_{3}\left(\frac{\mathbf{y}}{R}\right)
$$

Then we obtain at Lebesgue-almost every time instant $t \in[0, T]$

$$
\begin{aligned}
\partial_{t} \int_{Z} \widetilde{f}_{M} \psi_{R} d \mathbf{z}= & \int_{Z}\left(\widetilde{f}_{M} \mathbf{v} \cdot \nabla_{\mathbf{x}} \psi_{R}+\widetilde{f}_{M} \widetilde{\mathbf{g}}(t) \cdot \nabla_{\mathbf{y}} \psi_{R}\right) d \mathbf{z}+ \\
& \int_{Z} \widetilde{f}_{M}\left(\widetilde{u} \cdot \chi_{|\widetilde{f}|<M}+\operatorname{div}_{\mathbf{y}} \widetilde{\mathbf{g}}(t) \cdot \chi_{|\widetilde{f}| \geq M}\right) \psi_{R} d \mathbf{z} \\
\leq & \frac{\operatorname{const}\left(V,\|\widetilde{\mathbf{g}}\|_{L^{\infty}\left(L^{\infty}\right)}\right)}{R} \cdot \int_{Z}\left(\widetilde{f}_{M}\left|\left(\nabla_{\mathbf{x}} \varphi_{1}\right)\left(\frac{\mathbf{x}}{R}\right)\right|+\widetilde{f}_{M}\left|\left(\nabla_{\mathbf{y}} \varphi_{3}\right)\left(\frac{\mathbf{y}}{R}\right)\right|\right) d \mathbf{z}+ \\
& \left(\|\widetilde{u}\|_{L^{\infty}\left(0, T ; L^{\infty}(Z)\right)}+\left\|\partial_{\mathbf{y}} \widetilde{\mathbf{g}}\right\|_{L^{\infty}\left(0, T ; L^{\infty}\left(Z, \mathbb{R}^{d \times d}\right)\right)}\right) \cdot \int_{Z} \widetilde{f}_{M} \psi_{R} d \mathbf{z} \\
\leq & \frac{C}{R} \cdot \int_{Z}|\widetilde{f}(t)|^{2} d \mathbf{z}+C \cdot \int_{Z} \widetilde{f}_{M} \psi_{R} d \mathbf{z}
\end{aligned}
$$

with a constant $C=\operatorname{const}\left(V,\|\widetilde{u}\|_{L^{\infty}\left(L^{\infty}\right)},\|\widetilde{\mathbf{g}}\|_{L^{\infty}\left(L^{\infty}\right)},\left\|\partial_{\mathbf{y}} \widetilde{\mathbf{g}}\right\|_{L^{\infty}\left(L^{\infty}\right)}, \varphi_{1}, \varphi_{3}\right)$. Gronwall's inequality and the initial condition $\widetilde{f}_{M}(0)=|\widetilde{f}(0)|^{2}=0$ lead to the following explicit estimate for every $t \in[0, T]$

$$
\begin{aligned}
0 \leq \int_{Z} \widetilde{f}_{M}(t) \psi_{R} d \mathbf{z} & \leq \frac{C}{R} \int_{0}^{t} \int_{Z}|\widetilde{f}(t)|^{2} d \mathbf{z} d s \cdot e^{C t} \\
& \leq \frac{C}{R}\|\widetilde{f}\|_{L^{1}\left(0, T ; L^{2}(Z)\right)} \cdot e^{C T}
\end{aligned}
$$

For $R \longrightarrow \infty$, Lebesgue's theorem of dominated convergence ensures for any $t \in[0, T], M>0$

$$
0=\int_{Z} \widetilde{f}_{M}(t) d \mathbf{z}=\int_{Z} \min \left\{|\widetilde{f}(t)|^{2}, M\right\} d \mathbf{z}
$$

This subsection is closed with proofs of Lemmas 5.9 and 5.10. Although following the gist of $[19,40]$ about "renormalised solutions", we prefer a quite detailed presentation - for the sake of self-containedness.

Proof of Lemma 5.9. First we consider the convolution w.r.t. y, i.e. the function

$$
\begin{aligned}
\check{f}_{k}:[0, T] \times Z & \longrightarrow \mathbb{R}, \\
(t, \mathbf{x}, \mathbf{v}, \mathbf{y}) & \longmapsto\left(f(t) \stackrel{\text { }}{*} \rho_{k}\right)(\mathbf{x}, \mathbf{v}, \mathbf{y}) \stackrel{\text { Def. }}{=} \int_{Y} f(t, \mathbf{x}, \mathbf{v}, \widetilde{\mathbf{y}}) \rho_{k}(\mathbf{y}-\widetilde{\mathbf{y}}) d \widetilde{\mathbf{y}}
\end{aligned}
$$


for each $k \in \mathbb{N}$. Due to [13, Proposition 4.16] and the rotational symmetry of $\rho_{k}$, it satisfies for every test function $\varphi \in C_{c}^{1}(Z)$

$$
\begin{aligned}
& \partial_{t} \int_{Z} \varphi \check{f}_{k}(t) d \mathbf{z}=\partial_{t} \int_{Z} \varphi\left(f(t) \stackrel{\mathbf{y}}{*} \rho_{k}\right) d \mathbf{z} \\
& =\partial_{t} \int_{Z}\left(\varphi^{\mathbf{y}} \rho_{k}\right) f(t) d \mathbf{z} \\
& \stackrel{(26)}{=} \int_{Z}\left(f(t) \mathbf{v} \cdot \nabla_{\mathbf{x}}\left(\varphi^{*} \rho_{k}\right)+f(t) \widetilde{\mathbf{g}}(t) \cdot \nabla_{\mathbf{y}}\left(\varphi^{*} \rho_{k}\right)\right) d \mathbf{z}+ \\
& \int_{Z}(\widetilde{u}(t) f(t)+\widetilde{w}(t))\left(\varphi \stackrel{\mathbf{y}}{*} \rho_{k}\right) d \mathbf{z} \\
& =\int_{Z}\left(f(t) \mathbf{v} \cdot\left(\nabla_{\mathbf{x}}{\left.\stackrel{\mathrm{y}}{*} \rho_{k}\right)}+f(t) \widetilde{\mathbf{g}}(t) \cdot\left(\left(\nabla_{\mathbf{y}} \varphi\right) \stackrel{\mathbf{y}}{*} \rho_{k}\right)\right) d \mathbf{z}+\right. \\
& \int_{Z}(\widetilde{u}(t) f(t)+\widetilde{w}(t))\left(\varphi \stackrel{\mathbf{y}}{*} \rho_{k}\right) d \mathbf{z} \\
& =\int_{Z}\left(\left(f(t) \stackrel{\text { }}{*} \rho_{k}\right) \mathbf{v} \cdot \nabla_{\mathbf{x}} \varphi+(f(t) \widetilde{\mathbf{g}}(t)) \stackrel{\mathbf{y}}{*} \rho_{k} \cdot \nabla_{\mathbf{y}} \varphi\right) d \mathbf{z}+ \\
& \int_{Z}\left((\widetilde{u}(t) f(t)) \stackrel{\mathbf{y}}{*} \rho_{k}+\widetilde{w}(t) \stackrel{\mathbf{y}}{*} \rho_{k}\right) \varphi d \mathbf{z} .
\end{aligned}
$$

Hence $\check{f}_{k}$ is a weak solution of

$$
\left\{\begin{aligned}
\partial_{t} \check{f}_{k}+\operatorname{div}_{\mathbf{x}}\left(\check{f}_{k} \mathbf{v}\right)+\operatorname{div}_{\mathbf{y}}\left(\check{f}_{k} \widetilde{\mathbf{g}}(t)\right) & =\widetilde{u}(t) \check{f}_{k}+\widetilde{w}(t) \stackrel{\text { * }}{*} \rho_{k}+\check{\epsilon}_{k}(t) \quad \text { in }[0, T] \\
\check{f}_{k}(0) & =f_{0} * \rho_{k} \in L^{2}(Z)
\end{aligned}\right.
$$

with $\check{\epsilon}_{k}(t):=\operatorname{div}_{\mathbf{y}}\left(\left(f(t) \stackrel{\mathbf{y}}{*} \rho_{k}\right) \widetilde{\mathbf{g}}(t)-(f(t) \widetilde{\mathbf{g}}(t)) \stackrel{\mathbf{y}}{*} \rho_{k}\right)+(\widetilde{u}(t) f(t)) \stackrel{\mathbf{y}}{*} \rho_{k}-\widetilde{u}(t)\left(f(t) \stackrel{\mathbf{y}}{*} \rho_{k}\right)$

The next step is to prove $\lim _{k \rightarrow \infty}\left\|\check{\epsilon}_{k}\right\|_{L^{1}\left(0, T ; L^{2}(Z)\right)}=0$.

Indeed, the standard rules of convolution and Dirac sequences a.k.a. mollifiers (see, e.g., [13, $\S 4.4]$, [38, Chapter VIII]) imply for each $t \in[0, T]$ and $k \longrightarrow \infty$

$$
\begin{aligned}
& \left\|(\widetilde{u}(t) f(t)) \stackrel{\mathbf{y}}{*} \rho_{k}-\widetilde{u}(t) f(t)\right\|_{L^{2}(Z)} \longrightarrow 0, \quad\left\|(\widetilde{u}(t) f(t)) \stackrel{\mathbf{y}}{*} \rho_{k}\right\|_{L^{2}(Z)} \leq\|\widetilde{u}(t) f(t)\|_{L^{2}(Z)}, \\
& \left\|\widetilde{u}(t)\left(f(t) \stackrel{\mathbf{y}}{*} \rho_{k}\right)-\widetilde{u}(t) f(t)\right\|_{L^{2}(Z)} \longrightarrow 0, \quad\left\|\widetilde{u}(t)\left(f(t) \stackrel{\mathbf{y}}{*} \rho_{k}\right)\right\|_{L^{2}(Z)} \leq\|\widetilde{u}\|_{L^{\infty}\left(L^{\infty}(Z)\right)}\|f(t)\|_{L^{2}(Z)}
\end{aligned}
$$

Hence Lebesgue's theorem of dominated convergence implies

$$
(\widetilde{u} f) \stackrel{\mathbf{y}}{*} \rho_{k}-\widetilde{u}\left(f \stackrel{\mathbf{y}}{*} \rho_{k}\right) \longrightarrow 0 \quad \text { in } L^{1}\left(0, T ; L^{2}(Z)\right) \quad \text { for } k \longrightarrow \infty .
$$


Furthermore we conclude from $\partial_{j} \rho_{k}(\mathbf{y})=C k^{n+1} \varphi^{\prime}(k|\mathbf{y}|) \frac{y_{j}}{|\mathbf{y}|}$ for all $\mathbf{y} \in \mathbb{R}^{d}$ with a constant $C$ depending just on $d,\|\varphi\|_{L^{1}([0, \infty[)}$ that

$$
\begin{aligned}
& \operatorname{div}_{\mathbf{y}}\left(\left(f(t) \stackrel{\mathbf{y}}{*} \rho_{k}\right) \widetilde{\mathbf{g}}(t)-(f(t) \widetilde{\mathbf{g}}(t)) \stackrel{\mathbf{y}}{*} \rho_{k}\right) \\
& =\left(f \stackrel{\mathbf{y}}{*} \rho_{k}\right) \operatorname{div}_{\mathbf{y}} \widetilde{\mathbf{g}}(t)+\nabla_{\mathbf{y}}\left(f \stackrel{\mathbf{y}}{*} \rho_{k}\right) \cdot \widetilde{\mathbf{g}}(t)-\sum_{j=1}^{d} \partial_{y_{j}}\left(\left(f(t) \widetilde{\mathbf{g}}_{j}(t)\right) \stackrel{\mathbf{y}}{*} \rho_{k}\right)
\end{aligned}
$$

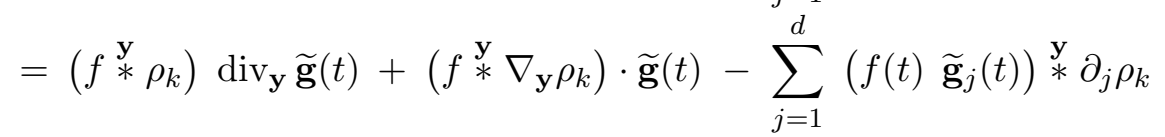

$$
\begin{aligned}
& =\left(f \stackrel{\mathbf{y}}{*} \rho_{k}\right) \operatorname{div}_{\mathbf{y}} \widetilde{\mathbf{g}}(t)+\sum_{j=1}^{d}\left(\widetilde{\mathbf{g}}_{j}(t)\left(f(t) \stackrel{\mathbf{y}}{*} \partial_{j} \rho_{k}\right)-\left(\widetilde{\mathbf{g}}_{j}(t) f(t)\right) \stackrel{\mathbf{y}}{*} \partial_{j} \rho_{k}\right) \\
& =\left(f \stackrel{\mathbf{y}}{*} \rho_{k}\right) \operatorname{div}_{\mathbf{y}} \widetilde{\mathbf{g}}(t)+\sum_{j=1}^{d} \int_{Y}\left(\widetilde{\mathbf{g}}_{j}(t, \mathbf{x}, \mathbf{v}, \mathbf{y}) \quad f(t, \mathbf{x}, \mathbf{v}, \mathbf{y}-\widetilde{\mathbf{y}})-\right. \\
& \left.\widetilde{\mathbf{g}}_{j}(t, \mathbf{x}, \mathbf{v}, \mathbf{y}-\widetilde{\mathbf{y}}) f(t, \mathbf{x}, \mathbf{v}, \mathbf{y}-\widetilde{\mathbf{y}})\right) \partial_{j} \rho_{k}(\widetilde{\mathbf{y}}) d \widetilde{\mathbf{y}} \\
& =\left(f \stackrel{\mathbf{y}}{*} \rho_{k}\right) \operatorname{div}_{\mathbf{y}} \widetilde{\mathbf{g}}(t)+\sum_{j=1}^{d} \int_{Y}\left(\widetilde{\mathbf{g}}_{j}(t, \mathbf{x}, \mathbf{v}, \mathbf{y}) \quad f(t, \mathbf{x}, \mathbf{v}, \mathbf{y}-\widetilde{\mathbf{y}})-\right. \\
& \left.\widetilde{\mathbf{g}}_{j}(t, \mathbf{x}, \mathbf{v}, \mathbf{y}-\widetilde{\mathbf{y}}) f(t, \mathbf{x}, \mathbf{v}, \mathbf{y}-\widetilde{\mathbf{y}})\right) C k^{n+1} \varphi^{\prime}(k|\widetilde{\mathbf{y}}|) \frac{\widetilde{y}_{j}}{\widetilde{\mathbf{y}} \mid} d \widetilde{\mathbf{y}} \\
& =\left(f \stackrel{\mathbf{y}}{*} \rho_{k}\right) \operatorname{div}_{\mathbf{y}} \widetilde{\mathbf{g}}(t)+\sum_{j=1}^{d} \int_{Y} \frac{\widetilde{\mathbf{g}}_{j}(t, \mathbf{x}, \mathbf{v}, \mathbf{y})-\widetilde{\mathbf{g}}_{j}(t, \mathbf{x}, \mathbf{v}, \mathbf{y}-\widetilde{\mathbf{y}})}{1 / k} \\
& \cdot f(t, \mathbf{x}, \mathbf{v}, \mathbf{y}-\widetilde{\mathbf{y}}) \quad C k^{n} \varphi^{\prime}(k|\widetilde{\mathbf{y}}|) \frac{\widetilde{y}_{j}}{|\widetilde{\mathbf{y}}|} d \widetilde{\mathbf{y}} .
\end{aligned}
$$

Extending the standard calculation in [13, Theorem 4.15] of Young to the "partial" convolution (i.e., merely w.r.t. y), the following upper estimate results from $\operatorname{supp} \varphi^{\prime}(k|\cdot|) \subseteq \mathbb{B}_{1 / k}(0)$

$$
\begin{aligned}
& \left\|\operatorname{div}_{\mathbf{y}}\left(\left(f(t) \stackrel{\mathbf{y}}{*} \rho_{k}\right) \widetilde{\mathbf{g}}(t)-(f(t) \widetilde{\mathbf{g}}(t)) \stackrel{\mathbf{y}}{*} \rho_{k}\right)\right\|_{L^{2}(Z)} \\
& \leq\|f(t)\|_{L^{2}(Z)}\left\|\operatorname{div}_{\mathbf{y}} \widetilde{\mathbf{g}}(t)\right\|_{L^{\infty}(Z)}+\sum_{j=1}^{d}\left\|\partial_{\mathbf{y}} \widetilde{\mathbf{g}}_{j}(t)\right\|_{L^{\infty}(Z)} \quad\left\||f(t)|_{*}^{\mathbf{y}}\left(C k^{n} \varphi^{\prime}(k|\cdot|)\right)\right\|_{L^{2}(Z)} \\
& \leq\|f(t)\|_{L^{2}(Z)}\left\|\operatorname{div}_{\mathbf{y}} \widetilde{\mathbf{g}}(t)\right\|_{L^{\infty}(Z)}+C d\left\|\partial_{\mathbf{y}} \widetilde{\mathbf{g}}\right\|_{L^{\infty}\left(0, T ; L^{\infty}(Z)\right)}\|f(t)\|_{L^{2}(Z)}\left\|k^{n} \varphi^{\prime}(k|\cdot|)\right\|_{L^{1}(Y)} \\
& \leq \operatorname{const}\left(d,\|\varphi\|_{L^{1}([0, \infty[)},\left\|\varphi^{\prime}\right\|_{L^{1}([0, \infty[)}\right) \cdot\left\|\partial_{\mathbf{y}} \widetilde{\mathbf{g}}\right\|_{L^{\infty}\left(0, T ; L^{\infty}(Z)\right)}\|f(t)\|_{L^{2}(Z)} \cdot
\end{aligned}
$$

By means of a density argument, the claim

$$
\operatorname{div}_{\mathbf{y}}\left(\left(f(t) \stackrel{\mathbf{y}}{*} \rho_{k}\right) \widetilde{\mathbf{g}}(t)-(f(t) \widetilde{\mathbf{g}}(t)) \stackrel{\mathbf{y}}{*} \rho_{k}\right) \longrightarrow 0 \text { in } L^{1}\left(0, T ; L^{2}(Z)\right) \text { for } k \longrightarrow \infty
$$

now results from the corresponding convergence under the additional assumption that all functions involved are smooth. In this smooth case, however, we benefit from

$$
\begin{aligned}
& \operatorname{div}_{\mathbf{y}}\left(\left(f(t) \stackrel{\mathbf{y}}{*} \rho_{k}\right) \widetilde{\mathbf{g}}(t)\right)=\left(\left(\nabla_{\mathbf{y}} f(t)\right) \stackrel{\mathbf{y}}{*} \rho_{k}\right) \cdot \widetilde{\mathbf{g}}(t)+\left(f(t) \stackrel{\mathbf{y}}{*} \rho_{k}\right) \cdot \operatorname{div}_{\mathbf{y}} \widetilde{\mathbf{g}}(t), \\
& \operatorname{div}_{\mathbf{y}}\left((f(t) \widetilde{\mathbf{g}}(t)) \stackrel{\text { * }}{*} \rho_{k}\right)=\left(\operatorname{div}_{\mathbf{y}}(f(t) \widetilde{\mathbf{g}}(t))\right) \stackrel{\mathbf{y}}{*} \rho_{k}
\end{aligned}
$$

and each of them converges to $t \longmapsto \operatorname{div}_{\mathbf{y}}(f(t) \widetilde{\mathbf{g}}(t))$ in $L^{1}\left(0, T ; L^{2}(Z)\right)$ for $k \longrightarrow \infty$. Hence, we have completed the step verifying $\lim _{k \rightarrow \infty}\left\|\check{\epsilon}_{k}\right\|_{L^{1}\left(0, T ; L^{2}(Z)\right)}=0$. 
Now the additional regularisation w.r.t. $\mathbf{x}$ is considered. Following essentially the same steps as to initial value problem $(29)$ for $\check{f}_{k}$, the function $f_{k, l}: t \longmapsto\left(f(t) \stackrel{\mathbf{y}}{*} \rho_{k}\right) \stackrel{\text { * }}{*} \check{\rho}_{l}$ proves to be a weak solution of

$$
\left\{\begin{aligned}
\partial_{t} f_{k, l}+\operatorname{div}_{\mathbf{x}}\left(\check{f}_{k} \mathbf{v}\right)+\operatorname{div}_{\mathbf{y}}\left(f_{k, l} \widetilde{\mathbf{g}}(t)\right) & =\widetilde{u}(t) f_{k, l}+\left(\widetilde{w}(t) \stackrel{\mathbf{y}}{*} \rho_{k}\right) * \check{\rho}_{l}+\epsilon_{k, l}(t) \quad \text { in }[0, T] \\
f_{k, l}(0) & =\left(f_{0} \stackrel{\mathbf{y}}{*} \rho_{k}\right) \stackrel{\text { x }}{*} \check{\rho}_{l} \in L^{2}(Z)
\end{aligned}\right.
$$

with the residual function $\epsilon_{k, l}:[0, T] \longrightarrow L^{2}(Z)$,

$$
\begin{aligned}
\epsilon_{k, l}(t):=\check{\epsilon}_{k} * \check{\rho}_{l} & +\operatorname{div}_{\mathbf{y}}\left(\left(\check{f}_{k}(t) \stackrel{\mathbf{x}}{*} \check{\rho}_{l}\right) \widetilde{\mathbf{g}}(t)-\left(\check{f}_{k}(t) \underset{\mathbf{g}}{\mathbf{\mathbf { g }}}(t)\right) * \check{\rho}_{l}\right) \\
& +\left(\widetilde{u}(t) \check{f}_{k}(t)\right) \stackrel{\mathbf{x}}{*} \check{\rho}_{l}-\widetilde{u}(t)\left(\check{f}_{k}(t) \stackrel{\mathbf{x}}{*} \check{\rho}_{l}\right) .
\end{aligned}
$$

It remains to prove $\lim _{k \rightarrow \infty} \lim _{l \rightarrow \infty}\left\|\epsilon_{k, l}\right\|_{L^{1}\left(0, T ; L^{2}(Z)\right)}=0$. We consider the three terms of $\epsilon_{k, l}(t)$ successively. Due to the general convergence properties of mollifiers,

$$
\check{\epsilon}_{k} \stackrel{\mathbf{x}}{*} \check{\rho}_{l} \longrightarrow \check{\epsilon}_{k} \quad \text { in } L^{1}\left(0, T ; L^{2}(Z)\right) \text { for } l \longrightarrow \infty \text { and } k \in \mathbb{N} \text { fixed. }
$$

Furthermore, mollifying w.r.t. $\mathbf{y}$ implies for $\check{f}_{k}(t) \stackrel{\text { Def. }}{=} f(t) \stackrel{\mathbf{y}}{*} \rho_{k}$ that the weak partial derivative $\nabla_{\mathbf{y}} \check{f}_{k}$ exists for each index $k \in \mathbb{N}$ and satisfies $\left\|\nabla_{\mathbf{y}} \check{f}_{k}(t)\right\|_{L^{2}(Z)} \leq \operatorname{const}\left(\nabla \rho_{k}\right) \cdot\|f(t)\|_{L^{2}(Z)}$, i.e. $\nabla_{\mathbf{y}} \check{f}_{k} \in L^{1}\left(0, T ; L^{2}\left(Z, \mathbb{R}^{d}\right)\right)$. Thus,

$$
\begin{aligned}
& \operatorname{div}_{\mathbf{y}}\left(\left(\check{f}_{k}(t) * \check{\rho}_{l}\right) \widetilde{\mathbf{g}}(t)-\left(\check{f}_{k}(t) \widetilde{\mathbf{g}}(t)\right) * \begin{array}{l}
\mathbf{x} \\
*
\end{array} \check{\rho}_{l}\right) \\
& =\left(\nabla_{\mathbf{y}} \check{f}_{k}(t) * \check{\rho}_{l}\right) \cdot \widetilde{\mathbf{g}}(t)+\left(\check{f}_{k}(t) * \check{\rho}_{l}\right) \cdot \operatorname{div}_{\mathbf{y}} \widetilde{\mathbf{g}}(t)-\operatorname{div}_{\mathbf{y}}\left(\check{f}_{k}(t) \widetilde{\mathbf{g}}(t)\right) * \check{\rho}_{l}
\end{aligned}
$$

is converging to 0 in $L^{2}(Z)$ for $l \longrightarrow \infty$ and every $k \in \mathbb{N}, t \in[0, T]$ fixed. For concluding its convergence in $L^{1}\left(0, T ; L^{2}(Z)\right)$ from Lebesgue's theorem of dominated convergence, we just need the following estimate for index $k \in \mathbb{N}$ fixed:

$$
\begin{aligned}
& \left\|\operatorname{div}_{\mathbf{y}}\left(\left(\check{f}_{k}(t) \stackrel{\mathbf{x}}{*} \check{\rho}_{l}\right) \widetilde{\mathbf{g}}(t)-\left(\check{f}_{k}(t) \widetilde{\mathbf{g}}(t)\right) \stackrel{\mathbf{x}}{*} \check{\rho}_{l}\right)\right\|_{L^{2}(Z)} \\
& \leq\left\|\left(\nabla_{\mathbf{y}} \check{f}_{k}(t) \stackrel{\mathbf{x}}{*} \check{\rho}_{l}\right) \cdot \widetilde{\mathbf{g}}(t)\right\|_{L^{2}(Z)}+\left\|\left(\check{f}_{k}(t) \stackrel{\mathbf{x}}{*} \check{\rho}_{l}\right) \cdot \operatorname{div}_{\mathbf{y}} \widetilde{\mathbf{g}}(t)\right\|_{L^{2}(Z)}+\left\|\operatorname{div}_{\mathbf{y}}\left(\check{f}_{k}(t) \widetilde{\mathbf{g}}(t)\right) * \check{\rho}_{l}\right\|_{L^{2}} \\
& \leq\left\|\nabla_{\mathbf{y}} \check{f}_{k}(t)\right\|_{L^{2}} \cdot\|\widetilde{\mathbf{g}}(t)\|_{L^{\infty}}+\left\|\check{f}_{k}(t)\right\|_{L^{2}} \cdot\left\|\operatorname{div}_{\mathbf{y}} \widetilde{\mathbf{g}}(t)\right\|_{L^{\infty}}+\left\|\operatorname{div}_{\mathbf{y}}\left(\check{f}_{k}(t) \widetilde{\mathbf{g}}(t)\right)\right\|_{L^{2}} \\
& \leq 2 \cdot\left(\left\|\check{f}_{k}(t)\right\|_{L^{2}(Z)}+\left\|\nabla_{\mathbf{y}} \check{f}_{k}(t)\right\|_{L^{2}\left(Z, \mathbb{R}^{d}\right)}\right) \cdot\left(\|\widetilde{\mathbf{g}}\|_{L^{\infty}\left(0, T ; L^{\infty}\left(Z, \mathbb{R}^{d}\right)\right)}+\left\|\operatorname{div}_{\mathbf{y}} \widetilde{\mathbf{g}}\right\|_{L^{\infty}\left(0, T ; L^{\infty}(Z)\right)}\right) .
\end{aligned}
$$

Finally, $\left(\widetilde{u}(t) \check{f}_{k}(t)\right) * \underset{\mathbf{x}}{*} \check{\rho}_{l}-\widetilde{u}(t)\left(\check{f}_{k}(t) \stackrel{\mathbf{x}}{*} \check{\rho}_{l}\right) \longrightarrow 0$ in $L^{1}\left(0, T ; L^{2}(Z)\right)$ for $l \longrightarrow \infty$ and $k \in \mathbb{N}$ fixed results from the theorem of dominated convergence as before.

Proof of Lemma 5.10. Let $f \in L^{1}\left(0, T ; L^{2}(Z)\right)$ be any weak solution of

$$
\left\{\begin{aligned}
\partial_{t} f+\operatorname{div}_{\mathbf{x}}(f \mathbf{v})+\operatorname{div}_{\mathbf{y}}(f \widetilde{\mathbf{g}}(t)) & =\widetilde{u}(t) f \quad \text { in }[0, T], \\
f(0) & =f_{0} .
\end{aligned}\right.
$$

Consider $f_{k, l}:[0, T] \longrightarrow L^{2}(Z), k, l \in \mathbb{N}$, defined in Lemma 5.9 by means of mollifying w.r.t. $\mathbf{y}, \mathbf{x}$ successively. Then each $f_{k, l}(t) \in L^{2}(Z)$ is partially differentiable w.r.t. $\mathbf{x}, \mathbf{y}$ and, we have $\nabla_{\mathbf{x}} f_{k, l} \in L^{1}\left(0, T ; L^{2}\left(Z, \mathbb{R}^{n}\right)\right)$ as well as $\nabla_{\mathbf{y}} f_{k, l} \in L^{1}\left(0, T ; L^{2}\left(Z, \mathbb{R}^{d}\right)\right)$. 
Originally, $\beta: \mathbb{R} \longrightarrow \mathbb{R}$ is supposed to be in $W^{1, \infty}(\mathbb{R})$ with $\beta(0)=0$. In addition, we now assume $\beta \in C^{1}(\mathbb{R})$. The composition $\beta \circ f_{k, l}$ has all its values in $L^{2}(Z)$ and, it is partially differentiable w.r.t. $\mathbf{x}, \mathbf{y}$. Furthermore fix a test function $\varphi \in C_{c}^{\infty}(Z)$ arbitrarily.

As a consequence of modified initial value problem (27) (with $\widetilde{w} \equiv 0$ ), the function

$$
[0, T] \longrightarrow \mathbb{R}, \quad t \longmapsto \int_{Z} f_{k, l}(t) \cdot \varphi d \mathbf{z}
$$

is absolutely continuous for each $k, l \in \mathbb{N}$ fixed. The Lipschitz continuity of $\beta \in C^{1}(\mathbb{R})$ now implies the absolute continuity of

$$
[0, T] \longrightarrow \mathbb{R}, \quad t \longmapsto \int_{Z} \beta\left(f_{k, l}(t)\right) \cdot \varphi d \mathbf{z}
$$

and, its weak derivative results from the generalised chain rule:

$$
\begin{aligned}
& \partial_{t} \int_{Z} \beta\left(f_{k, l}(t)\right) \cdot \varphi d \mathbf{z} \\
& \stackrel{(27)}{=} \int_{Z} \beta^{\prime}\left(f_{k, l}\right)\left(-\operatorname{div}_{\mathbf{x}}\left(f_{k, l} \mathbf{v}\right)-\operatorname{div}_{\mathbf{y}}\left(f_{k, l} \widetilde{\mathbf{g}}\right)+\widetilde{u} f_{k, l}+\epsilon_{k, l}\right) \cdot \varphi d \mathbf{z} \\
& =\int_{Z}\left(-\operatorname{div}_{\mathbf{x}}\left(\beta\left(f_{k, l}\right) \mathbf{v}\right)-\nabla_{\mathbf{y}}\left(\beta \circ f_{k, l}\right) \cdot \widetilde{\mathbf{g}}-\beta^{\prime}\left(f_{k, l}\right) f_{k, l} \cdot \operatorname{div}_{\mathbf{y}} \widetilde{\mathbf{g}}\right. \\
& \left.+\beta^{\prime}\left(f_{k, l}\right)\left(\widetilde{u} f_{k, l}+\epsilon_{k, l}\right)\right) \cdot \varphi d \mathbf{z} \\
& =\int_{Z}\left(-\operatorname{div}_{\mathbf{x}}\left(\beta\left(f_{k, l}\right) \mathbf{v}\right)-\operatorname{div}_{\mathbf{y}}\left(\beta\left(f_{k, l}\right) \widetilde{\mathbf{g}}\right)+\left(\beta\left(f_{k, l}\right)-\beta^{\prime}\left(f_{k, l}\right) f_{k, l}\right) \cdot \operatorname{div}_{\mathbf{y}} \widetilde{\mathbf{g}}\right. \\
& \left.+\beta^{\prime}\left(f_{k, l}\right)\left(\widetilde{u} f_{k, l}+\epsilon_{k, l}\right)\right) \cdot \varphi d \mathbf{z}
\end{aligned}
$$

In other words, the composition $\widetilde{f}_{k, l}:=\beta\left(f_{k, l}\right):[0, T] \longrightarrow L^{2}(Z)$ is a weak solution of the nonautonomous transport equation

$$
\begin{aligned}
\partial_{t} \widetilde{f}_{k, l}+\operatorname{div}_{\mathbf{x}}\left(\widetilde{f}_{k, l} \mathbf{v}\right)+\operatorname{div}_{\mathbf{y}}\left(\widetilde{f}_{k, l} \widetilde{\mathbf{g}}\right)= & \operatorname{div}_{\mathbf{y}} \widetilde{\mathbf{g}} \cdot \widetilde{f}_{k, l}+ \\
& \beta^{\prime}\left(f_{k, l}\right)\left(f_{k, l} \cdot\left(\widetilde{u}-\operatorname{div}_{\mathbf{y}} \widetilde{\mathbf{g}}\right)+\epsilon_{k, l}\right) .
\end{aligned}
$$

Now consider first $l \longrightarrow \infty$ (with $k \in \mathbb{N}$ fixed) and then $k \longrightarrow \infty$. Due to the double limit of $\epsilon_{k, l}$ mentioned in Lemma 5.9, $f_{\beta}:=\beta \circ f:[0, T] \longrightarrow L^{2}(Z)$ solves the claimed equation (28) in the distributional sense.

Finally, we have to dispense with the additional assumption $\beta \in C^{1}(\mathbb{R})$. For $\beta \in W^{1, \infty}(\mathbb{R})$ with $\beta(0)=0$ given, the preceding result is applied to a bounded approximating sequence $\left(\beta_{m}\right)_{m \in \mathbb{N}}$ in $W^{1, \infty}(\mathbb{R}) \cap C^{1}(\mathbb{R})$ such that $\beta_{m}(0)=0$ for each $m \in \mathbb{N}$ and $\beta_{m} \longrightarrow \beta$ and $\beta_{m}^{\prime} \longrightarrow \beta^{\prime}$ Lebesgue-almost everywhere in $\mathbb{R}$ for $m \longrightarrow \infty$. Then the claimed equation (28) for $f_{\beta}:=\beta \circ f:[0, T] \longrightarrow L^{2}(Z)$ even holds for any $\beta \in W^{1, \infty}(\mathbb{R})$ with $\beta(0)=0$ (in the distributional sense). 
5.3 Linear problem with "more regular" coefficients $\mathrm{g} \in C_{c}^{1}\left(Z, \mathbb{R}^{d}\right), u \in C^{1}(Z)$ $\cap W^{1, \infty}(Z)$ : Existence of solutions

\section{Proposition 5.11}

Suppose $\mathbf{g} \in C_{c}^{1}\left(Z, \mathbb{R}^{d}\right), u \in C^{1}(Z) \cap W^{1, \infty}(Z)$ and $w \in L^{2}(Z)$.

Then for any initial function $f_{0} \in L^{2}(Z)$ and period $T>0$, there exists a weak solution $f:[0, T] \longrightarrow L^{2}(Z)$ of

$$
\left\{\begin{aligned}
\partial_{t} f+\operatorname{div}_{\mathbf{x}}(f \mathbf{v})+\operatorname{div}_{\mathbf{y}}(f \mathbf{g}) & =u f+w \quad \text { in }[0, T], \\
f(0) & =f_{0}
\end{aligned}\right.
$$

in the following sense: $f:[0, T] \longrightarrow L^{2}(Z)$ is weakly continuous with $f(0)=f_{0}$ and it holds for any $0 \leq t_{1}<t_{2} \leq T, \varphi \in C_{c}^{1}(Z)$

$$
\begin{aligned}
\int_{Z} \varphi\left(f\left(t_{2}\right)-f\left(t_{1}\right)\right) d \mathbf{z}= & \int_{t_{1}}^{t_{2}} \int_{Z}\left(f(s, \cdot)\left(\mathbf{v} \cdot \nabla_{\mathbf{x}} \varphi+\mathbf{g} \cdot \nabla_{\mathbf{y}} \varphi+u \varphi\right)\right) d \mathbf{z} d s \\
& +\left(t_{2}-t_{1}\right) \int_{Z} \varphi w d \mathbf{z} .
\end{aligned}
$$

This solution can be represented as

$$
\int_{Z} \varphi \cdot f(t) d \mathbf{z}=\int_{Z}\left(\psi_{t, \varphi}(0 ; \cdot) f_{0}+w \int_{0}^{t} \psi_{t, \varphi}(s ; \cdot) d s\right) d \mathbf{z}
$$

with $\psi_{t, \varphi} \in C^{1}([0, t] \times Z)$ denoting the unique solution to the adjoint problem (39) below. Moreover it satisfies the a priori estimate with the notation $[r]^{-}:=\min \{r, 0\}$ for $r \in \mathbb{R}$

$$
\|f(t)\|_{L^{2}(Z)} \leq\left(\left\|f_{0}\right\|_{L^{2}(Z)}+\|w\|_{L^{2}(Z)} t\right) \cdot e^{\left(\left\|\left[\operatorname{div}_{\mathbf{y}} \mathbf{g}\right]^{-}\right\|_{\left.L^{\infty}+\|u\|_{L^{\infty}}\right) \cdot t}\right.} .
$$

Remark 5.12 The characterisation of "weak solution" in Proposition 5.11 differs slightly from the standard definition, but it implies successively by means of approximation:

(1.) For any $\varphi \in C_{c}^{1}(Z)$, the function $[0, T] \longrightarrow \mathbb{R}, t \longmapsto \int_{Z} \varphi f(t) d \mathbf{z}$ is Lipschitz continuous. Indeed the image $f([0, T]) \subseteq L^{2}(Z)$ is weakly compact, hence bounded and so the claimed Lipschitz continuity results from the detailed reformulation (32) of weak solutions.

(2.) For any test function $\phi \in C_{c}^{\infty}([0, T] \times Z)$ depending on time additionally, it holds

$$
\begin{aligned}
\int_{Z}\left(\phi(T, \mathbf{z}) f(T, \mathbf{z})-\phi(0, \mathbf{z}) f_{0}(\mathbf{z})\right) d \mathbf{z} \\
=\int_{0}^{T} \int_{Z}\left(f(s, \mathbf{z})\left(\mathbf{v} \cdot \nabla_{\mathbf{x}} \phi(s, \mathbf{z})+\mathbf{g} \cdot \nabla_{\mathbf{y}} \phi(s, \mathbf{z})+u \phi(s, \mathbf{z})\right)\right) d \mathbf{z} d s \\
\quad+\int_{0}^{T} \int_{Z}\left(\phi(s, \mathbf{z}) w+\partial_{s} \phi(s, \mathbf{z}) f(s, \mathbf{z})\right) d \mathbf{z} d s .
\end{aligned}
$$


Remark 5.13 In regard to initial value problem (31), the existence of weak solutions in $L^{\infty}\left(0, T ; L^{2}(Z)\right)$ has already been verified under weaker assumptions about $\mathbf{g}, \operatorname{div}_{\mathbf{y}} \mathbf{g}, \quad u$ (see, e.g.,[19, Proposition II.1] with the modified regularisation presented in [40]).

For investigating the dependence of solutions on initial data and coefficients, however, we prefer supplementary uniqueness and need an appropriate representation such as in (33).

\section{Lemma 5.14 (The flow along the nonautonomous ODE with vector field $\mathrm{g}$ )} For every $\alpha \in \mathbb{R}$ and $\mathbf{g} \in L_{\mathrm{loc}}^{1}\left(Z, \mathbb{R}^{d}\right)$ with $\partial_{\mathbf{y}} \mathbf{g} \in L^{\infty}\left(Z, \mathbb{R}^{d \times d}\right)$, there exists a function

$$
\mathfrak{Y}_{\alpha, \mathbf{g}}: \quad\left[0, \infty\left[\times Z \longrightarrow Y, \quad(t ; \mathbf{x}, \mathbf{v}, \mathbf{y}) \longmapsto \mathfrak{Y}_{\alpha, \mathbf{g}}(t ; \mathbf{x}, \mathbf{v}, \mathbf{y})\right.\right.
$$

induced by the unique Carathéodory solutions to the nonautonomous differential equation

$$
\left\{\begin{aligned}
\partial_{t} \mathfrak{Y}_{\alpha, \mathbf{g}}(t ; \mathbf{x}, \mathbf{v}, \mathbf{y}) & =\alpha \cdot \mathbf{g}\left(\mathbf{x}+\alpha \mathbf{v} t, \mathbf{v}, \mathfrak{Y}_{\alpha, \mathbf{g}}(t ; \mathbf{x}, \mathbf{v}, \mathbf{y})\right) \quad \text { in }[0, \infty[, \\
\mathfrak{Y}_{\alpha, \mathbf{g}}(0 ; \mathbf{x}, \mathbf{v}, \mathbf{y}) & =\mathbf{y} .
\end{aligned}\right.
$$

Moreover, $\mathfrak{Y}_{\alpha, \mathbf{g}}(t ; \mathbf{x}, \mathbf{v}, \cdot): Y \longrightarrow Y$ is a Lipschitz continuous homeomorphism for each $t \geq 0$, $\mathbf{x} \in \mathbb{R}^{n}, \mathbf{v} \in V$ and it satisfies with the notation $[r]^{+}:=\max \{r, 0\},[r]^{-}:=\min \{r, 0\}$ for $r \in \mathbb{R}$

$$
\begin{aligned}
\left\|\partial_{\mathbf{y}} \mathfrak{Y}_{\alpha, \mathbf{g}}(t ; \cdot)\right\|_{L^{\infty}(Z)} & \leq e^{\operatorname{const}(n,|\alpha|)\left(1+\left\|\partial_{\mathbf{y}} \mathbf{g}\right\|_{L^{\infty}}\right) \cdot t} \\
\frac{\partial}{\partial t} \operatorname{det} \partial_{\mathbf{y}} \mathfrak{Y}_{\alpha, \mathbf{g}}(t ; \cdot) & =\left.\alpha \cdot\left(\operatorname{div}_{\mathbf{y}} \mathbf{g}\right)\right|_{\left(\mathbf{x}+\alpha \mathbf{v} t, \mathbf{v}, \mathfrak{Y}_{\alpha, \mathbf{g}}(t ; \mathbf{x}, \mathbf{v}, \mathbf{y})\right)} \cdot \operatorname{det} \partial_{\mathbf{y}} \mathfrak{Y}_{\alpha, \mathbf{g}}(t ; \cdot) \\
e^{-|\alpha|\left\|\left[\operatorname{div}_{\mathbf{y}} \mathbf{g}\right]^{-}\right\|_{L^{\infty}} \cdot t} & \leq \operatorname{det} \partial_{\mathbf{y}} \mathfrak{Y}_{\alpha, \mathbf{g}}(t ; \cdot) \leq e^{|\alpha|\left\|\left[\operatorname{div}_{\mathbf{y}} \mathbf{g}\right]^{+}\right\|_{L^{\infty}} \cdot t} .
\end{aligned}
$$

\section{Lemma 5.15 (The flow along the "full" autonomous ODE)}

Suppose $\alpha \in \mathbb{R}, \mathbf{g} \in L_{\mathrm{loc}}^{1}\left(Z, \mathbb{R}^{d}\right)$ and $\partial_{\mathbf{y}} \mathbf{g} \in L^{\infty}\left(Z, \mathbb{R}^{d \times d}\right)$. Then,

$$
\mathfrak{Z}_{\alpha, \mathbf{g}}: \quad\left[0, \infty\left[\times Z \longrightarrow Z, \quad(t ; \mathbf{x}, \mathbf{v}, \mathbf{y}) \longmapsto\left(\mathbf{x}+\alpha \mathbf{v} t, \mathbf{v}, \mathfrak{Y}_{\alpha, \mathbf{g}}(t ; \mathbf{x}, \mathbf{v}, \mathbf{y})\right)\right.\right.
$$

describes the unique Carathéodory solutions to the autonomous ordinary differential equation

$$
\left\{\begin{aligned}
\partial_{t} \mathfrak{Z}_{\alpha, \mathbf{g}}(t ; \mathbf{x}, \mathbf{v}, \mathbf{y}) & =\alpha\left(\mathbf{v}, 0, \mathbf{g}\left(\mathfrak{Z}_{\alpha, \mathbf{g}}(t ; \mathbf{x}, \mathbf{v}, \mathbf{y})\right) \quad \text { in }[0, \infty[\right. \\
\mathfrak{Z}_{\alpha, \mathbf{g}}(0 ; \mathbf{x}, \mathbf{v}, \mathbf{y}) & =(\mathbf{x}, \mathbf{v}, \mathbf{y})
\end{aligned}\right.
$$

and so, it is a semigroup on $Z \stackrel{\text { Def. }}{=} \mathbb{R}^{n} \times V \times Y$.

If we assume $\mathbf{g} \in C_{c}^{1}\left(Z, \mathbb{R}^{d}\right)$ in addition, then $\mathfrak{Z}_{\alpha, \mathbf{g}}(t, \cdot): Z \longrightarrow Z$ is continuously differentiable for each $t \in[0, \infty[$ and satisfies

$$
\left\|\partial_{\mathbf{z}} \mathfrak{Z}_{\alpha, \mathbf{g}}(t ; \cdot)\right\|_{L^{\infty}(Z)} \leq e^{\operatorname{const}(n,|\alpha|)\left(1+\|D \mathbf{g}\|_{L^{\infty}}\right) \cdot t} .
$$

In particular, each function $\mathfrak{Z}_{\alpha, \mathbf{g}}(t, \cdot): Z \longrightarrow Z, t \in[0, \infty[$, is a diffeomorphism with

$$
\begin{aligned}
\frac{\partial}{\partial t} \operatorname{det} \partial_{\mathbf{z}} \mathfrak{Z}_{\alpha, \mathbf{g}}(t ; \cdot) & =\alpha \cdot\left(\operatorname{div}_{\mathbf{y}} \mathbf{g}\right)\left(\mathfrak{Z}_{\alpha, \mathbf{g}}(t ; \cdot)\right) \cdot \operatorname{det} \partial_{\mathbf{z}} \mathfrak{Z}_{\alpha, \mathbf{g}}(t ; \cdot) \\
e^{-|\alpha|\left\|\left[\operatorname{div}_{\mathbf{y}} \mathbf{g}\right]^{-}\right\|_{L^{\infty}} \cdot t} \leq \operatorname{det} \partial_{\mathbf{z}} \mathfrak{Z}_{\alpha, \mathbf{g}}(t ; \cdot) & \leq e^{|\alpha|\left\|\left[\operatorname{div}_{\mathbf{y}} \mathbf{g}\right]^{-}\right\|_{L^{\infty}} \cdot t} .
\end{aligned}
$$

Remark 5.16 These properties of $\mathfrak{Y}_{\alpha, \mathbf{g}}, \mathfrak{Z}_{\alpha, \mathrm{g}}$ result from the standard theory about ordinary differential equations (see, e.g., [30, § V.3], [2, Remark 6.3]). 


\section{Lemma 5.17}

Suppose $\mathbf{g} \in C_{c}^{1}\left(Z, \mathbb{R}^{d}\right)$ and $u \in C^{1}(Z) \cap W^{1, \infty}(Z)$.

Then for any $t \in[0, T]$ and $\varphi \in C_{c}^{1}(Z)$, there is a unique solution $\psi_{t, \varphi}=\psi \in C^{1}([0, t] \times Z)$ of

$$
\left\{\begin{array}{rlll}
\partial_{t} \psi+\mathbf{v} \cdot \nabla_{\mathbf{x}} \psi+\mathbf{g} \cdot \nabla_{\mathbf{y}} \psi+u \psi & = & 0 & \text { in }[0, t] \times Z, \\
\psi(t) & = & \varphi & \text { in }
\end{array}\right.
$$

i.e.

$$
\psi(s ; \mathbf{x}, \mathbf{v}, \mathbf{y})=\varphi\left(\mathfrak{Z}_{1, \mathbf{g}}(t-s ; \cdot)\right) \cdot \exp \left(\int_{0}^{t-s} u\left(\mathfrak{Z}_{1, \mathbf{g}}(r ; \cdot)\right) d r\right) .
$$

Moreover, for every $0 \leq s \leq t \leq T$ and $\varphi \in C_{c}^{1}\left(\mathbb{R}^{n}\right)$, the following a priori estimate holds

$$
\left\|\psi_{t, \varphi}(s ; \cdot)\right\|_{L^{2}(Z)} \leq\|\varphi\|_{L^{2}(Z)} \cdot e^{\left(\left\|\left[\operatorname{div}_{\mathbf{y}} \mathbf{g}\right]^{-}\right\|_{L^{\infty}+\|u\|_{\left.L^{\infty}\right) \cdot t}}\right.}
$$

Proof of Lemma 5.17. Choose $t \in[0, T]$ and $\varphi \in C_{c}^{1}(Z)$. The method of characteristics provides the explicit solution $\phi \in C^{1}([0, t] \times Z)$ of the semilinear initial value problem

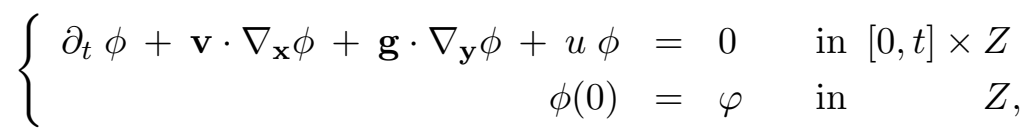

namely

$$
\phi(s ; \cdot)=\varphi\left(\mathfrak{Z}_{-1, \mathbf{g}}(s ; \cdot)\right) \cdot \exp \left(-\int_{0}^{s} u\left(\mathfrak{Z}_{-1, \mathbf{g}}(s-r ; \cdot)\right) d r\right) .
$$

Substituting $\psi(\tau ; \mathbf{z}):=\phi(t-\tau ; \mathbf{z})$ for $\tau \in[0, t]$, we obtain the claimed solution $\psi \in C^{1}([0, t] \times Z)$ of the given transport equation with end-time condition $\psi(t, \cdot)=\varphi$ :

$$
\psi(s ; \cdot)=\varphi\left(\mathfrak{Z}_{1, \mathbf{g}}(t-s ; \cdot)\right) \cdot \exp \left(\int_{s}^{t} u\left(\mathfrak{Z}_{1, \mathbf{g}}(r-s ; \cdot)\right) d r\right) .
$$

The transformation theorem for Lebesgue integrals leads to

$$
\begin{aligned}
\int_{Z}\left|\psi_{t, \varphi}(s ; \mathbf{z})\right|^{2} d \mathbf{z} & \leq e^{2\|u\|_{L^{\infty}} \cdot(t-s)} \cdot \int_{Z}\left|\varphi\left(\mathfrak{Z}_{1, \mathbf{g}}(t-s ; \mathbf{z})\right)\right|^{2} d \mathbf{z} \\
& \leq e^{2\|u\|_{L^{\infty}} \cdot(t-s)} \cdot\left\|\operatorname{det}\left(\partial_{\mathbf{z}} \mathfrak{Z}_{1, \mathbf{g}}(t-s ; \cdot)\right)^{-1}\right\|_{L^{\infty}(Z)}\|\varphi\|_{L^{2}(Z)}^{2} \\
& \leq e^{2\|u\|_{L^{\infty} \cdot t}} \cdot e^{\left\|\left[\operatorname{div}_{\mathbf{y}} \mathbf{g}\right]^{-}\right\|_{L^{\infty}} \cdot t} \quad\|\varphi\|_{L^{2}(Z)}^{2} \cdot
\end{aligned}
$$

Proof of Proposition 5.11. For any $t \in[0, T]$ and $\varphi \in C_{c}^{1}(Z)$, we consider the solution $\psi_{t, \varphi} \in C^{1}([0, t] \times Z)$ to the semilinear end-time problem (39). Due to the a priori bound (41) in Lemma 5.17, the linear operators $C_{c}^{1}(Z) \longrightarrow C^{1}(Z)$,

$$
\begin{aligned}
\varphi & \longmapsto \psi_{t, \varphi}(s ; \cdot)=\varphi\left(\mathfrak{Z}_{1, \mathbf{g}}(t-s ; \cdot)\right) \cdot \exp \left(\int_{0}^{t-s} u\left(\mathfrak{Z}_{1, \mathbf{g}}(r ; \cdot)\right) d r\right), \\
\varphi & \longmapsto \int_{0}^{t} \psi_{t, \varphi}(s ; \cdot) d s
\end{aligned}
$$


are continuous with respect to the $L^{2}(Z)$ norm and so, they have unique continuous extensions to $L^{2}(Z)$. For each $t \in[0, T]$, select the unique function $f(t) \in L^{2}(Z)$ by means of Riesz' theorem such that for any $\varphi \in C_{c}^{1}(Z)$,

$$
\int_{Z} \varphi f(t) d \mathbf{z}=\int_{Z}\left(\psi_{t, \varphi}(0 ; \cdot) f_{0}+w \int_{0}^{t} \psi_{t, \varphi}(s ; \cdot) d s\right) d \mathbf{z} .
$$

In particular, $\|f(t)\|_{L^{2}(Z)}$ is bounded by a constant depending on $\left\|\left[\operatorname{div}_{\mathbf{y}} \mathbf{g}\right]^{-}\right\|_{L^{\infty}},\|u\|_{L^{\infty}},\|w\|_{L^{2}}$, $\left\|f_{0}\right\|_{L^{2}}$ and $T$ only:

$$
\begin{aligned}
& \|f(t)\|_{L^{2}}= \\
& \sup \left\{\int_{Z} \varphi \cdot f(t) d \mathbf{z} \mid \varphi \in L^{2}(Z),\|\varphi\|_{L^{2}} \leq 1\right\} \\
& =\quad \sup \left\{\int_{Z} \varphi \cdot f(t) d \mathbf{z} \mid \varphi \in C_{c}^{1}(Z),\|\varphi\|_{L^{2}} \leq 1\right\} \\
& =\sup \left\{\left\|\psi_{t, \varphi}(s ; \cdot)\right\|_{L^{2}(Z)}\left(\left\|f_{0}\right\|_{L^{2}(Z)}+\|w\|_{L^{2}(Z)} t\right) \mid \varphi \in C_{c}^{1}(Z),\|\varphi\|_{L^{2}} \leq 1, \quad s \leq t\right\} \\
& \stackrel{(41)}{\leq} e^{\left(\left\|\left[\operatorname{div}_{\mathbf{y}} \mathbf{g}\right]^{-}\right\|_{\left.L^{\infty}+\|u\|_{L} \infty\right) \cdot t}\right.} \cdot\left(\left\|f_{0}\right\|_{L^{2}(Z)}+\|w\|_{L^{2}(Z)} t\right) .
\end{aligned}
$$

This leads to the bounded function $f:[0, T] \longrightarrow L^{2}(Z)$, which we now prove to be a weak solution of problem (31).

For any test function $\varphi \in C_{c}^{1}(Z)$ with compact support, the function $\Phi:[0, T] \longrightarrow \mathbb{R}, t \longmapsto$ $\int_{Z} \varphi f(t) d \mathbf{z}$ is absolutely continuous with

$$
\begin{aligned}
\Phi^{\prime}(t)= & \frac{d}{d t} \int_{Z}\left(f_{0}(\mathbf{z}) \varphi\left(\mathfrak{Z}_{1, \mathbf{g}}(t ; \mathbf{z})\right) e^{\int_{0}^{t} u\left(\mathfrak{Z}_{1, \mathbf{g}}(r ; \mathbf{z})\right) d r}+w(\mathbf{z}) \int_{0}^{t} \psi_{t, \varphi}(s ; \mathbf{z}) d s\right) d \mathbf{z} \\
= & \int_{Z} \frac{d}{d t}\left(f_{0}(\mathbf{z}) \varphi\left(\mathfrak{Z}_{1, \mathbf{g}}(t ; \mathbf{z})\right) \cdot \exp \left(\int_{0}^{t} u\left(\mathfrak{Z}_{1, \mathbf{g}}(r ; \mathbf{z})\right) d r\right)\right) d \mathbf{z} \\
& +\int_{Z} w(\mathbf{z}) \cdot \frac{d}{d t} \int_{0}^{t}\left(\varphi\left(\mathfrak{Z}_{1, \mathbf{g}}(t-s ; \mathbf{z})\right) \cdot e^{\int_{0}^{t-s} u\left(\mathfrak{Z}_{1, \mathbf{g}}(r ; \mathbf{z})\right) d r}\right) d s d \mathbf{z} \\
= & \int_{Z}\left\{f_{0}(\mathbf{z})\left(\nabla \varphi\left(\mathfrak{Z}_{1, \mathbf{g}}(t ; \mathbf{z})\right) \cdot \partial_{t} \mathfrak{Z}_{1, \mathbf{g}}(t ; \mathbf{z})+\varphi\left(\mathfrak{Z}_{1, \mathbf{g}}(t ; \mathbf{z})\right) u\left(\mathfrak{Z}_{1, \mathbf{g}}(t ; \mathbf{z})\right)\right) \cdot\right. \\
& +\int_{Z} w(\mathbf{z})\left\{\varphi(\mathbf{z})+\int_{0}^{t}\left(\left.\nabla \varphi\right|_{\left(\mathfrak{Z}_{1, \mathbf{g}}(t-s ; \mathbf{z})\right)} \cdot \partial_{t} \mathfrak{Z}_{1, \mathbf{g}}(t-s ; \mathbf{z})+\right.\right. \\
\stackrel{(42)}{=} & \left.\left.\int_{Z}\left(\nabla \varphi \cdot(\mathbf{v}, 0, \mathbf{g}(\mathbf{x}, \mathbf{v}, \mathbf{y}))+\left.\varphi\right|_{\left(\mathfrak{Z}_{1, \mathbf{g}}(t-s ; \mathbf{z})\right)} u\left(\mathfrak{Z}_{1, \mathbf{g}}(t-s ; \mathbf{z})\right)\right) e^{\int_{0}^{t-s} u\left(\mathfrak{Z}_{1, \mathbf{g}}(r ; \mathbf{z})\right) d r} d s\right\} d \mathbf{z}\right) \cdot f(t) d \mathbf{z}+\int_{Z} \varphi w d \mathbf{z} .
\end{aligned}
$$

Indeed, the global a priori bound on both $\|f(t)\|_{L^{2}}$ and the other functions $\mathbf{g}, u, \varphi, w$ ensure that all Lebesgue integrals here exist and so the rules of differentiation can be applied to weak derivatives. (In particular, $\varphi$ is always chosen with compact support and so we do not require additionally that $V$ is bounded.) 
5.4 Linear problem with "more regular" coefficients $\mathrm{g} \in C_{c}^{1}\left(Z, \mathbb{R}^{d}\right), u \in C^{1}(Z)$ $\cap W^{1, \infty}(Z)$ : An explicit formula for solutions in $L^{2}(Z)$

Proposition 5.18 Under the assumptions of Proposition 5.11, the weak solution $f:[0, T] \longrightarrow$ $L^{2}(Z)$ of the initial value problem (31) presented in equation (33) has the explicit form

$$
\begin{aligned}
f(t, \xi)= & f_{0}\left(\mathfrak{Z}_{-1, \mathbf{g}}(t ; \xi)\right) \cdot \exp \left(\int_{0}^{t}\left(u-\operatorname{div}_{\mathbf{y}} \mathbf{g}\right)\left(\mathfrak{Z}_{-1, \mathbf{g}}(s ; \xi)\right) d s\right)+ \\
& \int_{0}^{t}\left(w\left(\mathfrak{Z}_{-1, \mathbf{g}}(t-s ; \xi)\right) \cdot \exp \left(\int_{0}^{t-s}\left(u-\operatorname{div}_{\mathbf{y}} \mathbf{g}\right)\left(\mathfrak{Z}_{-1, \mathbf{g}}(r ; \xi)\right) d r\right)\right) d s .
\end{aligned}
$$

For our conclusions later on, the main benefit of this explicit formula is to obtain some dominating function of space and time. It will help us to verify tightness of (squared) solutions while coefficients are perturbed in a suitably bounded way (see Lemma 5.25 below).

\section{Corollary 5.19 (A function dominating this weak solution)}

Under the assumptions of Proposition 5.11, the weak solution $f:[0, T] \longrightarrow L^{2}(Z)$ of initial value problem (31) presented in equation (33) satisfies for every $t \in[0, T]$ and Lebesgue-almost every $\xi \in Z$

$$
|f(t, \xi)| \leq e^{\left(\left\|\left[\operatorname{div}_{\mathbf{y}} \mathbf{g}\right]^{-}\right\|_{L^{\infty}}+\|u\|_{L^{\infty}}\right) \cdot t} \cdot\left(\left|f_{0}\left(\mathfrak{Z}_{-1, \mathbf{g}}(t ; \xi)\right)\right|+\int_{0}^{t}\left|w\left(\mathfrak{Z}_{-1, \mathbf{g}}(t-s ; \xi)\right)\right| d s\right) .
$$

Proof of Proposition 5.18. The main idea is to change the right-hand side of equation (33) by means of the theorems of Fubini and transformations. The change of coordinates $Z \longrightarrow$ $Z, \mathbf{z} \longmapsto \mathfrak{Z}_{1, \mathbf{g}}(t ; \mathbf{z})=\xi$ has the continuously differentiable inverse $Z \longrightarrow Z, \xi \longmapsto \mathfrak{Z}_{-1, \mathbf{g}}(t ; \xi)=$ $\mathbf{z}$ and so, the transformation theorem for Lebesgue integrals leads to

$$
\begin{aligned}
& \int_{Z} \psi_{t, \varphi}(0 ; \mathbf{z}) f_{0}(\mathbf{z}) d \mathbf{z} \stackrel{(40)}{=} \int_{Z}\left\{\varphi\left(\mathfrak{Z}_{1, \mathbf{g}}(t ; \mathbf{z})\right) \cdot \exp \left(\int_{0}^{t} u\left(\mathfrak{Z}_{1, \mathbf{g}}(r ; \mathbf{z})\right) d r\right)\right\} f_{0}(\mathbf{z}) d \mathbf{z} \\
& =\int_{Z} \varphi(\xi) \cdot \exp \left(\int_{0}^{t} u\left(\mathfrak{Z}_{1, \mathbf{g}}\left(r ; \mathfrak{Z}_{-1, \mathbf{g}}(t ; \xi)\right)\right) d r\right) f_{0}\left(\mathfrak{Z}_{-1, \mathbf{g}}(t ; \xi)\right) \cdot\left|\operatorname{det} \partial_{\xi} \mathfrak{Z}_{-1, \mathbf{g}}(t ; \xi)\right| d \xi \\
& =\int_{Z} \varphi(\xi) \cdot \exp \left(\int_{0}^{t} u\left(\mathfrak{Z}_{-1, \mathbf{g}}(t-r ; \xi) d r\right) f_{0}\left(\mathfrak{Z}_{-1, \mathbf{g}}(t ; \xi)\right) \cdot\left|\operatorname{det} \partial_{\xi} \mathfrak{Z}_{-1, \mathbf{g}}(t ; \xi)\right| d \xi\right. \\
& \stackrel{(38)}{=} \int_{Z} \varphi(\xi) \cdot \exp \left(\int_{0}^{t} u\left(\mathfrak{Z}_{-1, \mathbf{g}}(t-r ; \xi) d r\right) f_{0}\left(\mathfrak{Z}_{-1, \mathbf{g}}(t ; \xi)\right) \cdot e^{-\int_{0}^{t}\left(\operatorname{div}_{\mathbf{y}} \mathbf{g}\right)\left(\mathfrak{Z}_{-1, \mathbf{g}}(s ; \xi)\right) d s} d \xi\right. \\
& =\int_{Z} \varphi(\xi) \cdot \exp \left(\int_{0}^{t}\left(u-\operatorname{div}_{\mathbf{y}} \mathbf{g}\right)\left(\mathfrak{Z}_{-1, \mathbf{g}}(s ; \xi)\right) d s\right) f_{0}\left(\mathfrak{Z}_{-1, \mathbf{g}}(t ; \xi)\right) d \xi
\end{aligned}
$$

Similarly, we obtain by means of the transformation $Z \longrightarrow Z, \mathbf{z} \longmapsto \mathfrak{Z}_{1, \mathbf{g}}(t-s ; \mathbf{z})=\xi$

$$
\begin{aligned}
& \int_{Z}\left(w(\mathbf{z}) \cdot \int_{0}^{t} \psi_{t, \varphi}(s ; \mathbf{z}) d s\right) d \mathbf{z} \\
& \stackrel{\stackrel{40}{=})}{=} \int_{0}^{t} \int_{Z} w(\mathbf{z}) \cdot \varphi\left(\mathfrak{Z}_{1, \mathbf{g}}(t-s ; \mathbf{z})\right) \cdot \exp \left(\int_{0}^{t-s} u\left(\mathfrak{Z}_{1, \mathbf{g}}(r ; \mathbf{z})\right) d r\right) d \mathbf{z} d s
\end{aligned}
$$




$$
\begin{aligned}
\int_{Z} & \left(w(\mathbf{z}) \cdot \int_{0}^{t} \psi_{t, \varphi}(s ; \mathbf{z}) d s\right) d \mathbf{z} \\
= & \int_{0}^{t} \int_{Z} w\left(\mathfrak{Z}_{-1, \mathbf{g}}(t-s ; \xi)\right) \cdot \varphi(\xi) \cdot \exp \left(\int_{0}^{t-s} u\left(\mathfrak{Z}_{1, \mathbf{g}}\left(r ; \mathfrak{Z}_{-1, \mathbf{g}}(t-s ; \xi)\right)\right) d r\right) \\
\cdot & \cdot \operatorname{det} \partial_{\xi} \mathfrak{Z}_{-1, \mathbf{g}}(t ; \xi) \mid d \xi d s \\
= & \int_{0}^{t} \int_{Z} w\left(\mathfrak{Z}_{-1, \mathbf{g}}(t-s ; \xi)\right) \cdot \varphi(\xi) \cdot \exp \left(\int_{0}^{t-s}\left(u-\operatorname{div}_{\mathbf{y}} \mathbf{g}\right)\left(\mathfrak{Z}_{-1, \mathbf{g}}(r ; \xi)\right) d r\right) d \xi d s \\
= & \int_{Z} \varphi(\xi) \cdot \int_{0}^{t}\left(w\left(\mathfrak{Z}_{-1, \mathbf{g}}(t-s ; \xi)\right) \cdot \exp \left(\int_{0}^{t-s}\left(u-\operatorname{div}_{\mathbf{y}} \mathbf{g}\right)\left(\mathfrak{Z}_{-1, \mathbf{g}}(r ; \xi)\right) d r\right)\right) d s d \xi
\end{aligned}
$$

Finally the claim results directly from equation (33).

5.5 Autonomous linear problem with "more regular" coefficients $\mathrm{g} \in C_{c}^{1}\left(Z, \mathbb{R}^{d}\right)$, $u \in C^{1}(Z) \cap W^{1, \infty}(Z) \cap L^{2}(Z):$ Further estimates

\section{Definition $\mathbf{5 . 2 0}$}

For any coefficient functions $\mathbf{g} \in C_{c}^{1}\left(Z, \mathbb{R}^{d}\right), u \in C^{1}(Z) \cap W^{1, \infty}(Z)$ and $w \in L^{2}(Z)$, let

$$
\vartheta_{\mathbf{g}, u, w}^{f}: \quad[0,1] \times L^{2}(Z) \longrightarrow L^{2}(Z), \quad\left(t, f_{0}\right) \longmapsto f(t)
$$

be defined by means of the unique weak solution of

$$
\partial_{t} f+\operatorname{div}_{\mathbf{x}}(f \mathbf{v})+\operatorname{div}_{\mathbf{y}}(f \mathbf{g})=u f+w \quad \text { in }[0, t], \quad f(0)=f_{0}
$$

whose existence and uniqueness are stated in Propositions 5.11 and 5.7 respectively.

\section{Proposition 5.21}

Suppose $\mathbf{g}, \widehat{\mathbf{g}} \in C_{c}^{1}\left(Z, \mathbb{R}^{d}\right), \quad u, \widehat{u} \in C^{1}(Z) \cap W^{1, \infty}(Z) \cap L^{2}(Z)$ and $w, \widehat{w} \in L^{2}(Z)$.

Then the following features hold for any $f_{0}, f_{1} \in L^{2}(Z)$ and $s, t \in[0,1]$ with $s+t \leq 1$ :

(1.) $\vartheta_{\mathbf{g}, u, w}^{f}\left(0, f_{0}\right)=f_{0}$

(2.) $\vartheta_{\mathbf{g}, u, w}^{f}\left(s+t, f_{0}\right)=\vartheta_{\mathbf{g}, u, w}^{f}\left(s, \vartheta_{\mathbf{g}, u, w}^{f}\left(t, f_{0}\right)\right)$

(3.) $\left\|\vartheta_{\mathbf{g}, u, w}^{f}\left(t, f_{0}\right)\right\|_{L^{2}(Z)} \leq\left(\left\|f_{0}\right\|_{L^{2}(Z)}+\|w\|_{L^{2}(Z)} t\right) \cdot e^{\operatorname{const}(n) \cdot\left(1+\left\|\left[\operatorname{div}_{\mathbf{y}} \mathbf{g}\right]^{-}\right\|_{\left.L^{\infty}+\|u\|_{L^{\infty}}\right) \cdot t}\right.}$

(4.) $\left\|\vartheta_{\mathbf{g}, u, w}^{f}\left(t, f_{0}\right)\right\|_{L^{2}(Z)} \geq\left\|f_{0}\right\|_{L^{2}(Z)} \cdot e^{-\operatorname{const}(n) \cdot\left(1+\left\|\left[\operatorname{div}_{\mathbf{y}} \mathbf{g}\right]^{+}\right\|_{L^{\infty}+\| u} \|_{\left.L^{\infty}\right) \cdot t}\right.}-\|w\|_{L^{2}(Z)} t$

(5.) $\breve{e} f\left(f_{0}, \vartheta_{\mathbf{g}, u, w}^{f}\left(t, f_{0}\right)\right) \leq t \cdot \operatorname{const}\left(n,\|\mathbf{g}\|_{L^{2}},\left\|\left[\operatorname{div}_{\mathbf{y}} \mathbf{g}\right]^{-}\right\|_{L^{\infty}},\|u\|_{L^{\infty}}, V\right) \cdot\left(\left\|f_{0}\right\|_{L^{2}}+\|w\|_{L^{2}}\right)$

(6.) $e^{f}\left(f_{0}, \vartheta_{\mathbf{g}, u, w}^{f}\left(t, f_{0}\right)\right) \leq t \cdot \operatorname{const}\left(n,\|\mathbf{g}\|_{L^{2}},\left\|\operatorname{div}_{\mathbf{y}} \mathbf{g}\right\|_{L^{\infty}},\|u\|_{L^{\infty}}, V\right) \cdot\left(\left\|f_{0}\right\|_{L^{2}}+\|w\|_{L^{2}}\right)$

(7.) $\quad d^{f}\left(\vartheta_{\mathbf{g}, u, w}^{f}\left(t, f_{0}\right), \vartheta_{\mathbf{g}, u, w}^{f}\left(t, f_{1}\right)\right) \leq d^{f}\left(f_{0}, f_{1}\right) \cdot e^{\operatorname{const}(n)\left(1+\left\|\partial_{\mathbf{y}} \mathbf{g}\right\|_{L^{\infty}}+\|u\|_{\left.L^{\infty}+\left\|\nabla_{\mathbf{y}} u\right\|_{L^{\infty}}\right) \cdot t}\right.}$

(8.) $\quad d^{f}\left(\vartheta_{\mathbf{g}, u, w}^{f}\left(t, f_{0}\right), \vartheta_{\widehat{\mathbf{g}}, \widehat{u}, \widehat{w}}^{f}\left(t, f_{0}\right)\right) \leq C \cdot\left(1+\left\|f_{0}\right\|_{L^{2}(Z)}\right) \cdot t$

$$
\left(\|\mathbf{g}-\widehat{\mathbf{g}}\|_{L^{2}(Z)}+\|u-\widehat{u}\|_{L^{2}(Z)}+\|w-\widehat{w}\|_{L^{2}(Z)}\right)
$$

with a constant $C\left(n,\left\|\partial_{\mathbf{y}} \mathbf{g}\right\|_{L^{\infty}},\left\|\partial_{\mathbf{y}} \widehat{\mathbf{g}}\right\|_{L^{\infty}},\|u\|_{L^{\infty}},\left\|\nabla_{\mathbf{y}} u\right\|_{L^{\infty}},\|\widehat{u}\|_{L^{\infty}},\left\|\nabla_{\mathbf{y}} \widehat{u}\right\|_{L^{\infty}},\|\widehat{w}\|_{L^{2}}\right)$. 
Lemma 5.22 Under the assumptions of Proposition 5.11, let $\psi_{t, \varphi} \in C^{1}([0, t] \times Z)$ denote the unique solution to problem (39) for any $t \in[0, T]$ and $\varphi \in C_{c}^{1}(Z)$ given. Correspondingly suppose $\widehat{\psi}_{t, \varphi} \in C^{1}([0, t] \times Z)$ to be the solution to problem (39) with the coefficients $\widehat{\mathrm{g}} \in$ $C_{c}^{1}\left(Z, \mathbb{R}^{d}\right)$ and $\widehat{u} \in C^{1}(Z) \cap W^{1, \infty}(Z) \cap L^{2}(Z)$ instead.

Then, for every $0 \leq s \leq t \leq T$ and $\varphi, \widehat{\varphi} \in C_{c}^{1}\left(\mathbb{R}^{n}\right)$, the following a priori estimates hold

$$
\begin{aligned}
\left\|\nabla_{\mathbf{y}} \psi_{t, \varphi}(s ; \cdot)\right\|_{L^{2}(Z)} \leq & \left(\left\|\nabla_{\mathbf{y}} \varphi\right\|_{L^{2}(Z)}+\|\varphi\|_{L^{2}(Z)}(t-s)\left\|\nabla_{\mathbf{y}} u\right\|_{L^{\infty}(Z)}\right) \cdot \\
& e^{\operatorname{const}(n) \cdot\left(1+\left\|\partial_{\mathbf{y}} \mathbf{g}\right\|_{L^{\infty}}+\|u\|_{L^{\infty}}\right) \cdot t}, \\
\left\|\psi_{t, \varphi}(s ; \cdot)-\widehat{\psi}_{t, \varphi}(s ; \cdot)\right\|_{L^{2}(Z)} \leq & \left(\|\varphi\|_{L^{\infty}}+\left\|\nabla_{\mathbf{y}} \varphi\right\|_{L^{\infty}}\right) \cdot C t\left(\|\mathbf{g}-\widehat{\mathbf{g}}\|_{L^{2}}+\|u-\widehat{u}\|_{L^{2}}\right)
\end{aligned}
$$

with a constant $C=C\left(n, T,\left\|\partial_{\mathbf{y}} \mathbf{g}\right\|_{L^{\infty}(Z)},\left\|\partial_{\mathbf{y}} \widehat{\mathbf{g}}\right\|_{L^{\infty}(Z)},\left\|\nabla_{\mathbf{y}} u\right\|_{L^{\infty}(Z)},\left\|\nabla_{\mathbf{y}} \widehat{u}\right\|_{L^{\infty}(Z)}\right)$.

Proof of Lemma 5.22. $\quad \mathfrak{Z}_{1, \mathrm{~g}}$ still denotes the solution of initial value problem (37). The explicit representation of $\psi_{t, \varphi}$, i.e. $\psi_{t, \varphi}(s ; \mathbf{z})=\varphi\left(\mathfrak{Z}_{1, \mathbf{g}}(t-s ; \mathbf{z})\right) \cdot e^{\int_{0}^{t-s} u\left(\mathfrak{Z}_{1, \mathbf{g}}(r ; \mathbf{z})\right) d r}$, provides for the gradient with respect to $\mathbf{y}$

$$
\begin{aligned}
& \left|\nabla_{\mathbf{y}} \psi_{t, \varphi}(s ; \cdot)\right| \leq\left(\left|\nabla_{\mathbf{y}} \varphi\left(\mathfrak{Z}_{1, \mathbf{g}}(t-s ; \cdot)\right) \cdot \partial_{\mathbf{y}} \mathfrak{Y}_{1, \mathbf{g}}(t-s ; \cdot)\right|+\right. \\
& \left.\left|\varphi\left(\mathfrak{Z}_{1, \mathbf{g}}(t-s ; \cdot)\right) \cdot \int_{0}^{t-s} \nabla_{\mathbf{y}} u\left(\mathfrak{Z}_{1, \mathbf{g}}(r ; \cdot)\right) \cdot \partial_{\mathbf{y}} \mathfrak{Y}_{1, \mathbf{g}}(r ; \cdot) d r\right|\right) \\
& \text {. } e^{\int_{0}^{t-s} u\left(\mathfrak{Z}_{1, \mathbf{g}}(r ; \cdot)\right) d r} \text {. }
\end{aligned}
$$

Now the transformation theorem of Lebesgue integrals leads to the following upper estimate of the first scalar product:

$$
\begin{aligned}
\int_{Z} & \left|\nabla_{\mathbf{y}} \varphi\left(\mathfrak{Z}_{1, \mathbf{g}}(t-s ; \cdot)\right) \cdot \partial_{\mathbf{y}} \mathfrak{Y}_{1, \mathbf{g}}(t-s ; \cdot)\right|^{2} d \mathbf{z} \\
& \leq\left\|\partial_{\mathbf{y}} \mathfrak{Y}_{1, \mathbf{g}}(t-s ; \cdot)\right\|_{L^{\infty}(Z)}^{2} \cdot \int_{Z}\left|\nabla_{\mathbf{y}} \varphi\left(\mathfrak{Z}_{1, \mathbf{g}}(t-s ; \mathbf{z})\right)\right|^{2} d \mathbf{z} \\
& \stackrel{(35)}{\leq} e^{\operatorname{const}(n) \cdot\left(1+\left\|\partial_{\mathbf{y}} \mathbf{g}\right\|_{L^{\infty}}\right) \cdot t} \cdot\left\|\operatorname{det}\left(\partial_{\mathbf{z}} \mathfrak{Z}_{1, \mathbf{g}}(t ; \cdot)\right)^{-1}\right\|_{L^{\infty}(Z)}\left\|\nabla_{\mathbf{y}} \varphi\right\|_{L^{2}(Z)}^{2} \\
& \stackrel{(38)}{\leq} e^{\operatorname{const}(n) \cdot\left(1+\left\|\partial_{\mathbf{y}} \mathbf{g}\right\|_{L^{\infty}}\right) \cdot t} \cdot\left\|\nabla_{\mathbf{y}} \varphi\right\|_{L^{2}(Z)}^{2} \cdot
\end{aligned}
$$

The corresponding conclusion for the second term and Minkowski inequality imply

$\left\|\nabla_{\mathbf{y}} \psi_{t, \varphi}(s ; \cdot)\right\|_{L^{2}(Z)} \leq e^{\operatorname{const}(n) \cdot\left(1+\left\|\partial_{\mathbf{y}} \mathbf{g}\right\|_{L^{\infty}}+\|u\|_{L^{\infty}}\right) \cdot t} \cdot\left(\left\|\nabla_{\mathbf{y}} \varphi\right\|_{L^{2}}+\|\varphi\|_{L^{2}}(t-s)\left\|\nabla_{\mathbf{y}} u\right\|_{L^{\infty}}\right)$.

For "comparing" the solutions $\psi_{t, \varphi}, \widehat{\psi}_{t, \varphi}$ related to the coefficients $\mathbf{g}, u$ and $\widehat{\mathbf{g}}, \widehat{u}$ respectively, we use the auxiliary functions $\mathbf{g}_{\lambda}:=\lambda \cdot \mathbf{g}+(1-\lambda) \cdot \widehat{\mathbf{g}}, u_{\lambda}:=\lambda \cdot u+(1-\lambda) \cdot \widehat{u}$ and $\Psi_{t, \varphi}^{\lambda} \in C^{1}([0, t] \times Z)$, $0 \leq \lambda \leq 1$, with

$$
\Psi_{t, \varphi}^{\lambda}(s ; \cdot):=\varphi\left(\mathfrak{Z}_{1, g_{\lambda}}(t-s ; \cdot)\right) \cdot \exp \left(\int_{0}^{t-s} u_{\lambda}\left(\mathfrak{Z}_{1, g_{\lambda}}(r ; \cdot)\right) d r\right) .
$$


In particular, $\Psi_{t, \varphi}^{1}=\psi_{t, \varphi}$ and $\Psi_{t, \varphi}^{0}=\widehat{\psi}_{t, \varphi}$. Furthermore the integral form of differential equation (37), i.e.

$$
\mathfrak{Z}_{1, g_{\lambda}}(t ; \mathbf{x}, \mathbf{v}, \mathbf{y})=(\mathbf{x}, \mathbf{v}, \mathbf{y})+\int_{0}^{t}\left(\mathbf{v}, 0, \mathbf{g}_{\lambda}\left(\mathfrak{Z}_{1, g_{\lambda}}(s ; \mathbf{x}, \mathbf{v}, \mathbf{y})\right)\right) d s,
$$

provides an integral equation for the partial derivative $\mathfrak{Z}_{1, g_{\lambda}}(\cdot ; \mathbf{z})$ w.r.t. parameter $\lambda$ :

$$
\begin{aligned}
\partial_{\lambda} \mathfrak{Z}_{1, g_{\lambda}}(t ; \mathbf{z}) & =\int_{0}^{t}\left(0,0,(\mathbf{g}-\widehat{\mathbf{g}})\left(\mathfrak{Z}_{1, g_{\lambda}}(s ; \mathbf{z})\right)+D \mathbf{g}_{\lambda}\left(\mathfrak{Z}_{1, g_{\lambda}}(s ; \mathbf{z})\right) \cdot \partial_{\lambda} \mathfrak{Z}_{1, g_{\lambda}}(s ; \mathbf{z})\right) d s \\
& =\int_{0}^{t}\left(0,0,(\mathbf{g}-\widehat{\mathbf{g}})\left(\mathfrak{Z}_{1, g_{\lambda}}(s ; \mathbf{z})\right)+\partial_{\mathbf{y}} \mathbf{g}_{\lambda}\left(\mathfrak{Z}_{1, g_{\lambda}}(s ; \mathbf{z})\right) \cdot \partial_{\lambda} \mathfrak{Y}_{1, g_{\lambda}}(s ; \mathbf{z})\right) d s \\
\left|\partial_{\lambda} \mathfrak{Z}_{1, g_{\lambda}}(t ; \mathbf{z})\right|^{2} & \leq t \cdot \int_{0}^{t}\left|(\mathbf{g}-\widehat{\mathbf{g}})\left(\mathfrak{Z}_{1, g_{\lambda}}(s ; \mathbf{z})\right)+\partial_{\mathbf{y}} \mathbf{g}_{\lambda}\left(\mathfrak{Z}_{1, g_{\lambda}}(s ; \mathbf{z})\right) \cdot \partial_{\lambda} \mathfrak{Y}_{1, g_{\lambda}}(s ; \mathbf{z})\right|^{2} d s \\
& \leq 2 t \cdot \int_{0}^{t}\left(\left|(\mathbf{g}-\widehat{\mathbf{g}})\left(\mathfrak{Z}_{1, g_{\lambda}}(s ; \mathbf{z})\right)\right|^{2}+\left\|\partial_{\mathbf{y}} \mathbf{g}_{\lambda}\right\|_{L^{\infty}}^{2}\left|\partial_{\lambda} \mathfrak{Y}_{1, g_{\lambda}}(s ; \mathbf{z})\right|^{2}\right) d s \\
\left\|\partial_{\lambda} \mathfrak{Z}_{1, g_{\lambda}}(t ; \cdot)\right\|_{L^{2}}^{2} & \leq 2 t \cdot \int_{0}^{t}\left(\|\mathbf{g}-\widehat{\mathbf{g}}\|_{L^{2}(Z)}^{2} \cdot e^{c s}+\left\|\partial_{\mathbf{y}} \mathbf{g}_{\lambda}\right\|_{L^{\infty}}^{2}\left\|\partial_{\lambda} \mathfrak{Y}_{1, g_{\lambda}}(s ; \mathbf{z})\right\|_{L^{2}}^{2}\right) d s \\
& =2 t \cdot \int_{0}^{t}\left(\|\mathbf{g}-\widehat{\mathbf{g}}\|_{L^{2}(Z)}^{2} \cdot e^{c s}+\left\|\partial_{\mathbf{y}} \mathbf{g}_{\lambda}\right\|_{L^{\infty}}^{2}\left\|\partial_{\lambda} \mathfrak{Z}_{1, g_{\lambda}}(s ; \mathbf{z})\right\|_{L^{2}}^{2}\right) d s
\end{aligned}
$$

with a constant $c=c\left(n,\left\|\left[\operatorname{div}_{\mathbf{y}} \mathbf{g}\right]^{-}\right\|_{L^{\infty}},\left\|\left[\operatorname{div}_{\mathbf{y}} \widehat{\mathbf{g}}\right]^{-}\right\|_{L^{\infty}}\right)$ and, due to Gronwall's inequality,

$$
\left\|\partial_{\lambda} \mathfrak{Y}_{1, g_{\lambda}}(t ; \cdot)\right\|_{L^{2}}=\left\|\partial_{\lambda} \mathfrak{Z}_{1, g_{\lambda}}(t ; \cdot)\right\|_{L^{2}} \leq\|\mathbf{g}-\widehat{\mathbf{g}}\|_{L^{2}} \cdot t e^{t \cdot \operatorname{const}\left(n, T,\left\|\partial_{\mathbf{y}} \mathbf{g}\right\|_{L^{\infty}},\left\|\partial_{\mathbf{y}} \widehat{\mathbf{g}}\right\|_{L^{\infty}}\right)} .
$$

Hence,

$$
\begin{aligned}
\partial_{\lambda} \Psi_{t, \varphi}^{\lambda}(s ; \mathbf{z})= & e^{\int_{0}^{t-s} u_{\lambda}\left(\mathfrak{Z}_{1, g_{\lambda}}(r ; \mathbf{z})\right) d r} \cdot\left\{\left.\partial_{\mathbf{y}} \varphi\right|_{\mathfrak{Z}_{1, g_{\lambda}}(t-s ; \mathbf{z})} \cdot \partial_{\lambda} \mathfrak{Y}_{1, g_{\lambda}}(t-s ; \mathbf{z})+\right. \\
& \left.\left.\varphi\right|_{\mathfrak{Z}_{1, g_{\lambda}}(t-s ; \mathbf{z})} \cdot \int_{0}^{t-s}\left(\left.(u-\widehat{u})\right|_{\mathfrak{Z}_{1, g_{\lambda}}(r ; \mathbf{z})}+\left.\partial_{\mathbf{y}} u_{\lambda}\right|_{\mathfrak{Z}_{1, g_{\lambda}}(r ; \mathbf{z})} \cdot \partial_{\lambda} \mathfrak{Y}_{1, g_{\lambda}}(r ; \mathbf{z})\right) d r\right\}
\end{aligned}
$$

implies the upper estimate

$$
\begin{aligned}
\| \partial_{\lambda} & \Psi_{t, \varphi}^{\lambda}(s ; \cdot) \|_{L^{2}(Z)} \\
\leq & e^{(t-s) \cdot\left(\|u\|_{L^{\infty}}+\|\widehat{u}\|_{L^{\infty}}\right)} \cdot\left\{\left\|\partial_{\mathbf{y}} \varphi\left(\mathfrak{Z}_{1, g_{\lambda}}(t-s ; \cdot)\right)\right\|_{L^{\infty}(Z)} \cdot\left\|\partial_{\lambda} \mathfrak{Y}_{1, g_{\lambda}}(t-s ; \cdot)\right\|_{L^{2}(Z)}+\right. \\
& \left.\left.\|\varphi\|_{L^{\infty}(Z)} \cdot(t-s)\left(\|u-\widehat{u}\|_{L^{2}} \cdot e^{c(t-s)}+\left\|\partial_{\mathbf{y}} u_{\lambda}\right\|_{L^{\infty}} \cdot \sup _{r \leq t-s} \| \partial_{\lambda} \mathfrak{Y}\right)_{1, g_{\lambda}}(r ; \cdot) \|_{L^{2}(Z)}\right)\right\} \\
\leq & e^{t \cdot \operatorname{const}\left(n, T,\left\|\partial_{\mathbf{y}} \mathbf{g}\right\|_{L^{\infty}},\left\|\partial_{\mathbf{y}} \widehat{\widehat{g}}\right\|_{L^{\infty}},\|u\|_{L^{\infty}},\|\widehat{u}\|_{L^{\infty}}\right)} \cdot(t-s) \cdot\left\{\left\|\partial_{\mathbf{y}} \varphi\right\|_{L^{\infty}(Z)}\|\mathbf{g}-\widehat{\mathbf{g}}\|_{L^{2}(Z)}+\right. \\
& \left.\|\varphi\|_{L^{\infty}(Z)}\left(\|u-\widehat{u}\|_{L^{2}(Z)}+\left(\left\|\partial_{\mathbf{y}} u\right\|_{L^{\infty}}+\left\|\partial_{\mathbf{y}} \widehat{u}\right\|_{L^{\infty}}\right) \cdot\|\mathbf{g}-\widehat{\mathbf{g}}\|_{L^{2}(Z)}(t-s)\right)\right\} .
\end{aligned}
$$

The last claimed inequality now results from

$$
\left\|\psi_{t, \varphi}(s ; \cdot)-\widehat{\psi}_{t, \varphi}(s ; \cdot)\right\|_{L^{2}(Z)} \leq \int_{0}^{1}\left\|\partial_{\lambda} \Psi_{t, \varphi}^{\lambda}(s ; \cdot)\right\|_{L^{2}(Z)} d \lambda .
$$




\section{Proof of Proposition 5.21.}

(1.) The property $\vartheta_{\mathbf{g}, u, w}^{f}\left(0, f_{0}\right)=f_{0}$ results immediately from Definition 5.20 of $\vartheta_{\mathbf{g}, u, w}^{f}$.

(2.) The semigroup property $\vartheta_{\mathbf{g}, u, w}^{f}\left(s+t, f_{0}\right)=\vartheta_{\mathbf{g}, u, w}^{f}\left(s, \vartheta_{\mathbf{g}, u, w}^{f}\left(t, f_{0}\right)\right)$ is a consequence of the uniqueness of solutions to the initial value problem (31) as stated in Proposition 5.7.

(3.) This upper $L^{2}$ bound has already been formulated (and proved) in Proposition 5.11.

(4.) Choose any $t \in[0,1]$ and $f_{0} \in L^{2}(Z)$. The characterising condition (32) on weak solutions implies immediately: $f:[0, t] \longrightarrow L^{2}(Z)$ is a weak solution of initial value problem (32) if and only if $\widehat{f}:=f(t-\cdot):[0, t] \longrightarrow L^{2}(Z)$ is a weak solution of

$$
\partial_{t} \widehat{f}-\operatorname{div}_{\mathbf{x}}(\widehat{f} \mathbf{v})-\operatorname{div}_{\mathbf{y}}(\widehat{f} \mathbf{g})=-u \widehat{f}-w \quad \text { in }[0, t], \quad \widehat{f}(0)=f(t) .
$$

Due to the uniqueness mentioned in Proposition 5.7, we conclude

$$
\vartheta_{\mathbf{g}, u, w}^{f}(t, \cdot)^{-1}=\vartheta_{-\mathbf{g},-u,-w}^{f}(t, \cdot): L^{2}(Z) \longrightarrow L^{2}(Z) .
$$

Now the claimed lower $L^{2}$ bound of $f(t):=\vartheta_{\mathbf{g}, u, w}^{f}\left(t, f_{0}\right)$ results from property (3.):

$$
\begin{aligned}
\left\|f_{0}\right\|_{L^{2}} & =\left\|\vartheta_{-\mathbf{g},-u,-w}^{f}(t, f(t))\right\|_{L^{2}} \\
& \leq\left(\|f(t)\|_{L^{2}(Z)}+\|w\|_{L^{2}(Z)} t\right) \cdot e^{\operatorname{const}(n) \cdot\left(1+\left\|\left[-\operatorname{div}_{\mathbf{y}} \mathbf{g}\right]^{-}\right\|_{L^{\infty}}+\|u\|_{L^{\infty}}\right) \cdot t} .
\end{aligned}
$$

(5.) For $f(t):=\vartheta_{\mathbf{g}, u, w}^{f}\left(t, f_{0}\right), t \in[0,1]$ and $\varphi \in C_{c}^{1}(Z)$, equation (32) and property (3.) imply

$$
\begin{aligned}
& \int_{Z} \varphi\left(f(t)-f_{0}\right) d \mathbf{z} \\
& =\int_{0}^{t} \int_{Z}\left(f(s, \cdot)\left(\mathbf{v} \cdot \nabla_{\mathbf{x}} \varphi+\mathbf{g} \cdot \nabla_{\mathbf{y}} \varphi+u \varphi\right)\right) d \mathbf{z} d s \\
& \leq \int_{0}^{t}\left(\|f(s)\|_{L^{2}}\left(\|\mathbf{g}\|_{L^{2}}\|\nabla \varphi\|_{L^{\infty}}+\left(\operatorname{const}(V)+\|u\|_{L^{\infty}}\right)\|\varphi\|_{W^{1,2}}\right)\right) d s+t \cdot\|\varphi\|_{L^{2}}\|w\|_{L^{2}} \\
& \leq t \cdot\left(\|\varphi\|_{W^{1,2}(Z)}+\|\varphi\|_{W^{1, \infty}(Z)}\right) \cdot\left(\left\|f_{0}\right\|_{L^{2}(Z)}+\|w\|_{L^{2}(Z)}\right)\left(\operatorname{const}(V)+\|\mathbf{g}\|_{L^{2}}+\|u\|_{L^{\infty}}\right) \\
& \cdot \exp \left(\operatorname{const}(n) \cdot\left(1+\left\|\left[\operatorname{div}_{\mathbf{y}} \mathbf{g}\right]^{-}\right\|_{L^{\infty}}+\|u\|_{L^{\infty}}\right)\right) .
\end{aligned}
$$

The supremum w.r.t. all $\varphi \in C_{c}^{1}(Z)$ satisfying both $\|\varphi\|_{W^{1,2}(Z)} \leq 1$ and $\|\varphi\|_{W^{1, \infty}(Z)} \leq 1$ leads to the claimed estimate of $\breve{e} f\left(f_{0}, f(t)\right)$.

(6.) Statements (3.), (4.) imply the right continuity of $[0,1] \longrightarrow \mathbb{R}, t \longmapsto\left\|\vartheta_{\mathbf{g}, u, w}^{f}\left(t, f_{0}\right)\right\|_{L^{2}}$ at $t=0$. Due to the semigroup property (2.), the norm is right continuous at every $t \in[0,1[$. By means of (44), we similarly obtain the left continuity of $\left\|\vartheta_{\mathbf{g}, u, w}^{f}\left(\cdot, f_{0}\right)\right\|_{L^{2}}$ in $\left.] 0,1\right]$.

Furthermore statements (3.), (4.) guarantee that the right Dini derivative is absolutely bounded at each time instant $t \in[0,1[$ :

$$
\begin{aligned}
\limsup _{h \downarrow 0} & \left|\frac{\left\|\vartheta_{\mathbf{g}, u, w}^{f}\left(t+h, f_{0}\right)\right\|_{L^{2}}-\left\|\vartheta_{\mathbf{g}, u, w}^{f}\left(t, f_{0}\right)\right\|_{L^{2}}}{h}\right| \\
& \leq \operatorname{const}\left(n,\left\|\operatorname{div}_{\mathbf{y}} \mathbf{g}\right\|_{L^{\infty}},\|u\|_{L^{\infty}}\right) \cdot\left(\left\|\vartheta_{\mathbf{g}, u, w}^{f}\left(t, f_{0}\right)\right\|_{L^{2}}+\|w\|_{L^{2}}\right) \\
& \stackrel{(3 .)}{\leq} \operatorname{const}\left(n,\left\|\operatorname{div}_{\mathbf{y}} \mathbf{g}\right\|_{L^{\infty}},\|u\|_{L^{\infty}}\right) \cdot\left(\left\|f_{0}\right\|_{L^{2}}+\|w\|_{L^{2}}\right)
\end{aligned}
$$


and so, $\left\|\vartheta_{\mathbf{g}, u, w}^{f}\left(\cdot, f_{0}\right)\right\|_{L^{2}}:[0,1] \longrightarrow[0, \infty[$ is even Lipschitz continuous. Together with property (5.), it leads to the claimed Lipschitz continuity of $\vartheta_{\mathbf{g}, u, w}^{f}\left(\cdot, f_{0}\right)$ w.r.t. $e^{f}$.

(7.) For every test function $\varphi \in C_{c}^{1}(Z)$, the representations (33), (40) guarantee for the solutions $\vartheta_{\mathbf{g}, u, w}^{f}\left(t, f_{0}\right)$ and $\vartheta_{\mathbf{g}, u, w}^{f}\left(t, f_{1}\right)$ related to initial states $f_{0}, f_{1} \in L^{2}(Z)$ respectively

$$
\begin{aligned}
\int_{Z} \varphi & \cdot\left(\vartheta_{\mathbf{g}, u, w}^{f}\left(t, f_{0}\right)-\vartheta_{\mathbf{g}, u, w}^{f}\left(t, f_{1}\right)\right) d \mathbf{z}=\int_{Z} \psi_{t, \varphi}(0 ; \cdot)\left(f_{0}-f_{1}\right) d \mathbf{z} \\
& =\int_{Z} \varphi\left(\mathfrak{Z}_{1, \mathbf{g}}(t ; \cdot)\right) \cdot \exp \left(\int_{0}^{t} u\left(\mathfrak{Z}_{1, \mathbf{g}}(r ; \cdot)\right) d r\right)\left(f_{0}-f_{1}\right) d \mathbf{z} .
\end{aligned}
$$

By means of density arguments, this relation can be extended to any auxiliary function $\varphi \in$ $C_{c}^{0}(Z)$ with $\partial_{\mathbf{y}} \varphi \in L^{\infty}(Z)$. Due to the assumptions $g \in C_{c}^{1}\left(Z, \mathbb{R}^{n}\right), u \in C^{1}(Z)$, the function

$$
Z \longrightarrow \mathbb{R}, \quad \mathbf{z} \longmapsto \varphi\left(\mathfrak{Z}_{1, \mathbf{g}}(t ; \mathbf{z})\right) \cdot \exp \left(\int_{0}^{t} u\left(\mathfrak{Z}_{1, \mathbf{g}}(r ; \mathbf{z})\right) d r\right)
$$

is continuous with compact support in $Z$ at each time instant $t \in[0,1]$. Moreover for every $\mathbf{x} \in \mathbb{R}^{n}, \mathbf{v} \in V$ fixed arbitrarily, the composition $Y \longrightarrow \mathbb{R}, \mathbf{y} \longmapsto \varphi\left(\mathfrak{Z}_{1, \mathbf{g}}(t ; \mathbf{x}, \mathbf{v}, \mathbf{y})\right)$ also belongs to the Sobolev space $W^{1, \infty}(Y)$ and, the following a priori estimates hold

$$
\begin{aligned}
& \left\|\varphi\left(\mathfrak{Z}_{1, \mathbf{g}}(t ; \cdot)\right)\right\|_{L^{2}(Z)}^{2} \leq\|\varphi\|_{L^{2}(Z)}^{2} \cdot\left\|\operatorname{det}\left(\partial_{\mathbf{z}} \mathfrak{Z}_{1, \mathbf{g}}(t ; \cdot)\right)^{-1}\right\|_{L^{\infty}(Z)} \\
& \stackrel{(38)}{\leq}\|\varphi\|_{L^{2}(Z)}^{2} \cdot e^{\operatorname{const}(n) \cdot\left\|\left[\operatorname{div}_{\mathbf{y}} \mathbf{g}\right]^{-}\right\|_{L^{\infty}(Z)} \cdot t}, \\
& \left\|\partial_{\mathbf{y}}\left(\varphi \circ \mathfrak{Z}_{1, \mathbf{g}}(t ; \cdot)\right)\right\|_{L^{\infty}(Z)} \leq\left\|\partial_{\mathbf{y}} \varphi\right\|_{L^{\infty}(Z)} \cdot\left\|\partial_{\mathbf{y}} \mathfrak{Y}_{1, \mathbf{g}}(t ; \cdot)\right\|_{L^{\infty}(Z)} \\
& \stackrel{(35)}{\leq}\left\|\partial_{\mathbf{y}} \varphi\right\|_{L^{\infty}(Z)} \cdot e^{\operatorname{const}(n) \cdot\left(1+\left\|\partial_{\mathbf{y}} \mathbf{g}\right\|_{L^{\infty}(Z)}\right) \cdot t} .
\end{aligned}
$$

Correspondingly, the second factor in representation (45) of the difference, i.e. the composition

$$
\eta_{\mathbf{g}, u, t}: Z \longrightarrow \mathbb{R}, \quad \mathbf{z} \longmapsto \exp \left(\int_{0}^{t} u\left(\mathfrak{Z}_{1, \mathbf{g}}(r ; \cdot)\right) d r\right),
$$

and its partial derivative w.r.t. $\mathbf{y}$ belong to $L^{\infty}(Z)$ with the a priori bound

$$
\begin{aligned}
\left\|\partial_{\mathbf{y}} \eta_{\mathbf{g}, u, t}\right\|_{L^{\infty}(Z)} & \leq e^{\|u\|_{L^{\infty}(Z)} t} \cdot \int_{0}^{t}\left\|\partial_{\mathbf{y}} u\left(\mathfrak{Z}_{1, \mathbf{g}}(r ; \cdot)\right)\right\|_{L^{\infty}(Z)}\left\|\partial_{\mathbf{y}} \mathfrak{Y}_{1, \mathbf{g}}(r ; \cdot)\right\|_{L^{\infty}(Z)} d r \\
& \stackrel{(35)}{\leq} e^{\operatorname{const}(n)\left(1+\left\|\partial_{\mathbf{y}} \mathbf{g}\right\|_{L^{\infty}(Z)}+\|u\|_{L^{\infty}(Z)}\right) t} \cdot\left\|\partial_{\mathbf{y}} u\right\|_{L^{\infty}(Z)} t .
\end{aligned}
$$

Hence, standard arguments (about homogeneity w.r.t. the norm of test functions) lead to

$$
\begin{aligned}
& d^{f}\left(\vartheta_{\mathbf{g}, u, w}^{f}\left(t, f_{0}\right), \vartheta_{\mathbf{g}, u, w}^{f}\left(t, f_{1}\right)\right) \\
& \stackrel{(45)}{=} \sup \left\{\int_{Z} \varphi\left(\mathfrak{Z}_{1, \mathbf{g}}(t ; \cdot)\right) \eta_{\mathbf{g}, u, t} \cdot\left(f_{0}-f_{1}\right) \quad d \mathbf{z} \mid \varphi \in C_{c}^{0}(Z), \partial_{\mathbf{y}} \varphi \in L^{\infty}(Z),\right. \\
& \left.\|\varphi\|_{L^{2}} \leq 1,\|\varphi\|_{L^{\infty}} \leq 1,\left\|\partial_{\mathbf{y}} \varphi\right\|_{L^{\infty}} \leq 1\right\} \\
& \leq e^{\operatorname{const}(n)\left(1+\left\|\partial_{\mathbf{y}} \mathbf{g}\right\|_{L^{\infty}(Z)}+\|u\|_{L^{\infty}(Z)}+\left\|\partial_{\mathbf{y}} u\right\|_{L^{\infty}(Z)}\right) \cdot t} \cdot d^{f}\left(f_{0}, f_{1}\right) .
\end{aligned}
$$


(8.) For every test function $\varphi \in C_{c}^{1}(Z)$, let $\psi_{t, \varphi}, \widehat{\psi}_{t, \varphi} \in C^{1}([0, t] \times Z)$ denote the auxiliary functions as specified in Lemma 5.22. We conclude from the estimates in Lemmas 5.17, 5.22

$$
\begin{aligned}
& \int_{Z} \varphi \cdot\left(\vartheta_{\mathbf{g}, u, w}^{f}\left(t, f_{0}\right)-\vartheta_{\widehat{\mathbf{g}}, \widehat{u}, \widehat{w}}^{f}\left(t, f_{0}\right)\right) d \mathbf{z} \\
& \stackrel{(33)}{=} \int_{Z}\left(\left(\psi_{t, \varphi}(0 ; \cdot)-\widehat{\psi}_{t, \varphi}(0 ; \cdot)\right) f_{0}+w \int_{0}^{t} \psi_{t, \varphi}(s ; \cdot) d s-\widehat{w} \int_{0}^{t} \widehat{\psi}_{t, \varphi}(s ; \cdot) d s\right) d \mathbf{z} \\
& \leq\left\|\psi_{t, \varphi}(0 ; \cdot)-\widehat{\psi}_{t, \varphi}(0 ; \cdot)\right\|_{L^{2}(Z)}\left\|f_{0}\right\|_{L^{2}(Z)}+\|w-\widehat{w}\|_{L^{2}(Z)} \cdot \sup _{s \leq t}\left\|\psi_{t, \varphi}(s ; \cdot)\right\|_{L^{2}(Z)} t \\
& \quad+\|\widehat{w}\|_{L^{2}(Z)} \cdot \sup _{s \leq t}\left\|\psi_{t, \varphi}(s ; \cdot)-\widehat{\psi}_{t, \varphi}(s ; \cdot)\right\|_{L^{2}(Z)} t \\
& \quad C \cdot\left(\|\varphi\|_{L^{\infty}}+\| \partial_{\left.\mathbf{y} \varphi \|_{L^{\infty}}\right)}\left(1+\left\|f_{0}\right\|_{L^{2}(Z)}\right)\right. \\
& \quad+\|\varphi\|_{L^{2}(Z)} \cdot e^{\operatorname{const}(n) \cdot\left(1+\left\|\left[\operatorname{div}_{\mathbf{y}} \mathbf{g}\right]^{-}\right\|_{\left.L^{\infty}+\|u\|_{L^{\infty}}\right) \cdot t} \cdot t \quad\|w-\widehat{\mathbf{g}}\|_{L^{2}}+\|u-\widehat{u}\|_{L^{2}}\right)}
\end{aligned}
$$

with a constant $C=C\left(n, T,\left\|\partial_{\mathbf{y}} \mathbf{g}\right\|_{L^{\infty}(Z)},\left\|\partial_{\mathbf{y}} \widehat{\mathbf{g}}\right\|_{L^{\infty}(Z)},\left\|\partial_{\mathbf{y}} u\right\|_{L^{\infty}(Z)},\left\|\partial_{\mathbf{y}} \widehat{u}\right\|_{L^{\infty}(Z)},\|\widehat{w}\|_{L^{2}(Z)}\right)$.

\subsection{Extending existence and estimates for autonomous linear problems to "less regular" coefficients: $\mathrm{g} \in L^{\infty}\left(Z, \mathbb{R}^{d}\right) \cap L^{2}, \partial_{\mathbf{y}} \mathbf{g} \in L^{\infty}, u \in L^{\infty}(Z) \cap L^{2}$, $\partial_{\mathbf{y}} u \in L^{\infty}\left(Z, \mathbb{R}^{d}\right), w \in L^{2}(Z)$}

In comparison with Proposition 5.11 about existence of solutions, the coefficients $\mathbf{g}, u, w$ are now supposed to satisfy the weaker regularity assumptions of main Theorem 3.2, but we still consider the autonomous problem:

\section{Proposition 5.23}

Assume $\mathbf{g} \in L^{\infty}\left(Z, \mathbb{R}^{d}\right) \cap L^{2}, \partial_{\mathbf{y}} \mathbf{g} \in L^{\infty}\left(Z, \mathbb{R}^{d \times d}\right), u \in L^{\infty}(Z) \cap L^{2}, \partial_{\mathbf{y}} u \in L^{\infty}\left(Z, \mathbb{R}^{d}\right), w \in L^{2}(Z)$. Then for any initial function $f_{0} \in L^{2}(Z)$, there exists a weak solution $f:[0, T] \longrightarrow L^{2}(Z)$ of the following initial value problem in the sense of Proposition 5.11

$$
\partial_{t} f+\operatorname{div}_{\mathbf{x}}(f \mathbf{v})+\operatorname{div}_{\mathbf{y}}(f \mathbf{g})=u f+w \quad \text { in }[0,1], \quad f(0)=f_{0} .
$$

Furthermore this solution is unique and, all estimates in Proposition 5.21 hold under these weaker regularity assumptions.

The proof is essentially based on smoothing the coefficients $\mathbf{g}, u$ such that Proposition 5.11 can be applied without any significant effects on the a priori estimates.

The following lemma can be verified quite easily by means of mollifying with some Dirac sequence of smooth functions (see, e.g., [38, $\S$ VIII.3]) - in combination with any cut-off function for the compact support. It is worth mentioning that this lemma states just the $L^{2}$ convergence of the sequence $\left(\mathbf{g}_{k}\right)_{k \in \mathbb{N}}$, but not the $L^{\infty}$ convergence of the derivatives $\left(\partial_{\mathbf{y}} \mathbf{g}_{k}\right)_{k \in \mathbb{N}}$. 
Lemma 5.24 $C^{\infty}\left(Z, \mathbb{R}^{m}\right) \cap L^{2}\left(Z, \mathbb{R}^{m}\right)$ is dense in $\left\{\mathbf{h} \in L^{2}\left(Z, \mathbb{R}^{m}\right) \mid \partial_{\mathbf{y}} \mathbf{h} \in L^{\infty}\left(Z, \mathbb{R}^{m \times d}\right)\right\}$ w.r.t. $L^{2}$. In particular, for every function $\mathbf{h} \in L^{2}\left(Z, \mathbb{R}^{m}\right)$ with distributional derivative $\partial_{\mathbf{y}} \mathbf{h} \in$ $L^{\infty}\left(Z, \mathbb{R}^{m \times d}\right)$, there exists a sequence $\left(\mathbf{h}_{k}\right)_{k \in \mathbb{N}}$ in $C_{c}^{\infty}\left(Z, \mathbb{R}^{m}\right)$ with

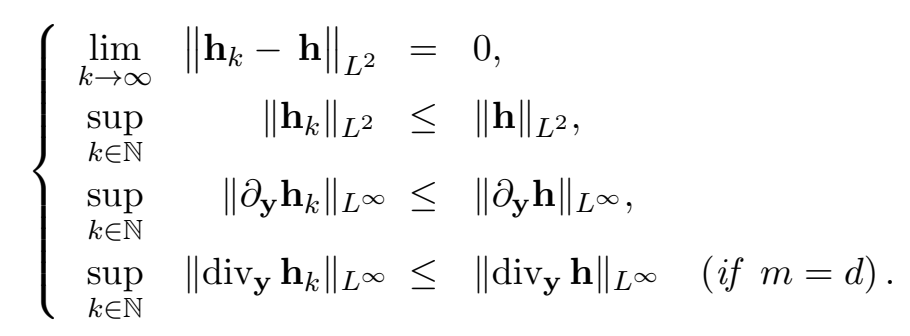

Moreover for any function $\mathbf{h} \in L^{\infty}\left(Z, \mathbb{R}^{m}\right) \cap L^{2}\left(Z, \mathbb{R}^{m}\right)$, the sequence $\left(\mathbf{h}_{k}\right)_{k \in \mathbb{N}}$ in $C_{c}^{\infty}\left(Z, \mathbb{R}^{m}\right)$ can be constructed in such a way that

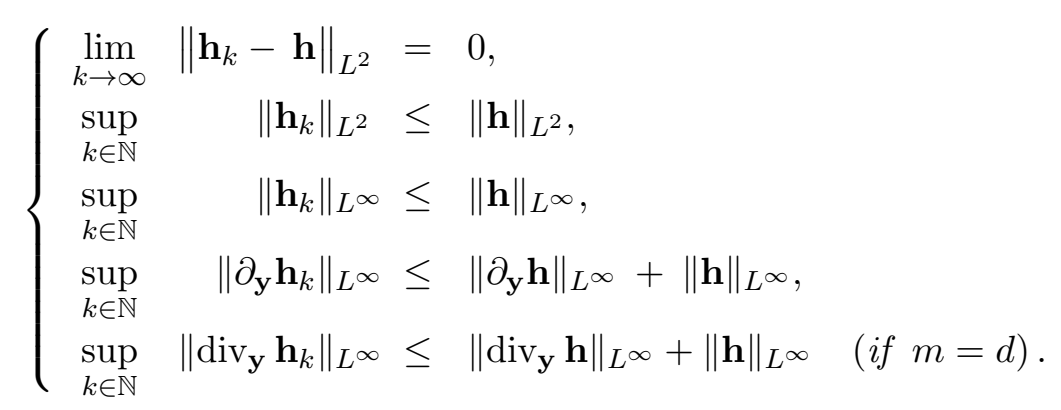

In general any proof via smoothing coefficients requires some limit process in the end and so, we need completeness of the underlying subset of $L^{2}(Z)$ with respect to $d^{f}$. As a consequence of Lemma 5.6, this feature is closely related to tightness of the square values (of solutions to the approximate problems). The dominating function mentioned in Corollary 5.19 provides the tool for specifying which norms of the coefficients should be uniformly bounded in this context:

\section{Lemma 5.25 (Tight values due to uniformly bounded velocities)}

Let $G \subseteq C_{c}^{1}\left(Z, \mathbb{R}^{d}\right)$ and $U \subseteq C^{1}(Z) \cap W^{1, \infty}(Z)$ be any nonempty subsets with

$$
\gamma:=\sup \left\{\|\mathbf{g}\|_{L^{\infty}}+\left\|\operatorname{div}_{\mathbf{y}} \mathbf{g}\right\|_{L^{\infty}}+\|u\|_{L^{\infty}} \mid \mathbf{g} \in G, u \in U\right\}<\infty .
$$

Suppose $W:=\left\{w \in L^{2}(Z)|| w \mid \leq \widehat{w}\right.$ Lebesgue-a.e. in $\left.Z \backslash\left(K_{\mathbf{x}} \times V \times K_{\mathbf{y}}\right)\right\}$ for some $\widehat{w} \in L^{2}(Z)$ and compact sets $K_{\mathbf{x}} \subseteq \mathbb{R}^{n}, K_{\mathbf{y}} \subseteq Y=\mathbb{R}^{d}$.

Then for every $f_{0} \in L^{2}(Z)$, the subset $\left\{\left|\vartheta_{\mathbf{g}, u, w}^{f}\left(t, f_{0}\right)\right|^{2} \mid t \in[0,1], \mathbf{g} \in G, u \in U, w \in W\right\} \subseteq$ $L^{1}(Z)$ is tight.

Proof of Lemma 5.25. It is based on the simple observation that $\left|\mathfrak{Z}_{1, \mathbf{g}}(t, \mathbf{z})-\mathbf{z}\right| \leq C t$ holds for all $\mathbf{z} \in Z, t \geq 0$ with the constant $C:=\sup _{\mathbf{v} \in V}|\mathbf{v}|+\sup _{\mathbf{g} \in G}\|\mathbf{g}\|_{L^{\infty}}<\infty$.

Indeed, for every $\varepsilon>0$, there exists a radius $\rho>0$ with

$$
\left\|f_{0}\right\|_{L^{2}\left(Z \backslash\left(\mathbb{B}_{\rho}\left(K_{\mathbf{x}}\right) \times V \times \mathbb{B}_{\rho}\left(K_{\mathbf{y}}\right)\right)\right)}+\|\widehat{w}\|_{L^{2}\left(Z \backslash\left(\mathbb{B}_{\rho}\left(K_{\mathbf{x}}\right) \times V \times \mathbb{B}_{\rho}\left(K_{\mathbf{y}}\right)\right)\right)}<\varepsilon .
$$


Set $R:=\rho+C$ and $Z_{r}:=Z \backslash\left(\mathbb{B}_{r}\left(K_{\mathbf{x}}\right) \times V \times \mathbb{B}_{r}\left(K_{\mathbf{y}}\right)\right) \subseteq Z$ for each $r \geq 0$. Due to Corollary 5.19, the function $\xi_{t}:=\vartheta_{\mathbf{g}, u, w}^{f}\left(t, f_{0}\right) \in L^{2}(Z)$ with any $\mathbf{g} \in G, u \in U, w \in W, t \in[0,1]$ satisfies

$$
\begin{aligned}
& \left\|\xi_{t}\right\|_{L^{2}\left(Z_{R}\right)}^{2}=\int_{Z_{R}}\left|\xi_{t}(\mathbf{z})\right|^{2} d \mathbf{z} \\
& \leq 2 \cdot e^{2\left(\left\|\operatorname{div}_{\mathbf{y}} \mathbf{g}\right\|_{L^{\infty}}+\|u\|_{L^{\infty}}\right) \cdot t}\left(\int_{Z_{R}}\left|f_{0}\left(\mathfrak{Z}_{-1, \mathbf{g}}(t, \mathbf{z})\right)\right|^{2} d \mathbf{z}+\int_{Z_{R}} \sup _{s \in[0, t]}\left|w\left(\mathfrak{Z}_{-1, \mathbf{g}}(s, \mathbf{z})\right)\right|^{2} d \mathbf{z}\right) \\
& \leq 2 \cdot e^{2\left(2\left\|\operatorname{div}_{\mathbf{y}} \mathbf{g}\right\|_{L^{\infty}}+\|u\|_{L^{\infty}}\right) \cdot t} \int_{Z_{\rho}}\left(\left|f_{0}\right|^{2}+|\widehat{w}|^{2}\right) d \mathbf{z}
\end{aligned}
$$

because for each $k \in \mathbb{N}$ and $s \in[0,1]$, the diffeomorphism $\mathfrak{Z}_{-1, \mathbf{g}}(s, \cdot): Z \longrightarrow Z$ maps $Z_{R}$ in a subset of $Z_{\rho}$. Hence we obtain $\left\|\xi_{t}\right\|_{L^{2}\left(Z_{R}\right)}^{2} \leq \operatorname{const}(\gamma) \cdot \varepsilon$ with a constant depending on the fixed bound $\gamma<\infty$ (but not on $\mathbf{g}, u, w$ or $t \in[0,1]$ ).

\section{Proof of Proposition 5.23.}

Choose sequences $\left(\mathbf{g}_{k}\right)_{k \in \mathbb{N}}$ in $C_{c}^{\infty}\left(Z, \mathbb{R}^{d}\right)$ and $\left(u_{k}\right)_{k \in \mathbb{N}}$ in $C_{c}^{\infty}(Z)$ converging to $\mathbf{g}, u$ respectively as described in Lemma 5.24. According to Proposition 5.11, each related initial value problem

$$
\partial_{t} f_{k}+\operatorname{div}_{\mathbf{x}}\left(f_{k} \mathbf{v}\right)+\operatorname{div}_{\mathbf{y}}\left(f_{k} \mathbf{g}_{k}\right)=u_{k} f_{k}+w \text { in }[0, T], \quad f_{k}(0)=f_{0}
$$

has a weak solution $f_{k}:[0, T] \longrightarrow L^{2}(Z)$. Estimates (3.), (5.), (8.) in Proposition 5.21 imply

$$
\begin{aligned}
\left\|f_{k}(t)\right\|_{L^{2}(Z)} & \leq e^{C T} \cdot\left(\left\|f_{0}\right\|_{L^{2}(Z)}+\|w\|_{L^{2}(Z)}\right) & & \text { for all } k \in \mathbb{N}, \quad t \in[0, T], \\
e^{f}\left(f_{k}(s), f_{k}(t)\right) & \leq C \cdot|t-s| & & \text { for all } k \in \mathbb{N}, s, t \in[0, T], \\
\sup _{t \in[0, T]} d^{f}\left(f_{k}(t), f_{l}(t)\right) & \longrightarrow 0 & & \text { for } k, l \longrightarrow \infty
\end{aligned}
$$

with a constant $C=\operatorname{const}\left(\left\|f_{0}\right\|_{L^{2}},\|g\|_{L^{2}},\left\|\operatorname{div}_{\mathbf{y}} \mathbf{g}\right\|_{L^{\infty}},\|u\|_{L^{\infty}},\|w\|_{L^{2}}\right)$. Furthermore the subset $\left\{\left|f_{k}(t)\right|^{2} \mid k \in \mathbb{N}, t \in[0, T]\right\} \subseteq L^{1}(Z)$ is tight due to Lemma 5.25. According to Lemma 5.6, there exists a bounded limit function $f:[0, T] \longrightarrow L^{2}(Z)$ with

$$
d^{f}\left(f_{l}(t), f(t)\right) \longrightarrow 0 \text { for } l \longrightarrow \infty \text { and each } t \in[0, T]
$$

By means of the triangle inequality, this convergence proves to be even uniform w.r.t. $t \in[0, T]$ :

$$
\sup _{t \in[0, T]} d^{f}\left(f_{k}(t), f(t)\right) \longrightarrow 0 \quad \text { for } k \longrightarrow \infty
$$

In particular, $f$ is Lipschitz continuous with respect to $\breve{e}^{f}$ and so it is weakly continuous due to Lemma 5.3.

Finally $f:[0, T] \longrightarrow L^{2}(Z)$ proves to satisfy the characteristic condition (32) on weak solutions, i.e. for any $0 \leq t_{1}<t_{2} \leq T, \varphi \in C_{c}^{\infty}(Z)$

$$
\begin{aligned}
\int_{Z} \varphi\left(f\left(t_{2}\right)-f\left(t_{1}\right)\right) d \mathbf{z}= & \int_{t_{1}}^{t_{2}} \int_{Z}\left(f(s, \cdot)\left(\mathbf{v} \cdot \nabla_{\mathbf{x}} \varphi+\mathbf{g} \cdot \nabla_{\mathbf{y}} \varphi+u \varphi\right)\right) d \mathbf{z} d s \\
& +\left(t_{2}-t_{1}\right) \int_{Z} \varphi w d \mathbf{z}
\end{aligned}
$$


Indeed, the general relation $\breve{e}^{f} \leq d^{f}$ and Lemma 5.3 imply $f_{k}(t) \longrightarrow f(t)$ weakly in $L^{2}(Z)$ for each $t \in[0, T]$. For arbitrary $\varepsilon>0$ choose the index $J \in \mathbb{N}$ sufficiently large such that $\left\|g_{l}-g\right\|_{L^{2}(Z)}+\left\|u_{l}-u\right\|_{L^{2}(Z)} \leq \varepsilon$ holds for all $l \geq J$. Fixing any indices $k, l \geq J$, the uniform convergence of $\left(f_{k}\right)_{k \in \mathbb{N}}$ w.r.t. $d^{f}$ leads to

$$
\begin{aligned}
& \left|\int_{t_{1}}^{t_{2}} \int_{Z} f_{k}(s, \cdot) \mathbf{g}_{k} \cdot \nabla_{\mathbf{y}} \varphi d \mathbf{z}-\int_{t_{1}}^{t_{2}} \int_{Z} f(s, \cdot) \mathbf{g} \cdot \nabla_{\mathbf{y}} \varphi d \mathbf{z}\right| \\
& \leq \quad\left|\int_{t_{1}}^{t_{2}} \int_{Z} f_{k}(s, \cdot)\left(\mathbf{g}_{k}-\mathbf{g}_{l}\right) \cdot \nabla_{\mathbf{y}} \varphi d \mathbf{z}\right|+\left|\int_{t_{1}}^{t_{2}} \int_{Z}\left(f_{k}(s, \cdot)-f(s, \cdot)\right) \mathbf{g}_{l} \cdot \nabla_{\mathbf{y}} \varphi d \mathbf{z}\right|+ \\
& \quad\left|\int_{t_{1}}^{t_{2}} \int_{Z} f(s, \cdot)\left(\mathbf{g}_{l}-\mathbf{g}\right) \cdot \nabla_{\mathbf{y}} \varphi d \mathbf{z}\right| \\
& \leq \quad \operatorname{const}\left(\left\|f_{0}\right\|_{L^{2}},\|w\|_{L^{2}},\left\|\nabla_{\mathbf{y}} \varphi\right\|_{L^{\infty}}\right) \quad \cdot\left\|\mathbf{g}_{k}-\mathbf{g}_{l}\right\|_{L^{2}} \\
& \quad+\operatorname{const}\left(\left\|\mathbf{g}_{l} \cdot \nabla_{\mathbf{y}} \varphi\right\|_{W^{1, \infty}}+\left\|\mathbf{g}_{l} \cdot \nabla_{\mathbf{y}} \varphi\right\|_{L^{2}}\right) \cdot \sup _{s \in[0, T]} d^{f}\left(f_{k}(s), f(s)\right) \\
& \quad+\operatorname{const}\left(\left\|f_{0}\right\|_{L^{2}},\|w\|_{L^{2}},\left\|\nabla_{\mathbf{y}} \varphi\right\|_{L^{\infty}}\right) \quad \cdot\left\|\mathbf{g}_{l}-\mathbf{g}\right\|_{L^{2}}
\end{aligned}
$$

and so we obtain

$\lim _{k \rightarrow \infty}\left|\int_{t_{1}}^{t_{2}} \int_{Z}\left(f_{k}(s, \cdot) \mathbf{g}_{k} \cdot \nabla_{\mathbf{y}} \varphi-f(s, \cdot) \mathbf{g} \cdot \nabla_{\mathbf{y}} \varphi\right) d \mathbf{z}\right| \leq \operatorname{const}\left(\left\|f_{0}\right\|_{L^{2}},\|w\|_{L^{2}},\left\|\nabla_{\mathbf{y}} \varphi\right\|_{L^{\infty}}\right) \cdot \varepsilon$

Essentially the same arguments guarantee

$$
\lim _{k \rightarrow \infty}\left|\int_{t_{1}}^{t_{2}} \int_{Z}\left(f_{k}(s, \cdot) u_{k} \varphi-f(s, \cdot) u \varphi\right) d \mathbf{z}\right| \leq \operatorname{const}\left(\left\|f_{0}\right\|_{L^{2}},\|w\|_{L^{2}},\|\varphi\|_{L^{\infty}}\right) \cdot \varepsilon
$$

with $\varepsilon>0$ having being fixed arbitrarily small.

Finally the weak solution $f:[0, T] \longrightarrow L^{2}(Z)$ is unique as a consequence of Proposition 5.7. The uniform convergence w.r.t. $d^{f}$ implies that all estimates in Proposition 5.21 hold for $\vartheta_{\mathbf{g}, u, w}^{f}\left(\cdot, f_{0}\right) \stackrel{\text { Def. }}{=} f(\cdot):[0,1] \longrightarrow L^{2}(Z)($ with $T=1)$. 


\subsection{Nonautonomous linear problem with "less regular" coefficients: Existence of solutions and estimates}

\section{Proposition 5.26}

For any $T \in] 0, \infty\left[\right.$, suppose $f_{0} \in L^{2}(Z), \widehat{w} \in L^{2}(Z)$,

$$
\begin{array}{llll}
\widetilde{\mathrm{g}} & \in L^{1}\left(0, T ; L^{2}\left(Z, \mathbb{R}^{d}\right)\right) \cap L^{\infty}\left(0, T ; L^{\infty}\left(Z, \mathbb{R}^{d}\right)\right), & \partial_{\mathbf{y}} \widetilde{\mathrm{g}} \in L^{\infty}\left(0, T ; L^{\infty}\left(Z, \mathbb{R}^{d \times d}\right)\right), \\
\widetilde{u} \in L^{1}\left(0, T ; L^{2}(Z)\right) & \cap L^{\infty}\left(0, T ; L^{\infty}(Z)\right), & \partial_{\mathbf{y}} \widetilde{u} \in L^{\infty}\left(0, T ; L^{\infty}\left(Z, \mathbb{R}^{d}\right)\right), \\
\widetilde{w} \in L^{\infty}\left(0, T ; L^{2}(Z)\right) & & &
\end{array}
$$

and compact sets $K_{\mathbf{x}} \subseteq \mathbb{R}^{n}, K_{\mathbf{y}} \subseteq Y$ with $|\widetilde{w}(t)| \leq \widehat{w}$ Lebesgue-a.e. in $Z \backslash\left(K_{\mathbf{x}} \times V \times K_{\mathbf{y}}\right)$ for every $t \in[0, T]$.

Then there exists a weak solution $f:[0, T] \longrightarrow L^{2}(Z)$ to the nonautonomous linear transport equation

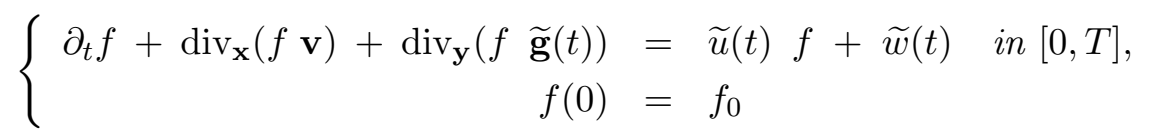

(in the same sense as in Proposition 5.11 about autonomous linear problems), i.e. $f:[0, T] \longrightarrow$ $L^{2}(Z)$ is weakly continuous with $f(0)=f_{0}$ and it holds for any $0 \leq t_{1}<t_{2} \leq T, \varphi \in C_{c}^{1}(Z)$

$$
\begin{aligned}
\int_{Z} \varphi\left(f\left(t_{2}\right)-f\left(t_{1}\right)\right) d \mathbf{z}= & \int_{t_{1}}^{t_{2}} \int_{Z}\left(f(s, \cdot)\left(\mathbf{v} \cdot \nabla_{\mathbf{x}} \varphi+\widetilde{\mathbf{g}}(s) \cdot \nabla_{\mathbf{y}} \varphi+\widetilde{u}(s) \varphi\right)\right) d \mathbf{z} d s \\
& +\int_{t_{1}}^{t_{2}} \int_{Z} \varphi \widetilde{w}(s) d \mathbf{z} d s .
\end{aligned}
$$

In particular, $f$ is continuous w.r.t. $d^{f}$ and, the following estimate is fulfilled for every $t \in[0, T]$

$$
\|f(t)\|_{L^{2}(Z)} \leq\left(\left\|f_{0}\right\|_{L^{2}(Z)}+\int_{0}^{t}\|\widetilde{w}(s)\|_{L^{2}(Z)} d s\right) e^{\operatorname{const}(n) \cdot\left(1+\left\|\operatorname{div}_{\mathbf{y}} \widetilde{\mathbf{g}}\right\|_{L^{\infty}\left(L^{\infty}\right)}+\|\widetilde{u}\|_{L^{\infty}\left(L^{\infty}\right)}\right) \cdot t} .
$$

Remark 5.27 The weak solution of (46) is unique according to Proposition 5.7.

\section{Corollary 5.28}

Let $\widetilde{\mathbf{g}}^{(1)}, \widetilde{\mathbf{g}}^{(2)}, \widetilde{u}^{(1)}, \widetilde{u}^{(2)}$ and $\widetilde{w}^{(1)}, \widetilde{w}^{(2)}$ satisfy the assumptions of Proposition 5.26 respectively. Then the weak solutions $f^{(1)}, f^{(2)}:[0, T] \longrightarrow L^{2}(Z)$ to the respective modification of (46) satisfy for every $t \in[0, T]$

$$
\begin{aligned}
& d^{f}\left(f^{(1)}(t), f^{(2)}(t)\right) \\
& \leq \quad\left(d^{f}\left(f^{(1)}(0), f^{(2)}(0)\right)+\right. \\
& \left.\quad C \cdot \int_{0}^{t}\left\|\left(\widetilde{\mathbf{g}}^{(1)}, \widetilde{u}^{(1)}, \widetilde{w}^{(1)}\right)(s)-\left(\widetilde{\mathbf{g}}^{(2)}, \widetilde{u}^{(2)}, \widetilde{w}^{(2)}\right)(s)\right\|_{L^{2}\left(Z, \mathbb{R}^{d+2}\right)} d s\right) \cdot e^{C t}
\end{aligned}
$$

with a constant $C$ depending only on $\left\|f^{(1)}\right\|_{L^{\infty}\left(0, T ; L^{2}(Z)\right)}$ and

$$
\sup _{i=1,2}\left(\left\|\partial_{\mathbf{y}} \widetilde{\mathbf{g}}^{(i)}\right\|_{L^{\infty}\left(L^{\infty}\right)}+\left\|\widetilde{u}^{(i)}\right\|_{L^{\infty}\left(L^{\infty}\right)}+\left\|\partial_{\mathbf{y}} \widetilde{u}^{(i)}\right\|_{L^{\infty}\left(L^{\infty}\right)}+\left\|\widetilde{w}^{(i)}\right\|_{L^{\infty}\left(L^{2}\right)}\right) .
$$


Proof of Proposition 5.26. Similarly to Lemma 5.24, the standard tool of mollifying with some Dirac sequence leads to sequences

$$
\begin{array}{lll}
\left(\widetilde{\mathbf{g}}_{k}\right)_{k \in \mathbb{N}} & \text { in } & L^{1}\left(0, T ; C_{c}^{1}\left(Z, \mathbb{R}^{d}\right)\right), \\
\left(\widetilde{u}_{k}\right)_{k \in \mathbb{N}} & \text { in } \quad L^{1}\left(0, T ; C^{1}(Z) \cap W^{1, \infty}(Z) \cap L^{2}(Z),\|\cdot\|_{L^{2}(Z)}\right), \\
\left(\widetilde{w}_{k}\right)_{k \in \mathbb{N}} & \text { in } \quad L^{1}\left(0, T ; L^{2}(Z)\right)
\end{array}
$$

whose functions are piecewise constant in time and satisfy

$$
\begin{array}{clll}
\lim _{k \rightarrow \infty} \int_{[0, T]}\left\|\widetilde{\mathbf{g}}-\widetilde{\mathbf{g}}_{k}\right\|_{L^{2}\left(Z, \mathbb{R}^{d}\right)} d t=0, & \sup _{k \in \mathbb{N}} \max _{t \in[0, T]}\left\|\partial_{\mathbf{y}} \widetilde{\mathbf{g}}_{k}(t)\right\|_{L^{\infty}} \leq\left\|\partial_{\mathbf{y}} \widetilde{\mathbf{g}}\right\|_{L^{\infty}\left(L^{\infty}\right)}+\|\widetilde{\mathbf{g}}\|_{L^{\infty}\left(L^{\infty}\right)}, \\
\lim _{k \rightarrow \infty} \int_{[0, T]}\left\|\widetilde{u}-\widetilde{u}_{k}\right\|_{L^{2}(Z)} \quad d t=0, & \sup _{k \in \mathbb{N}} \max _{t \in[0, T]}\left\|\partial_{\mathbf{y}} \widetilde{u}_{k}(t)\right\|_{L^{\infty}} \leq\left\|\partial_{\mathbf{y}} \widetilde{u}\right\|_{L^{\infty}\left(L^{\infty}\right)}, \\
\lim _{k \rightarrow \infty} \int_{[0, T]}\left\|\widetilde{w}-\widetilde{w}_{k}\right\|_{L^{2}(Z)} \quad d t=0, & \sup _{k \in \mathbb{N}} \max _{t \in[0, T]}\left\|\widetilde{w}_{k}(t)\right\|_{L^{2}} \leq\|\widetilde{w}\|_{L^{\infty}\left(L^{2}\right)}, \\
& \forall k, t: \quad\left|\widetilde{w}_{k}(t)\right| \leq|\widehat{w}| \text { a.e. in } \widehat{Z}
\end{array}
$$

with $\widehat{Z}:=Z \backslash\left(K_{\mathbf{x}} \times V \times K_{\mathbf{y}}\right) \subseteq \mathbb{R}^{n} \times V \times Y$.

For each index $k \in \mathbb{N}$, we consider the finite partition $0=t_{k}^{0}<t_{k}^{1}<\ldots<t_{k}^{J_{k}}=T$ such that $\widetilde{\mathbf{g}}_{k}, \widetilde{u}_{k}, \widetilde{w}_{k}$ are constant in every subinterval $\left[t_{k}^{j}, t_{k}^{j+1}[\right.$. Then the approximative solution $f_{k}:[0, T] \longrightarrow L^{2}(Z)$ is constructed by means of Euler method:

$$
\begin{aligned}
f_{k}(0) & :=f_{0}, \\
f_{k}(t) & \left.\left.:=\vartheta_{\widetilde{\mathbf{g}}\left(t_{k}^{j}\right), \widetilde{u}\left(t_{k}^{j}\right), \widetilde{w}\left(t_{k}^{j}\right)}^{f}\left(t-t_{k}^{j}, f\left(t_{k}^{j}\right)\right) \quad \text { for } t \in\right] t_{k}^{j}, t_{k}^{j+1}\right], j=0 \ldots J_{k}-1 .
\end{aligned}
$$

It is the weak solution of the nonautonomous linear transport equation

$$
\partial_{t} f+\operatorname{div}_{\mathbf{x}}(f \mathbf{v})+\operatorname{div}_{\mathbf{y}}\left(f \widetilde{\mathbf{g}}_{k}(t)\right)=\widetilde{u}_{k}(t) f+\widetilde{w}_{k}(t) \quad \text { in }[0, T] .
$$

$f_{k}(\cdot)$ satisfies the a priori estimate (48) as a piecewise consequence of Proposition 5.21 (3.). Moreover $f_{k}(\cdot)$ is even continuous w.r.t. the metric $e^{f}$ according to Proposition 5.21 (6.).

The set of all square values $\left\{\left|f_{l}(t)\right|^{2} \mid t \in[0, T], l \in \mathbb{N}\right\}$ is tight - for the same reasons as in Lemma 5.25. This has two useful consequences: Firstly the ( $L^{2}$ norm) closed convex hull of $\left\{f_{l}(t) \mid t \in[0, T], l \in \mathbb{N}\right\} \subseteq L^{2}(Z)$ is complete w.r.t. the metric $d^{f}$ according to Lemma 5.6. Secondly Proposition 5.5 guarantees the continuity of each function $f_{k}(\cdot), k \in \mathbb{N}$, w.r.t. $d^{f}$. Furthermore the extension of Proposition 5.21 (8.) (to less regular coefficients) implies

$$
d^{f}\left(f_{k}(t), f_{l}(t)\right) \leq C \cdot\left(1+\left\|f_{0}\right\|_{L^{2}(Z)}\right) \cdot \int_{0}^{T}\left(\left\|\widetilde{\mathbf{g}}_{k}-\widetilde{\mathbf{g}}_{l}\right\|_{L^{2}}+\left\|\widetilde{u}_{k}-\widetilde{u}_{l}\right\|_{L^{2}}+\left\|\widetilde{w}_{k}-\widetilde{w}_{l}\right\|_{L^{2}}\right) d s
$$

for every $t \in[0, T]$ with a constant

$$
C=C\left(n,\left\|\partial_{\mathbf{y}} \widetilde{\mathbf{g}}\right\|_{L^{\infty}\left(L^{\infty}\right)},\|\widetilde{u}\|_{L^{\infty}\left(L^{\infty}\right)},\left\|\partial_{\mathbf{y}} \widetilde{u}\right\|_{L^{\infty}\left(L^{\infty}\right)},\|\widetilde{w}\|_{L^{\infty}\left(L^{2}\right)}\right) .
$$

and so $\left(f_{k}\right)_{k \in \mathbb{N}}$ is a uniform Cauchy sequence of continuous functions $[0, T] \longrightarrow\left(L^{2}(Z), d^{f}\right)$. 
Thus there exists a continuous function $f:[0, T] \longrightarrow\left(L^{2}(Z), d^{f}\right)$ with

$$
\sup _{t \in[0, T]} d^{f}\left(f_{k}(t), f(t)\right) \longrightarrow 0 \quad(k \longrightarrow \infty) .
$$

In particular, $f(\cdot)$ is weakly continuous in $L^{2}(Z)$ due to $\breve{e}^{f} \leq d^{f}$ and Lemma 5.3. We conclude from the same arguments that for every $t \in[0, T]$, the sequence $\left(f_{k}(t)\right)_{k \in \mathbb{N}}$ converges to $f(t)$ weakly in $L^{2}(Z)$ and so, $f(t)$ satisfies inequality (48) as well.

It remains to verify that $f(\cdot)$ is a weak solution to the nonautonomous linear transport equation (46). We can use essentially the same limit arguments as in the proof of Proposition 5.23 and just have to take into consideration that the coefficients $\widetilde{\mathbf{g}}(\cdot), \widetilde{u}(\cdot), \widetilde{w}(\cdot)$ depend on time additionally.

Proof of Corollary 5.28. As explained in the proof of Proposition 5.26, the solutions $f^{(1)}, f^{(2)}:[0, T] \longrightarrow L^{2}(Z)$ to the respective modifications of (46) can be approximated by sequences $\left(f_{k}^{(1)}\right)_{k \in \mathbb{N}},\left(f_{k}^{(2)}\right)_{k \in \mathbb{N}}$ of solutions to nonautonomous linear transport equations whose approximative coefficients are piecewise constant in time additionally.

First we consider the case $f^{(1)}(0)=f^{(2)}(0)$.

The extension of Proposition 5.21 (8.) (to less regular coefficients) can again be applied piecewise in time and so we obtain for every $t \in[0, T], k \in \mathbb{N}$

$d^{f}\left(f_{k}^{(1)}(t), f_{k}^{(2)}(t)\right) \leq C \cdot\left(1+\left\|f_{0}^{(1)}\right\|_{L^{2}}\right) \cdot \int_{0}^{T}\left(\left\|\widetilde{\mathbf{g}}_{k}^{(1)}-\widetilde{\mathbf{g}}_{k}^{(2)}\right\|_{L^{2}}+\left\|\widetilde{u}_{k}^{(1)}-\widetilde{u}_{k}^{(2)}\right\|_{L^{2}}+\left\|\widetilde{w}_{k}^{(1)}-\widetilde{w}_{k}^{(2)}\right\|_{L^{2}}\right) d s$ with a constant $C=C\left(n,\left\|\partial_{\mathbf{y}} \widetilde{\mathbf{g}}^{(j)}\right\|_{L^{\infty}\left(L^{\infty}\right)},\left\|\widetilde{u}^{(j)}\right\|_{L^{\infty}\left(L^{\infty}\right)},\left\|\partial_{\mathbf{y}} \widetilde{u}^{(j)}\right\|_{L^{\infty}\left(L^{\infty}\right)},\left\|\widetilde{w}^{(j)}\right\|_{L^{\infty}\left(L^{2}\right)}\right)<\infty$. The limit for $k \longrightarrow \infty$ leads to the claimed upper estimate of $d^{f}\left(f^{(1)}(t), f^{(2)}(t)\right)$ for each $t \in[0, T]$.

The more general case $f^{(1)}(0) \neq f^{(2)}(0)$ results from the preceding special case applied to $f^{(1)}(\cdot)$ and the solution $\widehat{f}:[0, T] \longrightarrow L^{2}(Z)$ to the linear auxiliary problem

$$
\left\{\begin{aligned}
\partial_{t} \widehat{f}+\operatorname{div}_{\mathbf{x}}(\widehat{f} \mathbf{v})+\operatorname{div}_{\mathbf{y}}\left(\widehat{f} \widetilde{\mathbf{g}}^{(2)}(t)\right) & =\widetilde{u}^{(2)}(t) \widehat{f}+\widetilde{w}^{(2)}(t) \quad \text { in }[0, T], \\
\widehat{f}(0) & =f_{0}^{(1)}
\end{aligned}\right.
$$

Indeed,

$d^{f}\left(f^{(1)}(t), \widehat{f}(t)\right) \leq C \cdot\left(1+\left\|f_{0}^{(1)}\right\|_{L^{2}}\right) \cdot \int_{0}^{T}\left(\left\|\widetilde{\mathbf{g}}^{(1)}-\widetilde{\mathbf{g}}^{(2)}\right\|_{L^{2}}+\left\|\widetilde{u}^{(1)}-\widetilde{u}^{(2)}\right\|_{L^{2}}+\left\|\widetilde{w}^{(1)}-\widetilde{w}^{(2)}\right\|_{L^{2}}\right) d s$ holds for every $t \in[0, T]$. Moreover, we can conclude from extended Proposition 5.21 (7.) in the same piecewise way

$$
d^{f}\left(\widehat{f}(t), f^{(2)}(t)\right) \leq d^{f}\left(\widehat{f}(0), f^{(2)}(0)\right) \cdot e^{c t}=d^{f}\left(f^{(1)}(0), f^{(2)}(0)\right) \cdot e^{c t}
$$

for every $t \in[0, T]$ with a constant $c$ depending only on the bounds of coefficients (and their divergence w.r.t. $\mathbf{y})$. The final estimate results from the triangle inequality of metric $d^{f}$. 


\section{The subproblems for $Q$ and $L$}

Similarly to $\S 5.7$, we focus on the nonautonomous linear problems for $Q$ and $L$ separately and collect the corresponding standard results briefly.

\subsection{The nonautonomous linear problem for $Q$ : Unique solutions via Bochner integrals}

\section{Proposition 6.1}

For every $q(\cdot) \in L^{1}\left(0, T ; L^{2}\left(\mathbb{R}^{n} \times \mathbb{S}^{n-1}\right)\right)$ and initial function $Q_{0} \in L^{2}\left(\mathbb{R}^{n} \times \mathbb{S}^{n-1}\right)$, there exists a weak solution $Q:[0, T] \longrightarrow L^{2}\left(\mathbb{R}^{n} \times \mathbb{S}^{n-1}\right)$ of

$$
\left\{\begin{aligned}
\partial_{t} Q(t) & =q(t) \quad \text { in }[0, T], \\
Q(0) & =Q_{0}
\end{aligned}\right.
$$

in the sense that $Q(\cdot)$ is weakly continuous and satisfies for any $\phi \in C_{c}^{1}\left(\left[0, T\left[\times \mathbb{R}^{n} \times \mathbb{S}^{n-1}\right)\right.\right.$

$$
\int_{\mathbb{R}^{n} \times \mathbb{S}^{n-1}}\left(\phi(T, \zeta) Q(T, \zeta)-\phi(0, \zeta) Q_{0}(\zeta)\right) d \zeta=\int_{0}^{T} \int_{\mathbb{R}^{n} \times \mathbb{S}^{n-1}}\left(\partial_{s} \phi \cdot Q+\phi \cdot q\right) d \zeta d s
$$

It is unique and can be represented in form of a Bochner integral:

$$
Q(t)=Q_{0}+\int_{0}^{t} q(s) d s .
$$

Corollary 6.2 For any functions $q^{(1)}, q^{(2)} \in L^{1}\left(0, T ; L^{2}\left(\mathbb{R}^{n} \times \mathbb{S}^{n-1}\right)\right)$ and $Q_{0}^{(1)}, Q_{0}^{(2)} \in$ $L^{2}\left(\mathbb{R}^{n} \times \mathbb{S}^{n-1}\right)$, let $Q^{(i)}:[0, T] \longrightarrow L^{2}\left(\mathbb{R}^{n} \times \mathbb{S}^{n-1}\right)$ denote the unique solution of

$$
\left\{\begin{aligned}
\partial_{t} Q^{(i)}(t) & =q^{(i)}(t) \quad \text { in }[0, T], \\
Q^{(i)}(0) & =Q_{0}^{(i)}
\end{aligned}\right.
$$

for $i=1,2$ respectively. Then the following estimate holds for every $t \in[0, T]$

$$
\left\|Q^{(1)}(t)-Q^{(2)}(t)\right\|_{L^{2}\left(\mathbb{R}^{n} \times \mathbb{S}^{n-1}\right)} \leq\left\|Q_{0}^{(1)}-Q_{0}^{(2)}\right\|_{L^{2}\left(\mathbb{R}^{n} \times \mathbb{S}^{n-1}\right)}+\int_{0}^{t}\left\|q^{(1)}(s)-q^{(2)}(s)\right\|_{L^{2}} d s .
$$

Proof of Proposition 6.1. For every Bochner integrable $q:[0, T] \longrightarrow L^{2}\left(\mathbb{R}^{n} \times \mathbb{S}^{n-1}\right)$,

$$
[0, T] \longrightarrow L^{2}\left(\mathbb{R}^{n} \times \mathbb{S}^{n-1}\right), \quad t \longmapsto \int_{0}^{t} q(s) d s
$$

is differentiable Lebesgue-almost everywhere in $[0, T]$ as a consequence of [31, Theorem 3.8.5]. Furthermore it is bounded by the Lebesgue integral of the norm $s \longmapsto\|q(s)\|_{L^{2}\left(\mathbb{R}^{n} \times \mathbb{S}^{n-1}\right)}$ due to [31, Theorem 3.7.6] and so, it is norm-continuous. Thus, $[0, T] \longrightarrow L^{2}\left(\mathbb{R}^{n} \times \mathbb{S}^{n-1}\right), t \longmapsto$ $Q_{0}+\int_{0}^{t} q(s) d s$ is a weak solution. 
It remains to show the uniqueness of weak solutions. The difference $\Delta:[0, T] \longrightarrow L^{2}\left(\mathbb{R}^{n} \times \mathbb{S}^{n-1}\right)$ of any two weak solutions of initial value problem (49) is weakly continuous and satisfies for every $\phi \in C_{c}^{1}\left(\left[0, T\left[\times \mathbb{R}^{n} \times \mathbb{S}^{n-1}\right)\right.\right.$

$$
\int_{\mathbb{R}^{n} \times \mathbb{S}^{n-1}} \phi(T, \zeta) \cdot \Delta(T, \zeta) d \zeta=\int_{0}^{T} \int_{\mathbb{R}^{n} \times \mathbb{S}^{n-1}} \partial_{s} \phi(s, \zeta) \cdot \Delta(s, \zeta) d \zeta d s .
$$

For any function $\varphi_{0} \in L^{2}\left(\mathbb{R}^{n} \times \mathbb{S}^{n-1}\right)$, we conclude that the Lebesgue integrable function

$$
\left[0, T\left[\longrightarrow \mathbb{R}, \quad t \longmapsto \int_{\mathbb{R}^{n} \times \mathbb{S}^{n-1}} \varphi_{0}(\zeta) \cdot \Delta(t, \zeta) d \zeta\right.\right.
$$

has the distributional distribution identical 0 and so, it is constant Lebesgue-almost everywhere. The weak continuity of $\Delta(\cdot)$ and the initial condition $\Delta(0)=0$ imply $\Delta(\cdot)=0$ in $[0, T]$, i.e. the weak solution of (49) is unique.

\subsection{The nonautonomous semilinear reaction-diffusion problem for $L$}

\section{Proposition 6.3 (Solutions to semilinear parabolic problems in $\mathbb{R}^{n}$ )}

Suppose for $R:[0, T] \times L^{2}\left(\mathbb{R}^{n}\right) \longrightarrow L^{2}\left(\mathbb{R}^{n}\right)$

1. for every $u \in L^{2}(\Omega)$, the function $R(\cdot, u):[0, T] \longrightarrow L^{2}\left(\mathbb{R}^{n}\right)$ is measurable,

2. there exists some $\lambda \in L^{1}([0, T])$ such that for every $t \in[0, T]$, the function $R(t, \cdot)$ : $L^{2}\left(\mathbb{R}^{n}\right) \longrightarrow L^{2}\left(\mathbb{R}^{n}\right)$ is $\lambda(t)$-Lipschitz continuous.

Then for every initial $L_{0} \in L^{2}\left(\mathbb{R}^{n}\right)$, there exists a unique mild solution $L \in C^{0}\left([0, T], L^{2}\left(\mathbb{R}^{n}\right)\right)$ to nonautonomous semilinear evolution problem

$$
\left\{\begin{aligned}
\partial_{t} L & =\Delta_{\mathbf{x}} L+R(t, L) & & \text { in }] 0, T\left[\times \mathbb{R}^{n}\right. \\
L(0) & =L_{0} & & \text { in } \mathbb{R}^{n}
\end{aligned}\right.
$$

Furthermore $L$ is also weak solution.

Remark 6.4 This proposition is a special case of [59, Theorem 3.2] or, equivalently in our context, [27, Theorem 1.2].

In more details, Tolstogonov [59] investigates evolution inclusions even with (possibly nonlinear) semigroups which are generated by an $m$-dissipative operator. His terms of lower order are specified as a multivalued map of time and state whereas we have the just single-valued function $R$ here. [59, Theorem 3.1] guarantees the existence of a so-called integral solution for a much broader class of generating operators.

[59, Theorem 3.2] and [27, Theorem 1.2] consider an infinitesimal generator of a strongly continuous semigroup of bounded linear operators and provide mild solutions. We apply this result to the Laplace operator and conclude uniqueness from assumption (2) by means of Gronwall's inequality. 


\section{Proposition 6.5 (The explicit weak solution in the whole space $\mathbb{R}^{n}$ )}

For any $\beta \geq 0$ set $\Gamma:] 0, \infty\left[\times \mathbb{R}^{n} \longrightarrow \mathbb{R}, \quad(t, \mathbf{x}) \longmapsto \frac{1}{(4 \pi t)^{\frac{n}{2}}} \cdot \exp \left(-\frac{|\mathbf{x}|^{2}}{4 t}-\beta t\right)\right.$. Choose $\kappa, T>0, L_{0} \in L^{2}\left(\mathbb{R}^{n}\right)$ and $\sigma \in L^{1}\left(0, T ; L^{2}\left(\mathbb{R}^{n}\right)\right)$ arbitrarily.

Then the function $L:[0, T] \times \mathbb{R}^{n} \longrightarrow \mathbb{R}$,

$$
L(t, \mathbf{x}):=\int_{\mathbb{R}^{n}} \Gamma(\kappa t, \mathbf{x}-\zeta) \cdot L_{0}(\zeta) d \zeta+\int_{0}^{t} \int_{\mathbb{R}^{n}} \Gamma(\kappa(t-s), \mathbf{x}-\zeta) \cdot \sigma(s, \zeta) d \zeta d s
$$

is the unique weak solution of the nonautonomous linear reaction-diffusion equation

$$
\left\{\begin{aligned}
\partial_{t} L & =\kappa \cdot \Delta_{\mathbf{x}} L-\beta L+\sigma & & \text { in }] 0, T\left[\times \mathbb{R}^{n}\right. \\
L(0) & =L_{0} & & \text { in } \mathbb{R}^{n}
\end{aligned}\right.
$$

In particular, $L(t, \cdot) \in W^{1,2}\left(\mathbb{R}^{n}\right)$ holds for every $\left.\left.t \in\right] 0, T\right]$ if $\sigma \in L^{2}\left(0, T ; L^{2}\left(\mathbb{R}^{n}\right)\right)$ in addition.

Corollary 6.6 (A priori estimates) $\quad$ For $\beta \geq 0, \kappa, T>0$ and $\sigma_{1}, \sigma_{2} \in L^{1}\left(0, T ; L^{2}\left(\mathbb{R}^{n}\right)\right)$, let $L_{1}, L_{2}:[0, T] \longrightarrow L^{2}\left(\mathbb{R}^{n}\right)$ denote the unique weak solutions of $\partial_{t} L_{j}=\kappa \cdot \Delta_{\mathbf{x}} L_{j}-\beta L_{j}+\sigma_{j}$ $(j=1,2)$. Then the following estimates are satisfied for every $t \in] 0, T]$

$$
\begin{aligned}
& \left\|L_{1}(t)-L_{2}(t)\right\|_{L^{2}\left(\mathbb{R}^{n}\right)} \leq\left\|L_{1}(0)-L_{2}(0)\right\|_{L^{2}}+\operatorname{const}(\beta, \kappa, n) \cdot\left\|\sigma_{1}-\sigma_{2}\right\|_{L^{1}\left(0, T ; L^{2}\right)} \\
& \left\|\nabla_{\mathbf{x}}\left(L_{1}-L_{2}\right)\right\|_{L^{1}\left(0, T ; L^{2}\right)} \leq \operatorname{const}(\beta, \kappa, n) \cdot \sqrt{t} \cdot\left(\left\|L_{1}(0)-L_{2}(0)\right\|_{L^{2}}+\left\|\sigma_{1}-\sigma_{2}\right\|_{L^{1}\left(L^{2}\right)}\right)
\end{aligned}
$$

If $\sigma_{1}-\sigma_{2} \in L^{\infty}\left(0, T ; L^{2}\left(\mathbb{R}^{n}\right)\right)$ in addition, then the spatial gradients fulfil for every $\left.\left.t \in\right] 0, T\right]$

$\left\|\nabla_{\mathbf{x}}\left(L_{1}(t)-L_{2}(t)\right)\right\|_{L^{2}\left(\mathbb{R}^{n}\right)} \leq \operatorname{const}(\beta, \kappa, n) \cdot\left(t^{-\frac{1}{2}}\left\|L_{1}(0)-L_{2}(0)\right\|_{L^{2}}+\sqrt{t}\left\|\sigma_{1}-\sigma_{2}\right\|_{L^{\infty}\left(0, T ; L^{2}\right)}\right)$

Proof of Proposition 6.5. If we assume $L_{0} \in C_{c}^{0}\left(\mathbb{R}^{n}\right)$ and $\sigma \in C_{c}^{2}\left([0, T] \times \mathbb{R}^{n}\right)$ in addition, then it is well known that equation (51) specifies the unique classical solution of parabolic problem (52) (see, e.g., $[22, \S 2.3]$ ). Furthermore, the following estimates hold for every $t \in] 0, T]$

$$
\begin{aligned}
\|\Gamma(\kappa t, \cdot)\|_{L^{1}\left(\mathbb{R}^{n}\right)} & =e^{-\beta \kappa t} \\
\left\|\nabla_{\mathbf{x}} \Gamma(\kappa t, \cdot)\right\|_{L^{1}\left(\mathbb{R}^{n}\right)} & \leq \operatorname{const}(\beta, \kappa, n) \cdot t^{-\frac{1}{2}}
\end{aligned}
$$

In the special case $\sigma \equiv 0$ (i.e., $\partial_{t} L=\kappa \cdot \Delta_{\mathbf{x}} L-\beta L$ ), we conclude from the standard rules of convolution (e.g., $\left[38, \S\right.$ VIII.2]) that the function $L:[0, T] \times \mathbb{R}^{n} \longrightarrow \mathbb{R}$ defined in equation (51) satisfies for every $t \in] 0, T]$

$$
\begin{aligned}
\|L(t, \cdot)\|_{L^{2}\left(\mathbb{R}^{n}\right)} & \leq\|\Gamma(\kappa t, \cdot)\|_{L^{1}\left(\mathbb{R}^{n}\right)} \cdot\left\|L_{0}\right\|_{L^{2}\left(\mathbb{R}^{n}\right)} \leq \quad\left\|L_{0}\right\|_{L^{2}\left(\mathbb{R}^{n}\right)} \\
\left\|\nabla_{\mathbf{x}} L(t, \cdot)\right\|_{L^{2}\left(\mathbb{R}^{n}\right)} & \leq\left\|\nabla_{\mathbf{X}} \Gamma(\kappa t, \cdot)\right\|_{L^{1}\left(\mathbb{R}^{n}\right)} \cdot\left\|L_{0}\right\|_{L^{2}\left(\mathbb{R}^{n}\right)} \leq \operatorname{const}(\beta, \kappa, n) \cdot t^{-\frac{1}{2}} \cdot\left\|L_{0}\right\|_{L^{2}\left(\mathbb{R}^{n}\right)}
\end{aligned}
$$


For the more general case of nonhomogeneous parabolic equations (i.e., here arbitrary $\sigma \in$ $\left.C_{c}^{2}\left([0, T] \times \mathbb{R}^{n}\right)\right)$, the variation of constants formula leads to

$$
\begin{aligned}
\|L(t, \cdot)\|_{L^{2}\left(\mathbb{R}^{n}\right)} & \leq \operatorname{const}(\beta, \kappa, n) \quad \cdot\left(\left\|L_{0}\right\|_{L^{2}\left(\mathbb{R}^{n}\right)}+\quad\|\sigma\|_{L^{1}\left(0, T ; L^{2}\left(\mathbb{R}^{n}\right)\right)}\right) \\
\left\|\nabla_{\mathbf{X}} L(t, \cdot)\right\|_{L^{2}\left(\mathbb{R}^{n}\right)} & \leq \operatorname{const}(\beta, \kappa, n) \cdot\left(t^{-\frac{1}{2}} \cdot\left\|L_{0}\right\|_{L^{2}\left(\mathbb{R}^{n}\right)}+\int_{0}^{t}(t-s)^{-\frac{1}{2}} \cdot\|\sigma(s, \cdot)\|_{L^{2}\left(\mathbb{R}^{n}\right)} d s\right)
\end{aligned}
$$

for every $t \in] 0, T]$ and so, we obtain due to Fubini's theorem

$$
\begin{aligned}
& \left\|\nabla_{\mathbf{x}} L\right\|_{L^{1}\left(0, T ; L^{2}\left(\mathbb{R}^{n}\right)\right)}=\int_{0}^{T}\left\|\nabla_{\mathbf{x}} L(t, \cdot)\right\|_{L^{2}\left(\mathbb{R}^{n}\right)} d t \\
& \leq \operatorname{const}(\beta, \kappa, n) \cdot\left(\sqrt{T} \cdot\left\|L_{0}\right\|_{L^{2}\left(\mathbb{R}^{n}\right)}+\int_{\substack{(s, t) \in[0, T]^{2}: \\
s \leq t}}(t-s)^{-\frac{1}{2}} \cdot\|\sigma(s, \cdot)\|_{L^{2}\left(\mathbb{R}^{n}\right)} d(s, t)\right) \\
& \leq \operatorname{const}(\beta, \kappa, n) \cdot\left(\sqrt{T} \cdot\left\|L_{0}\right\|_{L^{2}\left(\mathbb{R}^{n}\right)}+\int_{0}^{T} \sqrt{T-s} \cdot\|\sigma(s, \cdot)\|_{L^{2}\left(\mathbb{R}^{n}\right)} d s\right) \\
& \leq \operatorname{const}(\beta, \kappa, n) \cdot \sqrt{T} \cdot\left(\left\|L_{0}\right\|_{L^{2}\left(\mathbb{R}^{n}\right)}+\|\sigma\|_{L^{1}\left(0, T ; L^{2}\left(\mathbb{R}^{n}\right)\right)}\right) .
\end{aligned}
$$

For arbitrary $L_{0} \in L^{2}\left(\mathbb{R}^{n}\right)$ and $\sigma \in L^{1}\left(0, T ; L^{2}\left(\mathbb{R}^{n}\right)\right)$, consider two sequences in $C_{c}^{0}\left(\mathbb{R}^{n}\right)$ and $C_{c}^{2}\left([0, T] \times \mathbb{R}^{n}\right)$ respectively approximating the given data $L_{0}, \sigma$. The linearity of the reaction-diffusion equation and the last estimates imply that the related solutions form a Cauchy sequence both in $L^{1}\left(0, T ; W^{1,2}\left(\mathbb{R}^{n}\right)\right)$ and w.r.t. supremum norm in $C^{0}\left([0, T], L^{2}\left(\mathbb{R}^{n}\right)\right)$. Hence the limit provides a function $L:[0, T] \times \mathbb{R}^{n} \longrightarrow \mathbb{R}$ with the following properties:

- $\|L\|_{L^{1}\left(0, T ; W^{1,2}\left(\mathbb{R}^{n}\right)\right)} \leq \operatorname{const}(\beta, \kappa, n, T) \cdot\left(\left\|L_{0}\right\|_{L^{2}\left(\mathbb{R}^{n}\right)}+\|\sigma\|_{L^{1}\left(0, T ; L^{2}\left(\mathbb{R}^{n}\right)\right)}\right)$,

- $[0, T] \longrightarrow L^{2}\left(\mathbb{R}^{n}\right), t \longmapsto L(t, \cdot)$ is continuous with $L(0)=L_{0}$,

- $L$ is a weak solution to the nonautonomous linear parabolic equation (52).

Finally it remains to prove that weak solutions of initial value problem (52) are unique. If $\beta>0$, then the energy estimate method in combination with Gronwall's inequality leads to

$$
\left\|L_{1}(t)-L_{2}(t)\right\|_{L^{2}\left(\mathbb{R}^{n}\right)} \leq\left\|L_{1}(0)-L_{2}(0)\right\|_{L^{2}\left(\mathbb{R}^{n}\right)} \cdot e^{\operatorname{const}(\beta) \cdot t}
$$

for the difference of any two weak solutions $L_{1}, L_{2}$ and every $t \in[0, T]$. In the last case $\beta=0$, each weak solution $L:[0, T] \longrightarrow L^{2}\left(\mathbb{R}^{n}\right)$ of the original problem (52) induces a weak solution $\widetilde{L}:[0, T] \longrightarrow L^{2}\left(\mathbb{R}^{n}\right)$ to the auxiliary problem

$$
\partial_{t} \widetilde{L}=\kappa \cdot \Delta_{\mathbf{x}} \widetilde{L}-\widetilde{L}+\sigma \quad \text { in }[0, T], \quad \widetilde{L}(0)=L_{0}
$$

by means of $\widetilde{L}(t):=e^{-t} \cdot L(t)$ and, the latter is unique. 


\section{Proof of main statements in $\S 3$}

In this section we always suppose the hypotheses of Theorem 3.2.

\subsection{Proof of Theorem 3.2 by means of successive approximation}

Consider the space $\mathcal{X}_{T}$ of function tuples $(f, Q, L)$ with

$$
\begin{aligned}
f & \in C^{0}\left([0, T],\left(L^{2}(Z), d^{f}\right)\right) \cap L^{\infty}\left(0, T ; L^{2}(Z)\right), \\
Q & \in C^{0}\left([0, T], L^{2}\left(\mathbb{R}^{n} \times \mathbb{S}^{n-1}\right)\right), \\
L & \in C^{0}\left([0, T], L^{2}\left(\mathbb{R}^{n}\right)\right) \quad \text { s.t. } \operatorname{ess} \sup _{t \in[0, T]} \sqrt{t} \cdot\|L(t)\|_{W^{1,2}\left(\mathbb{R}^{n}\right)}<\infty .
\end{aligned}
$$

Fix the initial functions $f_{0} \in L^{2}(Z), Q_{0} \in L^{2}\left(\mathbb{R}^{n} \times \mathbb{S}^{n-1}\right)$ and $L_{0} \in L^{2}\left(\mathbb{R}^{n}\right)$ arbitrarily. The method of successive approximation induces a map

$$
\mathcal{L}: \mathcal{X}_{T} \longrightarrow \mathcal{X}_{T}, \quad(\widetilde{f}, \widetilde{Q}, \widetilde{L}) \longmapsto(f, Q, L)
$$

in the following way: For $(\widetilde{f}, \widetilde{Q}, \widetilde{L}) \in \mathcal{X}_{T}$ given, $f:[0, T] \longrightarrow L^{2}(Z), Q:[0, T] \longrightarrow L^{2}\left(\mathbb{R}^{n} \times \mathbb{S}^{n-1}\right)$ and $L:] 0, T] \longrightarrow W^{1,2}\left(\mathbb{R}^{n}\right)$ are the unique weak solutions of nonautonomous (partially semi-) linear initial value problem

$$
\left\{\begin{aligned}
\partial_{t} f+\operatorname{div}_{\mathbf{x}}(f \mathbf{v})+\operatorname{div}_{\mathbf{y}}(f \mathcal{G}(t, \widetilde{f}, \widetilde{Q}, \widetilde{L})) & =\mathcal{U}(t, \widetilde{f}, \widetilde{Q}, \widetilde{L}) f+\mathcal{W}(t, \widetilde{f}, \widetilde{Q}, \widetilde{L}), & & f(0)=f_{0}, \\
\partial_{t} Q & =\mathcal{T}(t, \widetilde{f}, \widetilde{Q}, \widetilde{L}), & Q(0) & =Q_{0}, \\
\partial_{t} L & =\kappa_{L} \Delta_{\mathbf{x}} L+\mathcal{R}(t, \widetilde{f}, \widetilde{Q}, L), & & L(0)=L_{0}
\end{aligned}\right.
$$

by means of Propositions 5.26, 6.1 and 6.5 respectively. Indeed, the time-dependent coefficients $t \longmapsto \mathcal{G}(t, \widetilde{f}(t), \widetilde{Q}(t), \widetilde{L}(t))$ etc. satisfy the assumptions of these three propositions and so, $\mathcal{L}: \mathcal{X}_{T} \longrightarrow \mathcal{X}_{T}$ is well-defined.

In regard to Banach's fixed point theorem, we now want to conclude its Lipschitz continuity from assumption (iv) of Theorem 3.2. This requires some a priori bounds depending on the initial functions $f_{0}, Q_{0}, L_{0}$ :

\section{Lemma 7.1 (A priori bounds)}

1. There are constants $\gamma_{f} \geq\left\|f_{0}\right\|_{L^{2}(Z)}, \rho_{f}>0$ both depending on $T,\left\|f_{0}\right\|_{L^{2}(Z)}$ and the bounds in assumption $(i)$ such that for every $(\widetilde{f}, \widetilde{Q}, \widetilde{L}) \in \mathcal{X}_{T}$ with $\|\widetilde{f}(t)\|_{L^{2}(Z)} \leq \rho_{f} \cdot e^{\gamma_{f} \cdot t}$ for all $t \in[0, T]$, the first component $f(\cdot)$ of $\mathcal{L}(\widetilde{f}, \widetilde{Q}, \widetilde{L}) \in \mathcal{X}_{T}$ satisfies

$$
\|f(t)\|_{L^{2}(Z)} \leq \rho_{f} \cdot e^{\gamma_{f} \cdot t} \quad \text { for every } t \in[0, T] .
$$

2. There exists a radius $\rho_{Q}>0$ which depends on $\left\|Q_{0}\right\|_{L^{2}\left(\mathbb{R}^{n} \times \mathbb{S}^{n-1}\right)}, T, \gamma_{f}, \rho_{f}$ such that for each $(\widetilde{f}, \widetilde{Q}, \widetilde{L}) \in \mathcal{X}_{T}$ with $\|\widetilde{f}\|_{L^{\infty}\left(0, T ; L^{2}(Z)\right)} \leq \rho_{f} \cdot e^{\gamma_{f} T}$ the tuple $(f, Q, L):=\mathcal{L}(\widetilde{f}, \widetilde{Q}, \widetilde{L}) \in$ $\mathcal{X}_{T}$ satisfies

$$
\|Q(t)\|_{L^{2}\left(\mathbb{R}^{n} \times \mathbb{S}^{n-1}\right)} \leq \rho_{Q} \quad \text { for every } t \in[0, T] .
$$


3. There exists a radius $\rho_{L}>0$ depending on $\left\|L_{0}\right\|_{L^{2}\left(\mathbb{R}^{n}\right)}, T, \gamma_{f}, \rho_{f}, \rho_{Q}$ such that for each $(\widetilde{f}, \widetilde{Q}, \widetilde{L}) \in \mathcal{X}_{T}$ with $\|\widetilde{f}\|_{L^{\infty}\left(0, T ; L^{2}(Z)\right)} \leq \rho_{f} \cdot e^{\gamma_{f} T}$ and $\|\widetilde{Q}\|_{L^{\infty}\left(0, T ; L^{2}\left(\mathbb{R}^{n} \times \mathbb{S}^{n-1}\right)\right)} \leq \rho_{Q}$, the tuple $(f, Q, L):=\mathcal{L}(\widetilde{f}, \widetilde{Q}, \widetilde{L}) \in \mathcal{X}_{T}$ satisfies

$$
\left.\left.\|L(t)\|_{W^{1,2}\left(\mathbb{R}^{n}\right)} \leq \rho_{L} \cdot\left(1+t^{-\frac{1}{2}}\right) \quad \text { for every } t \in\right] 0, T\right] .
$$

Proof. Statement (1.) results from a priori estimate (48) and assumption (i). Indeed,

$$
\begin{aligned}
\|f(t)\|_{L^{2}(Z)} & \leq\left(\left\|f_{0}\right\|_{L^{2}(Z)}+\int_{0}^{t}\|\mathcal{W}(s, \widetilde{f}, \widetilde{Q}, \widetilde{L})\|_{L^{2}(Z)} d s\right) \cdot e^{\mathrm{const} \cdot t} \\
& \leq\left(\left\|f_{0}\right\|_{L^{2}(Z)}+\int_{0}^{t} \mathrm{const} \cdot\left(1+\|\widetilde{f}(s)\|_{L^{2}(Z)}\right) d s\right) \cdot e^{\mathrm{const} \cdot t} \\
& \leq\left(\left\|f_{0}\right\|_{L^{2}(Z)}+\mathrm{const} \cdot T\right) \cdot e^{\mathrm{const} \cdot T}+\mathrm{const} \cdot e^{\mathrm{const} \cdot T} \cdot \int_{0}^{t}\|\widetilde{f}(s)\|_{L^{2}(Z)} d s .
\end{aligned}
$$

We are looking for a continuous auxiliary function $\psi:[0, T] \longrightarrow[0, \infty[$ such that

$$
\|\widetilde{f}(s)\|_{L^{2}(Z)} \leq \psi(s) \text { for a.e. } s \in[0, T] \quad \Longrightarrow \quad\|f(t)\|_{L^{2}(Z)} \leq \psi(t) \text { for all } t \in[0, T] .
$$

The preceding estimate for $\|f(t)\|_{L^{2}(Z)}$ provides the sufficient condition

$$
\psi(t)=\left(\left\|f_{0}\right\|_{L^{2}(Z)}+\mathrm{const} \cdot T\right) \cdot e^{\mathrm{const} \cdot T}+\operatorname{const} \cdot e^{\mathrm{const} \cdot T} \cdot \int_{0}^{t} \psi(s) d s
$$

for every $t \in[0, T]$ and, this integral equation has the explicit solution

$$
\psi(t)=\left(\left\|f_{0}\right\|_{L^{2}(Z)}+\mathrm{const} \cdot T\right) \cdot e^{\mathrm{const} \cdot T} \cdot e^{\operatorname{const}(T) \cdot t} .
$$

In regard to statement (2.), assumption (ii) implies

$$
\|Q(t)\|_{L^{2}\left(\mathbb{R}^{n} \times \mathbb{S}^{n-1}\right)} \leq\left\|Q_{0}\right\|_{L^{2}\left(\mathbb{R}^{n} \times \mathbb{S}^{n-1}\right)}+\int_{0}^{t} C_{\gamma_{f}, \rho_{f}} \cdot\left(1+\|Q(s)\|_{L^{2}\left(\mathbb{R}^{n} \times \mathbb{S}^{n-1}\right)}\right) d s
$$

for any $t \in[0, T]$ and, Gronwall's inequality leads to an explicit bound

$$
\|Q(t)\|_{L^{2}\left(\mathbb{R}^{n} \times \mathbb{S}^{n-1}\right)} \leq \operatorname{const}\left(\left\|Q_{0}\right\|_{L^{2}\left(\mathbb{R}^{n} \times \mathbb{S}^{n-1}\right)}, C_{\gamma_{f}, \rho_{f}}, T\right)=: \rho_{Q} \text { for every } t \in[0, T] .
$$

Similarly statement (3.) results from Corollary 6.6 and assumption (ii) by means of Gronwall's inequality, i.e. with constants $c=\operatorname{const}\left(\kappa_{L}, n, T, \gamma_{f}, \rho_{f}, \rho_{Q}\right)$

$$
\begin{aligned}
\|L(t)\|_{L^{2}\left(\mathbb{R}^{n}\right)} & \leq c \cdot\left(\left\|L_{0}\right\|_{L^{2}\left(\mathbb{R}^{n}\right)}+\int_{0}^{t}\left(1+\|L(s)\|_{L^{2}\left(\mathbb{R}^{n}\right)}\right) d s\right) \\
\Longrightarrow\|L(t)\|_{L^{2}\left(\mathbb{R}^{n}\right)} & \leq c \cdot\left(\left\|L_{0}\right\|_{L^{2}\left(\mathbb{R}^{n}\right)}+t\right) \cdot e^{c t} .
\end{aligned}
$$

This implies for every $t \in[0, T]$

$$
\|\mathcal{R}(t, \widetilde{f}(t), \widetilde{Q}(t), L(t))\|_{L^{2}\left(\mathbb{R}^{n}\right)} \leq C_{\max \left\{\rho_{f} \cdot e^{\gamma_{f} T}, \rho_{Q}\right\}} \cdot\left(1+c \cdot\left(\left\|L_{0}\right\|_{L^{2}\left(\mathbb{R}^{n}\right)}+T\right) \cdot e^{c T}\right)=: c_{1} .
$$

Finally we conclude from the last inequality in Corollary 6.6 for every $t \in] 0, T]$

$$
\begin{aligned}
\left\|\nabla_{\mathbf{x}} L(t)\right\|_{L^{2}\left(\mathbb{R}^{n}\right)} & \leq \operatorname{const}\left(\kappa_{L}, n\right) \cdot\left(t^{-\frac{1}{2}}\left\|L_{0}\right\|_{L^{2}\left(\mathbb{R}^{n}\right)}+c_{1} \sqrt{t}\right) \\
\Longrightarrow \quad\|L(t)\|_{W^{1,2}\left(\mathbb{R}^{n}\right)} & \leq \operatorname{const}\left(\kappa_{L}, n, T, \gamma_{f}, \rho_{f}, \rho_{Q},\left\|L_{0}\right\|_{L^{2}\left(\mathbb{R}^{n}\right)}\right) \cdot\left(1+t^{-\frac{1}{2}}\right)
\end{aligned}
$$


Consider the subset

$$
\begin{aligned}
\mathcal{X}_{T, \rho}:=\left\{(f, Q, L) \in \mathcal{X}_{T} \mid\|Q\|_{L^{\infty}\left(0, T ; L^{2}\right)}\right. & \leq \rho_{Q}, \quad\|f(t)\|_{L^{2}(Z)} \leq \rho_{f} \cdot e^{\gamma_{f} t} \\
\|L(t)\|_{W^{1,2}\left(\mathbb{R}^{n}\right)} & \left.\leq \rho_{L} \cdot\left(1+t^{-\frac{1}{2}}\right) \quad \text { for a.e. } t \in[0, T]\right\}
\end{aligned}
$$

supplied with the metric

$$
\begin{aligned}
d^{\mathcal{X}}: \quad \mathcal{X}_{T, \rho} \times \mathcal{X}_{T, \rho} & \longrightarrow \mathbb{R}, \\
\left(\left(f_{1}, Q_{1}, L_{1}\right), \quad\left(f_{2}, Q_{2}, L_{2}\right)\right) \longmapsto & \sup _{t \in[0, T]}\left(d^{f}\left(f_{1}(t), f_{2}(t)\right)+\left\|Q_{1}(t)-Q_{2}(t)\right\|_{L^{2}\left(\mathbb{R}^{n} \times \mathbb{S}^{n-1}\right)}+\right. \\
& \left.\left\|L_{1}(t)-L_{2}(t)\right\|_{L^{2}\left(\mathbb{R}^{n}\right)}\right)+ \\
& \operatorname{ess~sup}_{t} \frac{\left\|L_{1}(t)-L_{2}(t)\right\|_{W^{1,2}\left(\mathbb{R}^{n}\right)}}{1+t^{-1 / 2}} .
\end{aligned}
$$

Lemma 7.1 states $\mathcal{L} \mathcal{X}_{T, \rho} \subseteq \mathcal{X}_{T, \rho}$. Due to assumption (v), the same arguments as for Lemma 5.25 imply that $\left\{|f(t)|^{2} \mid(f, Q, L) \in \mathcal{L} \mathcal{X}_{T, \rho}, t \in[0, T]\right\} \subseteq L^{1}(Z)$ is tight. Due to Lemma 5.6, the closed convex hull of $\left\{f(t) \mid(f, Q, L) \in \mathcal{L} \mathcal{X}_{T, \rho}, t \in[0, T]\right\} \subseteq L^{2}(Z)$ is complete w.r.t. $d^{f}$. Hence the closed convex hull of $\mathcal{L} \mathcal{X}_{T, \rho}$ is complete w.r.t. $d^{\mathcal{X}}$.

Moreover $\mathcal{L}$ is Lipschitz continuous w.r.t. $d^{\mathcal{X}}$. Indeed, in combination with assumptions (ii),(iv) for the auxiliary radius $\max \left\{\rho_{f} \cdot e^{\gamma_{f} T}, \rho_{Q}, \rho_{L}\right\}$, Corollaries 5.28, 6.2 and 6.6 imply for any $\left(\widetilde{f}_{1}, \widetilde{Q}_{1}, \widetilde{L}_{1}\right),\left(\widetilde{f}_{2}, \widetilde{Q}_{2}, \widetilde{L}_{2}\right) \in \mathcal{X}_{T, \rho}$ and $\left(f_{j}, Q_{j}, L_{j}\right):=\mathcal{L}\left(\widetilde{f}_{j}, \widetilde{Q}_{j}, \widetilde{L}_{j}\right), j=1,2$,

$$
\begin{aligned}
& d^{f}\left(f_{1}(t), f_{2}(t)\right) \leq \operatorname{const}\left(\gamma_{f}, \rho_{f}, \rho_{Q}, \rho_{L}\right) \cdot \int_{0}^{t} \Lambda_{\rho}\left(d^{f}\left(\widetilde{f}_{1}(s), \widetilde{f}_{2}(s)\right)+\left\|\widetilde{Q}_{1}(s)-\widetilde{Q}_{2}(s)\right\|_{L^{2}}+\right. \\
&\left.\left\|\widetilde{L}_{1}(s)-\widetilde{L}_{2}(s)\right\|_{W^{1,2}}\right) d s \\
& \leq \operatorname{const}\left(\gamma_{f}, \rho_{f}, \rho_{Q}, \rho_{L}\right) \cdot \int_{0}^{t} \Lambda_{\rho} d^{\mathcal{X}}\left(\left(\widetilde{f}_{1}, \widetilde{Q}_{1}, \widetilde{L}_{1}\right),\left(\widetilde{f}_{2}, \widetilde{Q}_{2}, \widetilde{L}_{2}\right)\right)\left(1+s^{-\frac{1}{2}}\right) d s \\
& \leq \operatorname{const}\left(\gamma_{f}, \rho_{f}, \rho_{Q}, \rho_{L}\right) \cdot(t+\sqrt{t}) \cdot d^{\mathcal{X}}\left(\left(\widetilde{f}_{1}, \widetilde{Q}_{1}, \widetilde{L}_{1}\right),\left(\widetilde{f}_{2}, \widetilde{Q}_{2}, \widetilde{L}_{2}\right)\right) \\
&\left\|Q_{1}(t)-Q_{2}(t)\right\|_{L^{2}} \leq \operatorname{const}\left(\gamma_{f}, \rho_{f}, \rho_{Q}, \rho_{L}\right) \cdot(t+\sqrt{t}) \cdot d^{\mathcal{X}}\left(\left(\widetilde{f}_{1}, \widetilde{Q}_{1}, \widetilde{L}_{1}\right),\left(\widetilde{f}_{2}, \widetilde{Q}_{2}, \widetilde{L}_{2}\right)\right) \\
&\left\|L_{1}(t)-L_{2}(t)\right\|_{L^{2}} \leq \operatorname{const}\left(\gamma_{f}, \rho_{f}, \rho_{Q}, \rho_{L}, \kappa_{L}\right) \cdot \int_{0}^{t} \Lambda_{\rho}\left(d^{f}\left(\widetilde{f}_{1}(s), \widetilde{f}_{2}(s)\right)+\left\|\widetilde{Q}_{1}(s)-\widetilde{Q}_{2}(s)\right\|_{L^{2}}\right. \\
&\left.\quad+\left\|L_{1}(s)-L_{2}(s)\right\|_{L^{2}\left(\mathbb{R}^{n}\right)}\right) d s \\
&\left\|L_{1}(t)-L_{2}(t)\right\|_{L^{2}} \leq \operatorname{const}\left(\gamma_{f}, \rho_{f}, \rho_{Q}, \rho_{L}, \kappa_{L}, T\right) \cdot t \cdot d^{\mathcal{X}}\left(\left(\widetilde{f}_{1}, \widetilde{Q}_{1}, \widetilde{L}_{1}\right),\left(\widetilde{f}_{2}, \widetilde{Q}_{2}, \widetilde{L}_{2}\right)\right)
\end{aligned}
$$

with the last estimate being concluded from Gronwall's inequality. Moreover the spatial gradients of $L_{1}(t)-L_{2}(t)$ are bounded in the sense that

$$
\begin{aligned}
& \left\|\nabla_{\mathbf{x}}\left(L_{1}(t)-L_{2}(t)\right)\right\|_{L^{2}} \\
\leq & \operatorname{const}\left(\kappa_{L}, n\right) \sqrt{t} \cdot \sup _{s \in[0, t]} \Lambda_{\rho} \cdot\left(d^{f}\left(\widetilde{f}_{1}(s), \widetilde{f}_{2}(s)\right)+\left\|\widetilde{Q}_{1}(s)-\widetilde{Q}_{2}(s)\right\|_{L^{2}}+\left\|L_{1}(s)-L_{2}(s)\right\|_{L^{2}}\right) \\
\leq & \operatorname{const}\left(\rho_{f}, \rho_{Q}, \rho_{L}, \kappa_{L}, n, T\right) \cdot \sqrt{t} \cdot d^{\mathcal{X}}\left(\left(\widetilde{f}_{1}, \widetilde{Q}_{1}, \widetilde{L}_{1}\right),\left(\widetilde{f}_{2}, \widetilde{Q}_{2}, \widetilde{L}_{2}\right)\right),
\end{aligned}
$$


i.e. $\frac{\left\|L_{1}(t)-L_{2}(t)\right\|_{W^{1,2}}}{1+t^{-1 / 2}} \leq \operatorname{const}\left(\gamma_{f}, \rho_{f}, \rho_{Q}, \rho_{L}, \kappa_{L}, n, T\right) \cdot t \cdot d^{\mathcal{X}}\left(\left(\widetilde{f}_{1}, \widetilde{Q}_{1}, \widetilde{L}_{1}\right),\left(\widetilde{f}_{2}, \widetilde{Q}_{2}, \widetilde{L}_{2}\right)\right)$. Hence we obtain for every $t \in] 0, T]$

$$
\begin{aligned}
& \sup _{s \in] 0, t]}\left(d^{f}\left(f_{1}(s), f_{2}(s)\right)+\left\|Q_{1}(s)-Q_{2}(s)\right\|_{L^{2}}+\left\|L_{1}(s)-L_{2}(s)\right\|_{L^{2}}+\frac{\left\|L_{1}(s)-L_{2}(s)\right\|_{W^{1,2}}}{1+s^{-1 / 2}}\right) \\
& \quad \leq \operatorname{const}\left(\gamma_{f}, \rho_{f}, \rho_{Q}, \rho_{L}, \kappa_{L}, n, T\right) \cdot \sqrt{t} \cdot d^{\mathcal{X}}\left(\left(\widetilde{f}_{1}, \widetilde{Q}_{1}, \widetilde{L}_{1}\right),\left(\widetilde{f}_{2}, \widetilde{Q}_{2}, \widetilde{L}_{2}\right)\right) .
\end{aligned}
$$

Now choose $\left.\left.\tau=\tau\left(\gamma_{f}, \rho_{f}, \rho_{Q}, \rho_{L}, \kappa_{L}, n, T\right) \in\right] 0, T\right]$ sufficiently small with

$$
\operatorname{const}\left(\gamma_{f}, \rho_{f}, \rho_{Q}, \rho_{L}, \kappa_{L}, n, T\right) \cdot \sqrt{\tau} \leq \frac{1}{2} .
$$

If we now restrict our considerations to the subinterval $[0, \tau]$ and the related subset $\mathcal{X}_{\tau, \rho}$ (with the same parameters $\gamma_{f}, \rho_{f}, \rho_{Q}, \rho_{L}, \kappa_{L}$ though), then $\mathcal{L}: \mathcal{X}_{\tau, \rho} \longrightarrow \mathcal{X}_{\tau, \rho}$ proves to be a strict contraction and Banach's fixed point theorem provides a unique fixed point $\left(f_{1}, Q_{1}, L_{1}\right)$ which solves the given system in $[0, \tau]$ uniquely.

This step is now repeated in the subinterval $[\tau, 2 \tau]$, the initial states $f_{1}(\tau), Q_{1}(\tau), L_{1}(\tau)$ and the same parameters $\gamma_{f}, \rho_{Q}, \rho_{L}, \kappa_{L}$, but the modification $\rho_{f} \leftarrow \rho_{f} \cdot e^{\gamma_{f} \tau}$. In particular the same estimates ensure the strict contraction property etc. After finitely many steps (each of which has the same step size $\tau$ ) we obtain the unique solution in the whole time interval $[0, T]-$ as claimed in Theorem 3.2.

\subsection{Proof of Proposition 3.3 about Lipschitz continuous dependence of solutions on data for $Z \stackrel{\text { Def. }}{=} \mathbb{R}^{n} \times V \times \mathbb{R}^{d}$}

For the upper bound $R$ of initial states given, the a priori estimates presented in the proof of Lemma 7.1 provide constants $\gamma_{f}, \rho_{f}, \rho_{Q}, \rho_{L}>0$ depending on $R, T$ and coefficient constants in assumptions (i) - (ii) of Theorem 3.2 such that the following estimates hold for $j=1,2$ and every $t \in[0, T]$

$$
\begin{cases}\left\|f^{(j)}(t)\right\|_{L^{2}(Z)} & \leq \rho_{f} \cdot e^{\gamma_{f} \cdot t} \\ \left\|Q^{(j)}(t)\right\|_{L^{2}\left(\mathbb{R}^{n} \times \mathbb{S}^{n-1}\right)} & \leq \rho_{Q} \\ \left\|L^{(j)}(t)\right\|_{L^{2}\left(\mathbb{R}^{n}\right)} & \leq \rho_{L} \\ \left\|L^{(j)}(t)\right\|_{W^{1,2}\left(\mathbb{R}^{n}\right)} & \leq \rho_{L} \cdot\left(1+t^{-\frac{1}{2}}\right)\end{cases}
$$

This leads to a joint Lipschitz constant $\Lambda_{0}=\Lambda_{0}(R, T)>0$ for $\mathcal{R}^{(j)}$ and $\mathcal{G}^{(j)}, \mathcal{U}^{(j)}, \mathcal{W}^{(j)}, \mathcal{T}^{(j)}$ $(j=1,2)$ (in the sense of assumption (iv) in Theorem 3.2).

Corollary 5.28 provides a constant $C>0$ (depending on $R$ and the joint bounds of coefficients) such that for every $t \in[0, T]$

$$
\begin{aligned}
d^{f}\left(f^{(1)}(t), f^{(2)}(t)\right) \leq & \left(d^{f}\left(f_{0}^{(1)}, f_{0}^{(2)}\right)+\right. \\
C \cdot \int_{0}^{t} & \|\left.\left(\mathcal{G}^{(1)}, \mathcal{U}^{(1)}, \mathcal{W}^{(1)}\right)\right|_{\left(s, f^{(1)}(s), Q^{(1)}(s), L^{(1)}(s)\right)}- \\
& \left.\left.\left(\mathcal{G}^{(2)}, \mathcal{U}^{(2)}, \mathcal{W}^{(2)}\right)\right|_{\left(s, f^{(2)}(s), Q^{(2)}(s), L^{(2)}(s)\right)} \|_{L^{2}\left(Z, \mathbb{R}^{d+2}\right)} d s\right) \cdot e^{C t} .
\end{aligned}
$$


Now $\Lambda_{0}$-Lipschitz continuity of the coefficient functions and Minkowski inequality imply

$$
\begin{aligned}
& d^{f}\left(f^{(1)}(t), f^{(2)}(t)\right) \\
& \leq \quad\left(d^{f}\left(f_{0}^{(1)}, f_{0}^{(2)}\right)+\right. \\
& \quad C \cdot t \cdot \sup _{[0, T] \times L^{2}(Z) \times L^{2}\left(\mathbb{R}^{n} \times \mathbb{S}^{n-1}\right) \times W^{1,2}\left(\mathbb{R}^{n}\right)}\left\|\left(\mathcal{G}^{(1)}, \mathcal{U}^{(1)}, \mathcal{W}^{(1)}\right)-\left(\mathcal{G}^{(2)}, \mathcal{U}^{(2)}, \mathcal{W}^{(2)}\right)\right\|_{L^{2}}+ \\
& \left.\quad C \cdot \int_{0}^{t} \Lambda_{0} \cdot\left(d^{f}\left(f^{(1)}, f^{(2)}\right)+\left\|Q^{(1)}-Q^{(2)}\right\|_{L^{2}}+\left\|L^{(1)}-L^{(2)}\right\|_{W^{1,2}}\right) d s\right) \cdot e^{C t} .
\end{aligned}
$$

For the same reason, we conclude from Corollary 6.2 for every $t \in[0, T]$

$$
\begin{aligned}
& \left\|Q^{(1)}(t)-Q^{(2)}(t)\right\|_{L^{2}\left(\mathbb{R}^{n} \times \mathbb{S}^{n-1}\right)} \\
& \leq \quad\left\|Q_{0}^{(1)}-Q_{0}^{(2)}\right\|_{L^{2}\left(\mathbb{R}^{n} \times \mathbb{S}^{n-1}\right)} \\
& \quad+\int_{0}^{t}\left\|\left.\mathcal{T}^{(1)}\right|_{\left(s, f^{(1)}(s), Q^{(1)}(s), L^{(1)}(s)\right)}-\left.\mathcal{T}^{(2)}\right|_{\left(s, f^{(2)}(s), Q^{(2)}(s), L^{(2)}(s)\right.}\right\|_{L^{2}\left(\mathbb{R}^{n} \times \mathbb{S}^{n-1}\right)} d s \\
& \leq \quad\left\|Q_{0}^{(1)}-Q_{0}^{(2)}\right\|_{L^{2}\left(\mathbb{R}^{n} \times \mathbb{S}^{n-1}\right)}\left\|\sup ^{(1)}-\mathcal{T}^{(2)}\right\|_{L^{2}\left(\mathbb{R}^{n} \times \mathbb{S}^{n-1}\right)} \\
& \quad+t \cdot\left[\begin{array}{l}
{[0, T] \times L^{2}(Z) \times L^{2}\left(\mathbb{R}^{n} \times \mathbb{S}^{n-1}\right) \times W^{1,2}\left(\mathbb{R}^{n}\right)} \\
\quad+\Lambda_{0} \cdot \int_{0}^{t}\left(d^{f}\left(f^{(1)}(s), f^{(2)}(s)\right)+\left\|Q^{(1)}(s)-Q^{(2)}(s)\right\|_{L^{2}}+\left\|L^{(1)}(s)-L^{(2)}(s)\right\|_{W^{1,2}}\right) d s .
\end{array}\right.
\end{aligned}
$$

The corresponding estimates for $L^{(1)}(t)-L^{(2)}(t)$ result from Corollary 6.6, i.e., first,

$$
\begin{aligned}
& \left\|L^{(1)}(t)-L^{(2)}(t)\right\|_{L^{2}\left(\mathbb{R}^{n}\right)} \\
& \leq \quad\left\|L_{0}^{(1)}-L_{0}^{(2)}\right\|_{L^{2}\left(\mathbb{R}^{n}\right)} \\
& \quad+C \cdot \int_{0}^{t}\left\|\left.\mathcal{R}^{(1)}\right|_{\left(s, f^{(1)}(s), Q^{(1)}(s), L^{(1)}(s)\right)}-\left.\mathcal{R}^{(2)}\right|_{\left(s, f^{(2)}(s), Q^{(2)}(s), L^{(2)}(s)\right)}\right\|_{L^{2}\left(\mathbb{R}^{n}\right)} d s \\
& \quad \\
& \quad\left\|L_{0}^{(1)}-L_{0}^{(2)}\right\|_{L^{2}\left(\mathbb{R}^{n}\right)}+C t \cdot \sup \left\|\mathcal{R}^{(1)}-\mathcal{R}^{(2)}\right\|_{L^{2}\left(\mathbb{R}^{n}\right)} \\
& \quad+C \Lambda_{0} \cdot \int_{0}^{t}\left(d^{f}\left(f^{(1)}(s), f^{(2)}(s)\right)+\left\|Q^{(1)}(s)-Q^{(2)}(s)\right\|_{L^{2}}+\left\|L^{(1)}(s)-L^{(2)}(s)\right\|_{L^{2}}\right) d s
\end{aligned}
$$

and second,

$$
\begin{aligned}
\| & \nabla_{\mathbf{x}}\left(L^{(1)}(t)-L^{(2)}(t)\right) \|_{L^{2}\left(\mathbb{R}^{n}\right)} \\
\leq \quad & C \cdot\left(t^{-\frac{1}{2}} \cdot\left\|L_{0}^{(1)}-L_{0}^{(2)}\right\|_{L^{2}\left(\mathbb{R}^{n}\right)}+\right. \\
& \left.\quad \int_{0}^{t}(t-s)^{-\frac{1}{2}} \cdot\left\|\left.\mathcal{R}^{(1)}\right|_{\left(s, f^{(1)}(s), Q^{(1)}(s), L^{(1)}(s)\right)}-\left.\mathcal{R}^{(2)}\right|_{\left(s, f^{(2)}(s), Q^{(2)}(s), L^{(2)}(s)\right)}\right\|_{L^{2}\left(\mathbb{R}^{n}\right)} d s\right) \\
\leq \quad C \cdot & \left(t^{-\frac{1}{2}} \cdot\left\|L_{0}^{(1)}-L_{0}^{(2)}\right\|_{L^{2}\left(\mathbb{R}^{n}\right)}+2 \sqrt{t} \cdot \sup \left\|\mathcal{R}^{(1)}-\mathcal{R}^{(2)}\right\|_{L^{2}\left(\mathbb{R}^{n}\right)}+\right. \\
& \left.\Lambda_{0} \cdot \int_{0}^{t}(t-s)^{-\frac{1}{2}} \cdot\left(d^{f}\left(f^{(1)}, f^{(2)}\right)+\left\|Q^{(1)}-Q^{(2)}\right\|_{L^{2}}+\left\|L^{(1)}-L^{(2)}\right\|_{L^{2}}\right) d s\right)
\end{aligned}
$$


Now we consider the auxiliary distance function $\phi:[0, T] \longrightarrow[0, \infty[$,

$$
\phi(t):=\sup _{s \in[0, t]}\left(d^{f}\left(f^{(1)}(s), f^{(2)}(s)\right)+\left\|Q^{(1)}(s)-Q^{(2)}(s)\right\|_{L^{2}\left(\mathbb{R}^{n} \times \mathbb{S}^{n-1}\right)}+\left\|L^{(1)}(s)-L^{(2)}(s)\right\|_{L^{2}}\right)
$$

Obviously, it is nondecreasing and so, we obtain for every $t \in] 0, T]$

$$
\begin{aligned}
& \left\|\nabla_{\mathbf{x}}\left(L^{(1)}(t)-L^{(2)}(t)\right)\right\|_{L^{2}\left(\mathbb{R}^{n}\right)} \\
& \leq C \cdot\left(t^{-\frac{1}{2}} \cdot\left\|L_{0}^{(1)}-L_{0}^{(2)}\right\|_{L^{2}\left(\mathbb{R}^{n}\right)}+2 \sqrt{t} \cdot \sup \left\|\mathcal{R}^{(1)}-\mathcal{R}^{(2)}\right\|_{L^{2}\left(\mathbb{R}^{n}\right)}+\right. \\
& \left.\quad \Lambda_{0} \cdot \phi(t) \cdot \int_{0}^{t}(t-s)^{-\frac{1}{2}} d s\right) \\
& =C \cdot\left(t^{-\frac{1}{2}} \cdot\left\|L_{0}^{(1)}-L_{0}^{(2)}\right\|_{L^{2}\left(\mathbb{R}^{n}\right)}+2 \sqrt{t} \cdot \sup \left\|\mathcal{R}^{(1)}-\mathcal{R}^{(2)}\right\|_{L^{2}\left(\mathbb{R}^{n}\right)}+2 \Lambda_{0} \sqrt{t} \cdot \phi(t)\right) .
\end{aligned}
$$

Hence the distance function $\psi:[0, T] \longrightarrow[0, \infty[$ of interest here satisfies the integral inequality for every $t \in] 0, T]$

$$
\begin{aligned}
\psi(t) \stackrel{\text { Def. }}{=} & d^{f}\left(f^{(1)}(t)-f^{(2)}(t)\right)+\left\|Q^{(1)}(t)-Q^{(2)}(t)\right\|_{L^{2}\left(\mathbb{R}^{n} \times \mathbb{S}^{n-1}\right)}+\left\|L^{(1)}(t)-L^{(2)}(t)\right\|_{L^{2}\left(\mathbb{R}^{n}\right)} \\
\leq & d^{f}\left(f_{0}^{(1)}, f_{0}^{(2)}\right) \cdot e^{C t}+\left\|Q_{0}^{(1)}-Q_{0}^{(2)}\right\|_{L^{2}\left(\mathbb{R}^{n} \times \mathbb{S}^{n-1}\right)}+\left\|L_{0}^{(1)}-L_{0}^{(2)}\right\|_{L^{2}\left(\mathbb{R}^{n}\right)} \\
& +C t e^{C t} \cdot \sup \left\|\left(\mathcal{G}^{(1)}, \mathcal{U}^{(1)}, \mathcal{W}^{(1)}, \mathcal{T}^{(1)}, \mathcal{R}^{(1)}\right)-\left(\mathcal{G}^{(2)}, \mathcal{U}^{(2)}, \mathcal{W}^{(2)}, \mathcal{T}^{(2)}, \mathcal{R}^{(2)}\right)\right\|_{L^{2}} \\
& +3 \Lambda_{0} C e^{C t} \cdot \int_{0}^{t}\left(d^{f}\left(f^{(1)}, f^{(2)}\right)+\left\|Q^{(1)}-Q^{(2)}\right\|_{L^{2}}+\left\|L^{(1)}-L^{(2)}\right\|_{L^{2}}\right) d s \\
& +2 \Lambda_{0} C e^{C t} \cdot \int_{0}^{t}\left\|\nabla_{\mathbf{x}}\left(L^{(1)}(s)-L^{(2)}(s)\right)\right\|_{L^{2}} d s \\
\leq & \psi(0) \cdot e^{C t} \\
& +C t e^{C t} \cdot \sup \left\|\left(\mathcal{G}^{(1)}, \mathcal{U}^{(1)}, \mathcal{W}^{(1)}, \mathcal{T}^{(1)}, \mathcal{R}^{(1)}\right)-\left(\mathcal{G}^{(2)}, \mathcal{U}^{(2)}, \mathcal{W}^{(2)}, \mathcal{T}^{(2)}, \mathcal{R}^{(2)}\right)\right\|_{L^{2}} \\
& +3 \Lambda_{0} C e^{C t} \cdot \int_{0}^{t} \psi(s) d s \\
& +2 \Lambda_{0} C^{2} e^{C t} \cdot\left(2 \sqrt{t} \cdot\left\|L_{0}^{(1)}-L_{0}^{(2)}\right\|_{L^{2}}+3 t^{\frac{3}{2}} \cdot \sup \left\|\mathcal{R}^{(1)}-\mathcal{R}^{(2)}\right\|_{L^{2}}+2 \Lambda_{0} \int_{0}^{t} \phi(s) \sqrt{s} d s\right) .
\end{aligned}
$$

We keep the notation $C$ for each constant depending only on $R, T$ and the bounds of the coefficients (stated in assumptions (i) - (iv) of Theorem 3.2). Moreover set

$$
\Delta_{\infty}:=\sup \left\|\left(\mathcal{G}^{(1)}, \mathcal{U}^{(1)}, \mathcal{W}^{(1)}, \mathcal{T}^{(1)}, \mathcal{R}^{(1)}\right)-\left(\mathcal{G}^{(2)}, \mathcal{U}^{(2)}, \mathcal{W}^{(2)}, \mathcal{T}^{(2)}, \mathcal{R}^{(2)}\right)\right\|_{L^{2}} .
$$

Then $\phi(t)=\sup _{0 \leq s \leq t} \psi(s)$ implies the estimate for every $\left.\left.t \in\right] 0, T\right]$

$$
\begin{aligned}
\phi(t) & \leq \phi(0) \cdot e^{C t}(1+C \sqrt{t})+C t e^{C t} \cdot \Delta_{\infty}+C \int_{0}^{t} \phi(s) \cdot(1+\sqrt{s}) d s \\
& \leq \phi(0) \cdot e^{C(t+\sqrt{t})}+C t e^{C t} \cdot \Delta_{\infty}+C \int_{0}^{t} \phi(s) d s \\
\Longrightarrow \phi(t) & \leq\left(\phi(0) \cdot e^{C \sqrt{t}}+C t \cdot \Delta_{\infty}\right) \cdot e^{2 C t}
\end{aligned}
$$

due to Gronwall's inequality and thus, $\psi \leq \phi$ leads to the claim (22). 


\subsection{Proof of Corollary 3.4}

By assumption, $Y \subsetneq \mathbb{R}^{d}$ is now an open subset with bounded Lipschitz boundary. As before, we set $Z \stackrel{\text { Def. }}{=} \mathbb{R}^{n} \times V \times Y$.

We consider the hypothesis of the boundary $\partial Y \subseteq \mathbb{R}^{d}$ being Lipschitz and compact for two technical reasons. First every Sobolev function $W^{1, \infty}(Y)$ is (globally) Lipschitz continuous and so, it can be extended continuously to the topological closure $\bar{Y} \subset \mathbb{R}^{d}$. Second, there exists a continuous extension operator for Sobolev functions with some properties which are usually not mentioned explicitly in the literature. Indeed, the proofs in $[14, \S 6.4]$ reveal for Lipschitz boundaries (similarly to what can be found in [13, Theorem 9.7] for open sets with $C^{1}$ boundaries):

\section{Lemma 7.2 (Extension of Sobolev functions - independent of $k, p$ )}

Let $\Omega \subset \mathbb{R}^{d}$ be an open set with compact Lipschitz boundary. Then there exists a linear extension operator $\mathcal{E}: L_{\mathrm{loc}}^{1}(\Omega) \longrightarrow L_{\mathrm{loc}}^{1}\left(\mathbb{R}^{d}\right)$ which, in addition to the standard condition on extensions, i.e. $\left.(\mathcal{E} u)\right|_{\Omega}=u$ for all $u \in L_{\mathrm{loc}}^{1}(\Omega)$, satisfies for any $k \in \mathbb{N}, p \in[1, \infty]$ and $u \in W^{k, p}(\Omega)$

$$
\begin{aligned}
\mathcal{E}\left(W^{k, p}(\Omega)\right) & \subset W^{k, p}\left(\mathbb{R}^{d}\right), \\
\|\mathcal{E} u\|_{L^{p}\left(\mathbb{R}^{d}\right)} & \leq \operatorname{const}(\Omega, k, p) \cdot\|u\|_{L^{p}(\Omega)}, \\
\|\mathcal{E} u\|_{W^{k, p}\left(\mathbb{R}^{d}\right)} & \leq \operatorname{const}(\Omega, k, p) \cdot\|u\|_{W^{k, p}(\Omega)} .
\end{aligned}
$$

This extension operator $\mathcal{E}$ will be applied to the coefficient functions $\mathcal{G}$ and $\mathcal{U}$ for extending the initial value problem (20) from $Z \stackrel{\text { Def. }}{=} \mathbb{R}^{n} \times V \times Y$ to the "whole" space $\mathbb{R}^{n} \times V \times \mathbb{R}^{d}$, which was considered in Theorem 3.2 and Corollary 3.3. As an abbreviation, define the linear operator

$$
\mathcal{E}_{\mathbf{y}}: L_{\mathrm{loc}}^{1}\left(\mathbb{R}^{n} \times V \times Y\right) \longrightarrow L_{\mathrm{loc}}^{1}\left(\mathbb{R}^{n} \times V \times \mathbb{R}^{d}\right)
$$

by $\left(\mathcal{E}_{\mathbf{y}} u\right)(\mathbf{x}, \mathbf{v}, \mathbf{y}):=\mathcal{E}(u(\mathbf{x}, \mathbf{v}, \cdot))(\mathbf{y})$ for $u \in L_{\text {loc }}^{1}\left(\mathbb{R}^{n} \times V \times Y\right)$ and $(\mathbf{x}, \mathbf{v}, \mathbf{y}) \in \mathbb{R}^{n} \times V \times \mathbb{R}^{d}$.

Indeed, for every $t \in[0, T], f \in L^{2}(Z), Q \in L^{2}\left(\mathbb{R}^{n} \times \mathbb{S}^{n-1}\right)$ and $L \in W^{1,2}\left(\mathbb{R}^{n}\right)$, the function $\mathcal{G}(t, f, Q, L) \in L^{\infty}\left(Z, \mathbb{R}^{d}\right) \cap L^{2}$ has its weak partial derivative w.r.t. $\mathbf{y}$ in $L^{\infty}\left(Z, \mathbb{R}^{d \times d}\right)$. Hence, for Lebesgue-almost every $\mathbf{x} \in \mathbb{R}^{n}$ and $\mathbf{v} \in V$,

$$
Y \longrightarrow \mathbb{R}^{d}, \quad \mathbf{y} \longmapsto \mathcal{G}(t, f, Q, L)(\mathbf{x}, \mathbf{v}, \mathbf{y})
$$

belongs to $W^{1, \infty}\left(Y, \mathbb{R}^{d}\right)$ and so, $\mathcal{E}$ provides an extension to $\mathbb{R}^{d}$ denoted by

$$
\mathbb{R}^{d} \longrightarrow \mathbb{R}^{d}, \quad \mathbf{y} \longmapsto(\mathcal{E} \circ \mathcal{G}(t, f, Q, L)(\mathbf{x}, \mathbf{v}, \cdot))(\mathbf{y})
$$

with

$$
\begin{cases}\|\mathcal{E} \circ \mathcal{G}(t, f, Q, L)(\mathbf{x}, \mathbf{v}, \cdot)\|_{L^{2}\left(\mathbb{R}^{d}\right)} & \leq \text { const } \cdot\|\mathcal{G}(t, f, Q, L)(\mathbf{x}, \mathbf{v}, \cdot)\|_{L^{2}(Y)} \\ \|\mathcal{E} \circ \mathcal{G}(t, f, Q, L)(\mathbf{x}, \mathbf{v}, \cdot)\|_{W^{1, \infty}\left(\mathbb{R}^{d}\right)} & \leq \mathrm{const} \cdot\|\mathcal{G}(t, f, Q, L)(\mathbf{x}, \mathbf{v}, \cdot)\|_{W^{1, \infty}(Y)}\end{cases}
$$


Thus, the composition $\mathcal{E}_{\mathbf{y}} \circ \mathcal{G}(t, f, Q, L)$ belongs to $L^{2}\left(\mathbb{R}^{n} \times V \times \mathbb{R}^{d}, \mathbb{R}^{d}\right) \cap L^{\infty}$ and has its weak derivative w.r.t. $\mathbf{y}$ in $L^{\infty}\left(\mathbb{R}^{n} \times V \times \mathbb{R}^{d}, \mathbb{R}^{d \times d}\right)$. Moreover all related norms have upper bounds in terms of the corresponding norms of $\mathcal{G}(t, f, Q, L)$.

Applying the same arguments to $\mathcal{E}_{\mathbf{y}} \circ \mathcal{U}(t, f, Q, L) \in L^{2}\left(\mathbb{R}^{n} \times V \times \mathbb{R}^{d}\right) \cap L^{\infty}$ and using the zero expansion of $\mathcal{W}(t, f, Q, L) \in L^{2}(Z)$ to $\mathbb{R}^{n} \times V \times \mathbb{R}^{d}$, we now have the tools for solving the following auxiliary problem for $\breve{f}:[0, T] \longrightarrow L^{2}\left(\mathbb{R}^{n} \times V \times \mathbb{R}^{d}\right), \breve{Q}:[0, T] \longrightarrow L^{2}\left(\mathbb{R}^{n} \times \mathbb{S}^{n-1}\right)$, $\breve{L}:] 0, T] \longrightarrow W^{1,2}\left(\mathbb{R}^{n}\right)$

$$
\left\{\begin{aligned}
\partial_{t} \breve{f}+\nabla_{\mathbf{x}} \cdot(\mathbf{v} \breve{f})+\nabla_{\mathbf{y}} \cdot\left(\mathcal{E}_{\mathbf{y}} \circ \mathcal{G}\left(t,\left.\breve{f}\right|_{Z}, \breve{Q}, \breve{L}\right) \breve{f}\right) & =\mathcal{E}_{\mathbf{y}} \circ \mathcal{U}\left(t,\left.\breve{f}\right|_{Z}, \breve{Q}, \breve{L}\right) \breve{f}+\mathcal{W}\left(t,\left.\breve{f}\right|_{Z}, \breve{Q}, \breve{L}\right) \\
\partial_{t} \breve{Q} & =\mathcal{T}\left(t,\left.\breve{f}\right|_{Z}, \breve{Q}, \breve{L}\right) \\
\partial_{t} \breve{L} & =\kappa_{L} \Delta_{\mathbf{x}} L+\mathcal{R}\left(t,\left.\breve{f}\right|_{Z}, \breve{Q}, \breve{L}\right) .
\end{aligned}\right.
$$

Indeed, all assumptions of Theorem 3.2 are satisfied and so, there exists a unique weak solution $\left.\left.\breve{f}:[0, T] \longrightarrow L^{2}\left(\mathbb{R}^{n} \times V \times \mathbb{R}^{d}\right), \breve{Q}:[0, T] \longrightarrow L^{2}\left(\mathbb{R}^{n} \times \mathbb{S}^{n-1}\right), \breve{L}:\right] 0, T\right] \longrightarrow W^{1,2}\left(\mathbb{R}^{n}\right)$ for any initial states $\breve{f}_{0} \in L^{2}\left(\mathbb{R}^{n} \times V \times \mathbb{R}^{d}\right), \breve{Q}_{0} \in L^{2}\left(\mathbb{R}^{n} \times \mathbb{S}^{n-1}\right), \breve{L}_{0} \in L^{2}\left(\mathbb{R}^{n}\right)$ given.

Considering smooth test functions with compact support in $Z \stackrel{\text { Def. }}{=} \mathbb{R}^{n} \times V \times Y$ (rather than $\mathbb{R}^{n} \times V \times \mathbb{R}^{d}$ as permitted in the auxiliary problem), we obtain immediately that the restrictions $f(t):=\left.\breve{f}(t)\right|_{Z} \in L^{2}(Z), t \in[0, T]$, provide a weak solution to the original problem (20) on $Z$. The Lipschitz continuous dependence on given data (in the sense of estimate (22)) remains to be proved because it implies uniqueness.

All statements about the extended problem on the "whole space" $\mathbb{R}^{n} \times V \times \mathbb{R}^{n}$ use the metric

$$
\begin{array}{r}
\breve{d^{f}}(\breve{f}, \breve{g}) \stackrel{\text { Def. }}{=} \sup \left\{\int_{\mathbb{R}^{n} \times V \times \mathbb{R}^{d}} \varphi \cdot(\breve{f}-\breve{g}) d \mathbf{z} \mid \varphi \in C_{c}^{0}\left(\mathbb{R}^{n} \times V \times \mathbb{R}^{d}\right), \quad \partial_{\mathbf{y}} \varphi \in L^{\infty},\right. \\
\left.\|\varphi\|_{L^{2}} \leq 1, \quad\|\varphi\|_{L^{\infty}} \leq 1, \quad\left\|\partial_{\mathbf{y}} \varphi\right\|_{L^{\infty}} \leq 1\right\},
\end{array}
$$

but the estimate of interest now refers to the smaller domain $Z \stackrel{\text { Def. }}{=} \mathbb{R}^{n} \times V \times Y$ and its related distance of $\breve{f}, \breve{g} \in L^{2}\left(\mathbb{R}^{n} \times V \times \mathbb{R}^{d}\right)$, i.e.

$$
\begin{aligned}
& d^{f}(\breve{f}, \breve{g}) \stackrel{\text { Def. }}{=} \sup \left\{\int_{Z} \varphi \cdot(\breve{f}-\breve{g}) d \mathbf{z} \mid \varphi \in C_{c}^{0}(Z), \quad \partial_{\mathbf{y}} \varphi \in L^{\infty}\left(Z, \mathbb{R}^{d}\right),\right. \\
&\left.\|\varphi\|_{L^{2}} \leq 1,\|\varphi\|_{L^{\infty}} \leq 1,\left\|\partial_{\mathbf{y}} \varphi\right\|_{L^{\infty}} \leq 1\right\},
\end{aligned}
$$

which differs in the domain of the test functions. In particular, $d^{f}(\breve{f}, \breve{g})=d^{f}\left(\left.\breve{f}\right|_{Z},\left.\breve{g}\right|_{Z}\right)$.

Definition 7.3 ([7, Definition 4.1.1]) Let $K$ be any nonempty subset of $\mathbb{R}^{d}$ and $\xi \in \bar{K}$.

$$
T_{K}(\xi):=\left\{\mathbf{w} \in \mathbb{R}^{d} \mid \liminf _{h \downarrow 0} \frac{1}{h} \cdot \operatorname{dist}(\xi+h \cdot \mathbf{w}, K)=0\right\}
$$

is called the contingent cone of $K$ in $\xi$ (in the sense of Bouligand). 


\section{Proposition 7.4 (Characterising invariant sets for ordinary diff. equations)}

Suppose $\widetilde{\mathbf{g}}:[0, T] \times \mathbb{R}^{d} \longrightarrow \mathbb{R}^{d}$ to be an integrably bounded Carathéodory function, i.e.,

(i) for every $\xi \in \mathbb{R}^{d}, \widetilde{\mathbf{g}}(\cdot, \xi):[0, T] \longrightarrow \mathbb{R}^{d}$ is Lebesgue-measurable,

(ii) for Lebesgue-almost every $t \in[0, T], \widetilde{\mathbf{g}}(t, \cdot): \mathbb{R}^{d} \longrightarrow \mathbb{R}^{d}$ is continuous,

(iii) there is $\mu \in L^{1}([0, T])$ with $|\widetilde{\mathbf{g}}(t, \xi)| \leq \mu(t)$ for almost every $t \in[0, T]$ and all $\xi \in \mathbb{R}^{d}$.

For any nonempty closed set $K \subset \mathbb{R}^{d}$, the following statements are equivalent:

(a) In each point of $K$, there starts an absolutely continuous solution $\eta:[0, T] \longrightarrow \mathbb{R}^{d}$ of $\eta^{\prime}=\widetilde{\mathbf{g}}(\cdot, \eta)$ a.e. in $[0, T]$ satisfying the state constraint $\eta(t) \in K$ for every $t \in[0, T]$.

(b) For Lebesgue-almost every $t \in[0, T]$, the vector $\widetilde{\mathbf{g}}(t, \xi) \in \mathbb{R}^{d}$ belongs to the contingent cone $T_{K}(\xi)$ of $K$ in each point $\xi \in K$.

If, in addition, there exists some $\lambda \in L^{1}([0, T])$ such that for Lebesgue-almost all $t \in[0, T]$, the function $\widetilde{\mathbf{g}}(t, \cdot): \mathbb{R}^{d} \longrightarrow \mathbb{R}^{d}$ is $\lambda(t)$-Lipschitz continuous, then these statements are equivalent to

(c) For Lebesgue-almost every $t \in[0, T], \widetilde{\mathbf{g}}(t, \xi) \in \overline{\mathrm{co}} T_{K}(\xi)$ is satisfied for every $\xi \in K$.

Remark 7.5 Such a characterisation of flow-invariant subsets (in terms of tangent cones) was first introduced for autonomous differential equations by Nagumo [48] in 1942 and then often rediscovered independently. An extension to nonautonomous differential equations with Carathéodory right-hand side can be found in [62]. Generalisations to differential inclusions (via set-valued maps on the right-hand side) are known as viability theorems and have been investigated by several authors like Aubin, Cârjă, Deimling, Frankowska, Haddad et al. Their publications mostly differ from each other in the basic vector space, the assumptions about the right-hand side (of the differential inclusion) and the tangential criteria.

Here we use [26, Theorems 4.2] applied to a Carathéodory function (instead of a set-valued map) and [26, Theorems 4.7] in the Lipschitz continuous case.

On our way proving estimate (22) under the assumptions of Corollary 3.4, the basic idea is now to apply the gist of this invariance criterion to the subset $\bar{Z}=\mathbb{R}^{n} \times V \times \bar{Y}$.

For technical reasons (i.e., mollifying in a moment), fix any bounded open neighbourhood $\widehat{V} \subset \mathbb{R}^{n}$ of the compact set $V \stackrel{\text { Def. }}{=}\left[s_{1}, s_{2}\right] \cdot \mathbb{S}^{n-1} \subset \mathbb{R}^{n}$. The flow is induced by the nonautonomous vector field

$$
\begin{aligned}
{[0, T] \times \mathbb{R}^{n} \times \widehat{V} \times \mathbb{R}^{d} } & \longrightarrow \mathbb{R}^{n} \times \mathbb{R}^{n} \times \mathbb{R}^{d} \\
(t, \mathbf{x}, \mathbf{v}, \mathbf{y}) & \longmapsto\left(\mathbf{v}, 0, \widetilde{\mathbf{g}}(t)\left(\mathbf{x}, \operatorname{proj}_{V} \mathbf{v}, \mathbf{y}\right)\right)
\end{aligned}
$$

with the continuous function

$$
\widetilde{\mathbf{g}}: \quad[0, T] \longrightarrow L^{2}\left(\mathbb{R}^{n} \times \widehat{V} \times \mathbb{R}^{d}, \mathbb{R}^{d}\right), \quad t \longmapsto \mathcal{E}_{\mathbf{y}} \circ \mathcal{G}\left(t,\left.\breve{f}(t)\right|_{Z}, \breve{Q}(t), \breve{L}(t)\right) .
$$


Proposition 7.4, however, cannot be applied immediately because $\widetilde{\mathbf{g}}(t)$ is not continuous w.r.t. $\mathbf{x}, \mathbf{v}$ and so, the nonautonomous vector field indicated before is not Carathéodory in general. But the assumptions about $\mathcal{G}$ guarantee that for all $t \in[0, T]$ and every $\mathbf{x} \in \mathbb{R}^{n}, \mathbf{v} \in \widehat{V}$, the function $\widetilde{\mathbf{g}}(t)\left(\mathbf{x}, \operatorname{proj}_{V} \mathbf{v}, \cdot\right): \mathbb{R}^{d} \longrightarrow \mathbb{R}^{d}$ is Lipschitz continuous and, its Lipschitz constant is bounded independently of $t, \mathbf{x}, \mathbf{v}$.

The next step aims at smoothing the vector field and considers mollification w.r.t. $\mathbf{x}, \mathbf{v}$ only. As an important advantage, we "almost" preserve the tangential property specified in assumption (vi) of Corollary 3.4. Indeed, the contingent cone is now replaced by its closed convex hull, but then Lipschitz continuity enables us to apply Proposition 7.4.

Lemma 7.6 Let $\mathbf{g}: \mathbb{R}^{n} \times V \times \bar{Y} \longrightarrow \mathbb{R}^{d}$ be square Lebesgue integrable and essentially bounded with $\mathbf{g}(\mathbf{x}, \mathbf{v}, \cdot) \in W^{1, \infty}(\bar{Y}), \partial_{\mathbf{y}} \mathbf{g} \in L^{\infty}\left(\mathbb{R}^{n} \times V \times \bar{Y}\right)$ and $\mathbf{g}(\mathbf{x}, \mathbf{v}, \mathbf{y}) \in T_{Y}(\mathbf{y})$ for every $\mathbf{x}, \mathbf{v}, \mathbf{y}$. Consider any smooth Dirac sequences $\left(\rho_{k}\right)_{k \in \mathbb{N}},\left(\check{\rho}_{l}\right)_{l \in \mathbb{N}}$ with shrinking support in $\mathbb{B}_{1}(0) \subset \mathbb{R}^{n}$ and set for each $k, l \in \mathbb{N}$

$\mathbf{g}_{k, l}: \mathbb{R}^{n} \times V \times \bar{Y} \longrightarrow \mathbb{R}^{d}$

$$
(\mathbf{x}, \mathbf{v}, \mathbf{y}) \longmapsto\left(\mathbf{g} * \rho_{k}\right) * \check{\rho}_{l} \stackrel{\text { Def. }}{=} \int_{\mathbb{R}^{n}}\left(\int_{\mathbb{R}^{n}} \mathbf{g}\left(\widetilde{\mathbf{x}}, \operatorname{proj}_{V} \widetilde{\mathbf{v}}, \mathbf{y}\right) \cdot \rho_{k}(\mathbf{x}-\widetilde{\mathbf{x}}) d \widetilde{\mathbf{x}}\right) \cdot \check{\rho}_{l}(\mathbf{v}-\widetilde{\mathbf{v}}) d \widetilde{\mathbf{v}} .
$$

Then for all indices $k, l \in \mathbb{N}$ sufficiently large, the function $\mathbf{g}_{k, l}$ is Lipschitz continuous, bounded, square integrable and satisfies

$$
\left\{\begin{aligned}
\left\|\mathbf{g}_{k, l}\right\|_{L^{2}} & \leq\|\mathbf{g}\|_{L^{2}}, & & \left\|\mathbf{g}_{k, l}\right\|_{L^{\infty}} \leq\|\mathbf{g}\|_{L^{\infty}}, \\
\left\|\partial_{\mathbf{y}} \mathbf{g}_{k, l}\right\|_{L^{\infty}} & \leq\left\|\partial_{\mathbf{y}} \mathbf{g}\right\|_{L^{\infty}}, & & \left\|\partial_{\mathbf{x}, \mathbf{v}} \mathbf{g}_{k, l}\right\|_{L^{\infty}} \leq \operatorname{const}\left(\nabla \rho_{k}, \nabla \check{\rho}_{l}\right) \cdot\|\mathbf{g}\|_{L^{\infty}}, \\
\mathbf{g}_{k, l}(\mathbf{x}, \mathbf{v}, \mathbf{y}) & \in \overline{\operatorname{co}} T_{Y}(\mathbf{y}) & & \text { for all } \mathbf{x} \in \mathbb{R}^{n}, \mathbf{v} \in V, \mathbf{y} \in \bar{Y} .
\end{aligned}\right.
$$

Furthermore, $\quad \lim _{k, l \rightarrow \infty}\left\|\mathbf{g}_{k, l}-\mathbf{g}\right\|_{L^{2}\left(\mathbb{R}^{n} \times V \times \bar{Y}, \mathbb{R}^{d}\right)}=0$.

The proof of Lemma 7.6 uses only the standard properties of convolution and mollifiers (as presented in $[13, \S 4.4]$, for example) and so, we skip the details here. Its key consequence is: Consider the flow $\mathfrak{Z}_{1, \mathbf{g}_{k, l}}:[0, T] \times \mathbb{R}^{n} \times V \times \bar{Y} \longrightarrow \mathbb{R}^{d}$ defined by the unique Carathéodory solutions to initial value problem (37). Then for every $t \in[0, T]$, the function $\mathfrak{Z}_{1, \mathbf{g}_{k, l}}(t, \cdot)$ : $\bar{Y} \longrightarrow \mathbb{R}^{d}$ induces a Lipschitz continuous homeomorphism and, its weak derivative satisfies all the estimates in Lemma 5.15. Furthermore Proposition 7.4 implies

$$
\mathfrak{Z}_{1, \mathbf{g}_{k, l}}\left(t, \mathbb{R}^{n} \times V \times \bar{Y}\right) \subset \mathbb{R}^{n} \times V \times \bar{Y}
$$

for every $t \in[0, T], k, l \in \mathbb{N}$. In fact, we can even conclude from the continuity of $\mathfrak{Z}_{1, \mathbf{g}_{k, l}}(t, \cdot)^{-1}$

$$
\mathfrak{Z}_{1, \mathbf{g}_{k, l}}\left(t, \mathbb{R}^{n} \times V \times Y\right) \subset \mathbb{R}^{n} \times V \times Y \text {. }
$$

Hence, if $\varphi \in C_{c}^{0}(Z)$ is any admissible test function for $d^{f}(f, g)$ (mentioned in equation (54)) then the composition $\psi:=\varphi\left(\mathfrak{Z}_{1, \mathbf{g}_{k, l}}(t, \cdot)\right)$ is also continuous with compact support in $Z$ for 
every $t \in[0, T], k, l \in \mathbb{N}$ and, it satisfies

$$
\begin{aligned}
\|\psi\|_{L^{2}(Z)} & \leq\|\varphi\|_{L^{2}(Z)} \cdot e^{\operatorname{const}\left(\left\|\operatorname{div}_{\mathbf{y}} \mathbf{g}\right\|_{L^{\infty}}\right) \cdot t} \\
\|\psi\|_{L^{\infty}(Z)} & \leq\|\varphi\|_{L^{\infty}(Z)} \leq 1, \\
\left\|\nabla_{\mathbf{y}} \psi\right\|_{L^{\infty}\left(Z, \mathbb{R}^{d}\right)} & \leq\left\|\nabla_{\mathbf{y}} \varphi\right\|_{L^{2}\left(Z, \mathbb{R}^{d}\right)} \cdot e^{\operatorname{const}(n) \cdot\left(1+\left\|\partial_{\mathbf{y}} \mathbf{g}\right\|_{L^{\infty}}\right) \cdot t}
\end{aligned}
$$

- for essentially the same reasons as in Lemma 5.17 and inequality (43).

This observation lays the basis for reusing the arguments proving Proposition 5.21 (7.),(8.) and so, we obtain the following results: For any g, $\widehat{\mathbf{g}}$ as in Lemma 7.6 and $u, \widehat{u} \in W^{1, \infty}(Z) \cap L^{2}(Z)$, $w, \widehat{w} \in L^{2}(Z)$, consider the map

$$
\breve{\vartheta^{f}} \mathbf{g}_{k, l, u, w}:[0,1] \times L^{2}\left(\mathbb{R}^{n} \times V \times \mathbb{R}^{d}\right) \longrightarrow L^{2}\left(\mathbb{R}^{n} \times V \times \mathbb{R}^{d}\right), \quad\left(t, \breve{f}_{0}\right) \longmapsto \breve{f}(t)
$$

defined by means of the unique weak solution of

$$
\partial_{t} \breve{f}+\operatorname{div}_{\mathbf{x}}(\breve{f} \mathbf{v})+\operatorname{div}_{\mathbf{y}}\left(\breve{f} \mathcal{E}_{\mathbf{y}}\left(\mathbf{g}_{k, l}\right)\right)=\mathcal{E}_{\mathbf{y}}(u) \breve{f}+w \quad \text { in }[0, t], \quad \breve{f}(0)=\breve{f}_{0},
$$

whose existence and uniqueness are stated in Propositions 5.11 and 5.7 respectively, and its counterpart $\breve{\vartheta^{f}} \widehat{\mathrm{g}}_{k, l}, \widehat{u}, \widehat{w}:[0,1] \times L^{2}\left(\mathbb{R}^{n} \times V \times \mathbb{R}^{d}\right) \longrightarrow L^{2}\left(\mathbb{R}^{n} \times V \times \mathbb{R}^{d}\right)$.

Then Proposition 5.21 provides upper estimates for

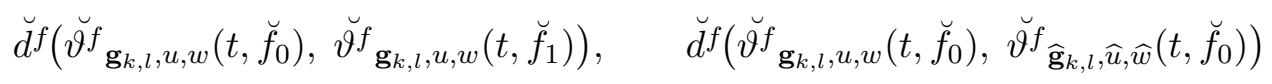

immediately. Due to the additional invariance of $Y$, however, the corresponding inequalities also hold for the metric $d^{f}$ (specified here in equation (54)), i.e. for any $k, l \in \mathbb{N}, t \in[0,1]$ and $\breve{f}_{0}, \breve{f}_{1} \in L^{2}\left(\mathbb{R}^{n} \times V \times \mathbb{R}^{d}\right)$

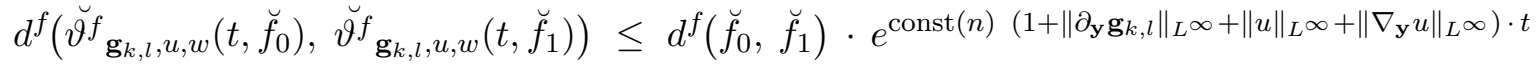

$$
\begin{aligned}
& d^{f}\left(\breve{\vartheta f}_{\mathbf{g}_{k, l}, u, w}\left(t, \breve{f}_{0}\right), \breve{\vartheta f}_{\widehat{\mathbf{g}}_{k, l}, \widehat{u}, \widehat{w}}\left(t, \breve{f}_{0}\right)\right) \leq C \cdot\left(1+\left\|\breve{f}_{0}\right\|_{L^{2}(Z)}\right) \cdot t \\
& \left(\left\|\mathbf{g}_{k, l}-\widehat{\mathbf{g}}_{k, l}\right\|_{L^{2}(Z)}+\|u-\widehat{u}\|_{L^{2}(Z)}+\|w-\widehat{w}\|_{L^{2}(Z)}\right)
\end{aligned}
$$

with a constant $C\left(n,\left\|\partial_{\mathbf{y}} \mathbf{g}_{k, l}\right\|_{L^{\infty}},\left\|\partial_{\mathbf{y}} \widehat{\mathbf{g}}_{k, l}\right\|_{L^{\infty}},\|u\|_{L^{\infty}},\left\|\nabla_{\mathbf{y}} u\right\|_{L^{\infty}},\|\widehat{u}\|_{L^{\infty}},\left\|\nabla_{\mathbf{y}} \widehat{u}\right\|_{L^{\infty}},\|\widehat{w}\|_{L^{2}}\right)$. It is worth mentioning that only information on the restrictions to $Z \stackrel{\text { Def. }}{=} \mathbb{R}^{n} \times V \times Y$ is used in these estimates although the functions are defined on $\mathbb{R}^{n} \times V \times \mathbb{R}^{d}$.

Now we can follow essentially the same way as to Corollary 3.3, i.e., via smoothing and the limit process $k, l \longrightarrow \infty$ (as in Proposition 5.23), Corollary 5.28 and the arguments in $\S 7.2$. The final conclusion is the counterpart of claimed estimate (22) proving that the weak solutions $\left.\left.\breve{f}:[0, T] \longrightarrow L^{2}\left(\mathbb{R}^{n} \times V \times \mathbb{R}^{d}\right), \breve{Q}:[0, T] \longrightarrow L^{2}\left(\mathbb{R}^{n} \times \mathbb{S}^{n-1}\right), \breve{L}:\right] 0, T\right] \longrightarrow W^{1,2}\left(\mathbb{R}^{n}\right)$ to extended problem (53) depend Lipschitz continuously on both initial states and coefficients w.r.t. the $L^{2}$ norm and the metric $d^{f}$ (restricted to $Z$ ) respectively.

As a consequence, the restriction $\left.\breve{f}(t)\right|_{Z} \in L^{2}(Z), t \in[0, T]$, is uniquely determined by the given initial state $f_{0} \in L^{2}(Z)$ and does not depend on its extension to $\mathbb{R}^{n} \times V \times \mathbb{R}^{d}$. This completes the proof of Corollary 3.4 about the case $Y \subsetneq \mathbb{R}^{d}$. 


\subsection{Proof of Corollary 3.5 about nonnegativity of components $f, L$}

For the autonomous linear transport equation (31) in the "whole space" $\mathbb{R}^{n} \times V \times \mathbb{R}^{d}$, i.e.

$$
\left\{\begin{aligned}
\partial_{t} \breve{f}+\operatorname{div}_{\mathbf{x}}(\breve{f} \mathbf{v})+\operatorname{div}_{\mathbf{y}}(\breve{f} \mathbf{g}) & =u \breve{f}+w \text { in }[0, T], \\
\breve{f}(0) & =\breve{f_{0}} \in L^{2}\left(\mathbb{R}^{n} \times V \times \mathbb{R}^{d}\right),
\end{aligned}\right.
$$

Proposition 5.18 specifies an explicit representation of the unique weak solution $\breve{f}:[0, T] \longrightarrow$ $L^{2}\left(\mathbb{R}^{n} \times V \times \mathbb{R}^{d}\right)$ whenever the autonomous coefficients satisfy $\mathrm{g} \in C_{c}^{1}\left(\mathbb{R}^{n} \times V \times \mathbb{R}^{d}, \mathbb{R}^{d}\right)$, $u \in C^{1}\left(\mathbb{R}^{n} \times V \times \mathbb{R}^{d}\right) \cap W^{1, \infty}$ and $w \in L^{2}\left(\mathbb{R}^{n} \times V \times \mathbb{R}^{d}\right)$, namely

$$
\begin{aligned}
\breve{f}(t, \xi)= & \breve{f}_{0}\left(\mathfrak{Z}_{-1, \mathbf{g}}(t ; \xi)\right) \cdot \exp \left(\int_{0}^{t}\left(u-\operatorname{div}_{\mathbf{y}} \mathbf{g}\right)\left(\mathfrak{Z}_{-1, \mathbf{g}}(s ; \xi)\right) d s\right)+ \\
& \int_{0}^{t}\left(w\left(\mathfrak{Z}_{-1, \mathbf{g}}(t-s ; \xi)\right) \cdot \exp \left(\int_{0}^{t-s}\left(u-\operatorname{div}_{\mathbf{y}} \mathbf{g}\right)\left(\mathfrak{Z}_{-1, \mathbf{g}}(r ; \xi)\right) d r\right)\right) d s .
\end{aligned}
$$

Obviously, $\breve{f}_{0} \geq 0$ and $w \geq 0$ implies $\breve{f}(t, \cdot) \geq 0$ Lebesgue-almost everywhere in $\mathbb{R}^{n} \times V \times \mathbb{R}^{d}$. Following the same steps of approximation as in $\S 5$, the corresponding implication also holds for less regular (but still autonomous) coefficients $\mathbf{g} \in L^{\infty}\left(\mathbb{R}^{n} \times V \times \mathbb{R}^{d}, \mathbb{R}^{d}\right) \cap L^{2}, \partial_{\mathbf{y}} \mathbf{g} \in L^{\infty}, u \in$ $L^{\infty}\left(\mathbb{R}^{n} \times V \times \mathbb{R}^{d}\right) \cap L^{2}, \partial_{\mathbf{y}} u \in L^{\infty}, w \in L^{2}\left(\mathbb{R}^{n} \times V \times \mathbb{R}^{d}\right)$ - as a consequence of Proposition 5.21 (8.) and the following lemma:

Lemma 7.7 Let $\left(f_{k}\right)_{k \in \mathbb{N}}$ be any bounded sequence in $L^{2}(Z)$ which converges to $f \in L^{2}(Z)$ w.r.t. $d^{f}$ and satisfies $f_{k} \geq 0$ a.e. in $Z$ for every $k \in \mathbb{N}$. Then, $f \geq 0$ a.e. in $Z$.

Proof. Assume that the claim does not hold, i.e., there is a set $A \subset Z$ of positive Lebesgue measure with $f<0$ in $A$. Then, for some sufficiently small $\varepsilon>0$, there exists a subset $\widehat{A}$ of $A$ which has Lebesgue measure $\mu_{\widehat{A}}>0$ and fulfils $f \leq-\varepsilon$ in $\widehat{A}$ additionally.

Set $M:=\sup \left\{\left\|f_{k}\right\|_{L^{2}(Z)},\|f\|_{L^{2}(Z)} \mid k \in \mathbb{N}\right\}<\infty$ and let $\chi_{\widehat{A}}: Z \longrightarrow\{0,1\}$ denote the characteristic function of $\widehat{A} \subset Z$. It is Lebesgue integrable and so, there is some $\varphi \in C_{c}^{\infty}(Z)$ with

$$
\left\|\varphi-\chi_{\widehat{A}}\right\|_{L^{2}(Z)} \leq \frac{\varepsilon \mu_{\widehat{A}}}{4 M}
$$

Finally using the abbreviation $C:=\frac{1}{1+\|\varphi\|_{L^{2}}+\|\varphi\|_{L^{\infty}+\left\|\nabla_{\mathbf{y}} \varphi\right\|_{L^{\infty}}}}$, we obtain for every $k \in \mathbb{N}$

$$
\begin{aligned}
d^{f}\left(f_{k}, f\right) & \geq C \cdot \int_{Z} \varphi \cdot\left(f_{k}-f\right) d \mathbf{z} \\
& \geq C\left(\int_{Z} \chi_{\widehat{A}} \cdot\left(f_{k}-f\right) d \mathbf{z}-\left\|\varphi-\chi_{\widehat{A}}\right\|_{L^{2}(Z)} \cdot\left\|f_{k}-f\right\|_{L^{2}(Z)}\right) \\
& \geq C\left(\varepsilon \cdot \mu_{\widehat{A}}-\frac{\varepsilon \mu_{\widehat{A}}}{4 M} \cdot 2 M\right)>0
\end{aligned}
$$

This contradicts the assumption $d^{f}\left(f_{k}, f\right) \longrightarrow 0$ for $k \longrightarrow \infty$.

Due to these arguments about "preserving" the sign while converging w.r.t. $d^{f}$, Proposition 5.26 about the nonautonomous linear problem in the "whole space" $\mathbb{R}^{n} \times V \times \mathbb{R}^{d}$

$$
\left\{\begin{aligned}
\partial_{t} \breve{f}+\operatorname{div}_{\mathbf{x}}(\breve{f} \mathbf{v})+\operatorname{div}_{\mathbf{y}}(\breve{f} \widetilde{\mathbf{g}}(t)) & =\widetilde{u}(t) f+\widetilde{w}(t) \quad \text { in }[0, T], \\
\breve{f}(0) & =\breve{f} 0 \in L^{2}\left(\mathbb{R}^{n} \times V \times \mathbb{R}^{d}\right)
\end{aligned}\right.
$$


can be extended by the following statement:

If $\breve{f}_{0} \geq 0$ and if $\widetilde{w} \in L^{\infty}\left(0, T ; L^{2}\left(\mathbb{R}^{n} \times V \times \mathbb{R}^{d}\right)\right)$ satisfies $\widetilde{w}(t) \geq 0$ for almost every $t \in[0, T]$, then the unique weak solution $\breve{f}:[0, T] \longrightarrow L^{2}\left(\mathbb{R}^{n} \times V \times \mathbb{R}^{d}\right)$ fulfils $\breve{f}(t) \geq 0$ for every $t \in[0, T]$.

Finally both the method of successive approximation used in $\S 7.1$ and the potential restriction to $Z \stackrel{\text { Def. }}{=} \mathbb{R}^{n} \times V \times Y$ (if $Y \subsetneq \mathbb{R}^{d}$ as in $\S 7.2$ ) also preserve nonnegativity. Hence the proof for the component $f:[0, T] \longrightarrow L^{2}(Z)$ of the unique weak solution to the original problem (20) is completed: $f(t) \geq 0$ holds for every $t \in[0, T]$.

In regard to the component $L:] 0, T] \longrightarrow W^{1,2}\left(\mathbb{R}^{n}\right)$, this observation implies

$$
\sigma(t, \cdot):=\beta_{L} \cdot L(t)+\mathcal{R}(t, f(t), Q(t), L(t)) \geq 0 \quad \text { Lebesgue-a.e. in } \mathbb{R}^{n}
$$

for Lebesgue-almost every $t \in[0, T] . \quad L$ is the weak solution to the nonautonomous linear reaction-diffusion problem (52) (with constant parameters $\kappa:=\kappa_{L}>0, \beta:=\beta_{L} \geq 0$ ) and, the explicit representation $(51)$ of $L(t, \cdot): \mathbb{R}^{n} \longrightarrow \mathbb{R}$ reveals $L(t, \cdot) \geq 0$ directly.

\subsection{Proof of Corollary 3.7 about nonnegative solutions to model problem (23)}

In model (23) for cell migration through tissue, the uniquely determined component $Q$ : $[0, T] \longrightarrow L^{2}\left(\mathbb{R}^{n} \times \mathbb{S}^{n-1}\right)$ satisfies the ordinary differential equation (with all values in a Banach space)

$$
\begin{aligned}
\partial_{t} Q(t) & =\mathcal{T}(t, f(t), Q(t), L(t)) \\
\Longrightarrow \partial_{t} Q(t, \mathbf{x}, \theta) & =r_{\mathrm{ECM}} \int_{Y} \int_{V}\left(\left|\theta \cdot \hat{\mathbf{v}}^{\prime}\right|-1\right) f\left(t, \mathbf{x}, \mathbf{v}^{\prime}, \mathbf{y}^{\prime}\right) d \mathbf{v}^{\prime} d \mathbf{y}^{\prime} \cdot \frac{Q(t, \mathbf{x}, \theta)}{1+\gamma_{Q} \cdot|Q(t, \mathbf{x}, \theta)|}
\end{aligned}
$$

for Lebesgue-almost every $(\mathbf{x}, \theta) \in \mathbb{R}^{n} \times \mathbb{S}^{n-1}$ fixed and then each $t \in[0, T]$. Here the component $f:[0, T] \longrightarrow L^{2}(Z)$ is bounded and so, $f \in L^{2}([0, T] \times Z)$. Fubini's theorem implies $f(\cdot, \mathbf{x}, \cdot, \cdot) \in L^{2}([0, T] \times V \times Y)$ for Lebesgue-almost every $\mathbf{x} \in \mathbb{R}^{n}$.

Thus for Lebesgue-almost every $(\mathbf{x}, \theta) \in \mathbb{R}^{n} \times \mathbb{S}^{n-1}$, there exists a scalar auxiliary function $\eta_{\mathbf{x}, \theta} \in L^{2}([0, T]) \subset L^{1}([0, T])$ with

$$
\left|\partial_{t} Q(t, \mathbf{x}, \theta)\right| \leq \eta_{\mathbf{x}, \theta}(t) \cdot \frac{|Q(t, \mathbf{x}, \theta)|}{1+\gamma_{Q} \cdot|Q(t, \mathbf{x}, \theta)|} \leq \eta_{\mathbf{x}, \theta}(t) \cdot|Q(t, \mathbf{x}, \theta)| .
$$

Gronwall's inequality guarantees $Q(\cdot, \mathbf{x}, \theta)=0$ in $\left[t_{0}, T\right]$ whenever there is a time instant $t_{0} \in$ $\left[0, T\left[\right.\right.$ with $Q\left(t_{0}, \mathbf{x}, \theta\right)=0$. Hence we conclude from the continuity of $Q(\cdot, \mathbf{x}, \theta):[0, T] \longrightarrow \mathbb{R}$ that $Q_{0}(\mathbf{x}, \theta) \geq 0$ implies $Q(\cdot, \mathbf{x}, \theta) \geq 0$ in $[0, T]$.

Finally preserving nonnegativity of components $f(t), L(t)$ at every time $t \in] 0, T]$ is an immediate consequence of Corollary 3.5 since sign assumption $(f)$ here implies condition (vii) there. Thus the proof of Corollary 3.7 is completed. 


\section{References}

[1] Ambrosio, L. (2008): Transport Equation and Cauchy Problem for Non-Smooth Vector Fields, in: Dacorogna, B. \& Marcellini, P. (Eds.), Calculus of Variations and Nonlinear Partial Differential Equations, Springer, Lecture Notes in Mathematics 1927, pp.1-41

[2] Ambrosio, L. (2004): Transport equation and Cauchy problem for BV vector fields, Invent. Math. 158 (2004), no.2, pp.227-260

[3] Ambrosio, L. \& Bernard, P. (2008): Uniqueness of signed measures solving the continuity equation for Osgood vector fields, Rend. Lincei Mat. Appl. 19 (2008), pp.237-245

[4] Ambrosio, L. \& Crippa, G. (2008): Existence, uniqueness, stability and differentiability properties of the flow associated to weakly differentiable vector fields, in: Ambrosio, L. et al., Transport equations and multi-D hyperbolic conservation laws, pp.3-57, Lect. Notes Unione Mat. Ital. 5, Springer, 2008.

[5] Ambrosio, L., Gigli, N. \& Savaré, G. (2005): Gradient Flows in Metric Spaces and in the Space of Probability Measures. Lectures in Mathematics ETH Zürich. Birkhäuser, 2005.

[6] Anderson A.R.A, Chaplain M.A.J, Newman E.L, Steele R.J.C \& Thompson A.M (2000): Mathematical modeling of tumor invasion and metastasis, J. Theoretical Med. 2 (2000), pp.129-154.

[7] Aubin, J.-P. \& Frankowska, H. (1990): Set-Valued Analysis, Birkhäuser, 1990.

[8] Barocas, V. H. \& Tranquillo, R.T. (1997): An Anisotropic Biphasic Theory of TissueEquivalent Mechanics: The Interplay Among Cell Traction, Fibrillar Network Deformation, Fibril Alignment and Cell Contact Guidance, ASME Journal of Biomechanical Engineering 119 (1997), pp.137-145.

[9] Bellomo, N., Bellouquid, A., Nieto, J. \& Soler, J. (2010): Complexity and mathematical tools toward the modeling of multicellular growing systems, Math. Comput. Modeling 51 (2010), pp.441-451.

[10] Berry, H. (1999): Oscillatory behavior of a simple kinetic model for proteolysis during cell invasion, Biophys. J. 77 (1999), pp.655-665.

[11] Bouchut, F. \& Crippa, G. (2006): Uniqueness, renormalization and smooth approximations for linear transport equations, SIAM J. Math. Anal. 38 (2006), pp.1316-1328

[12] Bournaveas, N. \& Calvez, V. (2008): Global existence for the kinetic chemotaxis model without pointwise memory effects, and including internal variables, Kinet. Relat. Models 1 (2008), pp.29-48.

[13] Brezis, H. (2011): Functional Analysis, Sobolev Spaces and Partial Differential Equations. Universitext. Springer, New York, 2011. 
[14] Burenkov, V.I. (1998): Sobolev Spaces on Domains. Teubner-Texte zur Mathematik 137. Teubner, Stuttgart, 1998.

[15] Chalub, F., Markowich, P.A., Perthame, B. \& Schmeiser, C. (2004): Kinetic models for chemotaxis and their drift-diffusion limits, Monatsh. Math. 142 (2004), pp.123-141.

[16] Chaplain, M. A. J. \& Lolas, G. (2006): Mathematical modelling of cancer invasion of tissue: dynamic heterogeneity. Netw. Heterog. Media 1 (2006), pp.399-439.

[17] Chauvière, A., Hillen, T. \& Preziosi, L. (2010): Modeling cell movement in anisotropic and heterogeneous network tissues, Networks heterogeneous media 2 (2007) 333-357.

[18] Condeelis, J. \& Segall, J.E. (2003): Intravital imaging of cell movement in tumors, Nat. Rev. Cancer 3 (2003), pp.921-930.

[19] DiPerna, R. J. \& Lions, P.-L. (1989): Ordinary differential equations, transport theory and Sobolev spaces, Invent. Math. 98 (1989), pp.511-547.

[20] Erban, R. \& Othmer, H.G. (2005): From signal transduction to spatial pattern formation in E. coli: A paradigm for multi-scale modeling in biology, Multiscale Modeling and Simulation 3 (2005), pp.362-394.

[21] Erban, R. \& Hwang, H.J. (2006): Global Existence Results for Complex Hyperbolic Models of Bacterial Chemotaxis, Discrete Contin. Dyn. Syst. Ser. B 6 (2006), pp.12391260 .

[22] Evans, L.C. (1998): Partial differential equations, Graduate Studies in Mathematics 19, Amer. Math. Soc. Providence, 1998.

[23] Fidler, I.J. (2003): The pathogenesis of cancer metastasis: the 'seed and soil' hypothesis revisited, Nat. Rev. Cancer 3 (2003), pp.453-458.

[24] Filbet, F., Laurencot, P. \& Perthame, B. (2005): Derivation of Hyperbolic Models for Chemosensitive Movement, J. Math. Biology 50 (2005), pp.189-207.

[25] Firmani, B., Guerri, L. \& Preziosi, L. (1999): Tumor immune system competition with medically induced activation disactivation, Math. Models Meth. Appl. Sci. 9 (1999), pp.491-512.

[26] Frankowska, H., Plaskacz, S. \& Rzeżuchowski, T. (1995): Measurable viability theorems and the Hamilton-Jacobi-Bellman equation, J. Differ. Equations 116 (1995), pp.265-305.

[27] Frankowska, H. (1990): A priori estimates for operational differential inclusions, J. Differ. Equations 84 (1990), pp.100-128

[28] Friedl, P. \& Wolf, K. (2003): Tumour-cell invasion and migration: diversity and escape mechanisms, Nature Rev. Cancer 3 (2003), pp.362-374. 
[29] Gianelli, G., Falk-Marzillier, J., Schiraldi, O., Stetler-Stevenson, W.G. \& Quaranta, V. (1997): Induction of cell migration by matrix metalloprotease-2 cleavage of laminin-5, Science 277 (1997), pp.225-228.

[30] Hartman, Ph. (2002): Ordinary Differential Equations, second edition, SIAM Classics in Applied Mathematics, 2002.

[31] Hille, E. \& Phillips, R.S. (1957): Functional Analysis and Semi-groups, revised edition, AMS Colloquium Publications 31. Amer. Math. Soc. Providence, 1957.

[32] Hillen, T. (2006): Mesoscopic and macroscopic models for mesenchymal motion, J. Math. Biol. 53 (2006), pp.585-616.

[33] Hillen, T., Hinow, P. \& Wang, Z. (2010): Mathematical analysis of a kinetic model for cell movement in network tissues, Discrete Contin. Dyn. Syst. Ser. B 14 (2010), pp.1055-1080.

[34] Hofmann, U.B., Westphal, J.R., van Muijen, G.N. \& Ruiter, D.J. (2000): Matrix metalloproteinases in human melanoma, J. Invest. Dermatol. 115 (2000), pp.337-344.

[35] Kelkel, J. \& Surulescu, C. (2011): On some models for cancer cell migration through tissue networks, Math. Biosci. Eng. 8 (2011), pp.575-589.

[36] Kelkel, J. \& Surulescu, C. (2012): A multiscale approach to cell migration in tissue networks, Math. Models Methods Appl. Sci. 22 (2012), no.3, 1150017.

[37] Kessenbrock, K., Plaks, V. \& Werb, Z. (2010): Matrix metalloproteinases: regulators of the tumor microenvironment, Cell 141 (2010), pp.52-67.

[38] Lang, S. (1993): Real and Functional Analysis, third edition. Graduate Texts in Mathematics 142, Springer, 1993.

[39] Lauffenburger, D. (1989): A simple model for the effects of receptor-mediated cellsubstratum adhesion on cell migration, Chem. Eng. Sci. 44 (1989), pp.1903-1914.

[40] Le Bris, C. \& Lions, P.-L. (2004): Renormalized solutions of some transport equations with partially $W^{1,1}$ velocities and applications, Ann. Mat. Pura Appl. (4) 183 (2004), pp. $97-130$

[41] Lorenz, Th. (2010): Mutational Analysis. A Joint Framework for Cauchy Problems in and beyond Vector Spaces. Lecture Notes in Mathematics 1996, Springer, 2010.

[42] Maghazachi, A.A. (2000): Intracellular signaling events at the leading edge of migrating cells, Int. J. Biochem. Cell Biol. 32 (2000), pp.931-943.

[43] Maini, P.K. (1989): Spatial and spatio-temporal patterns in a cell-haptotaxis model, $J$. Math. Biol. 27 (1989), pp.507-522. 
[44] Mallet, D.G. \& Pettet, G.J. (2006): A mathematical model for integrin-mediated haptotactic cell migration, Bull. Math. Biol. 68 (2006), pp.231-253.

[45] Maniglia, St. (2007): Probabilistic representation and uniqueness results for measurevalued solutions of transport equations, J. Math. Pures Appl. (9) 87 (2007), no.6, pp.601626 .

[46] Meral, G. \& Surulescu, C. (2011): Mathematical modelling, analysis and numerical simulations for the influence of the heat shock proteins on tumour invasion, Preprint ICAM, WWU Münster (submitted).

[47] Mucha, P. (2010): Transport equation: Extension of classical results for $\operatorname{div} b \in$ BMO, J. Differential Equations 249 (2010), pp.1871-1883

[48] Nagumo, M. (1942): Über die Lage der Integralkurven gewöhnlicher Differentialgleichungen, Proc. Phys.-Math. Soc. Japan, III. Ser. 24 (1942), pp.551-559

[49] Othmer, H.G., Dunbar, S. \& Alt, W. (1988): Models of dispersal in biological systems, J. Math. Biol. 26 (1988), pp.263-298.

[50] Othmer, H.G. \& Hillen, T. (2002): The diffusion limit of transport equations II: Chemotaxis equations, SIAM J. Applied Math. 62 (2002), pp.1222-1250.

[51] Rudolph-Owen, L.A., Chan, R., Muller, W.J. \& Matrisian, L.M. (1998): The matrix metalloproteinase matrilysin influences early-stage mammary tumorigenesis, Cancer Res. 58 (1998), pp.5500-5506.

[52] Sameni, M., Moin, K. \& Sloane, B.F. (2001): Imaging proteolysis by living human breast cancer cells, Neoplasia 2 (2001), pp.496-504.

[53] Stroock, D. (1974): Some stochastic processes which arise from a model of the motion of a bacterium, Z. Wahrscheinlichkeitstheorie und verw. Gebiete 28 (1974), pp.305-315.

[54] Surulescu, C. \& Surulescu, N. (2010): On two approaches to a multiscale model for bacterial chemotaxis, Preprint IANS, University of Stuttgart (submitted).

[55] Surulescu, C. \& Surulescu, N. (2010): A nonparametric approach to cell dispersal, Int. J. of Biomathematics and Biostatistics 1 (2010), pp.109-128.

[56] Surulescu, C. \& Surulescu, N. (2011): Modeling and simulation of bacterial motion via a nonparametric method, Math. Biosci. Eng., 8 (2011), pp.263-277.

[57] Szymanska, Z., Urbanski, J. \& Marciniak-Czochra, A. (2009): Mathematical modelling of the influence of heat shock proteins on cancer invasion of tissue, J. Math. Biol. 58 (2009), pp.819-844.

[58] Talmadge, J.E. \& Fidler, I.J. (2010): AACR centennial series: the biology of cancer metastasis: historical perspective, Cancer Res. 70, pp.5649-5669. 
[59] Tolstonogov, A.A. (1992): Solutions of evolutions inclusions I, Siberian Math. J. 33 (1992), no.3, pp.500-511

[60] Tosin, A. \& Preziosi, L. (2010): Multiphase modeling of tumor growth with matrix remodeling and fibrosis, Mathematical and Computer Modelling 52 (2010), pp.969-976.

[61] Traqui, P. (1995): From passive diffusion to active cellular migration in mathematical models of tumour invasion, Acta Biotheoretica 43 (1995), pp.443-464.

[62] Ursescu, C. (1986): Carathéodory solutions of ordinary differential equations on locally closed sets in finite-dimensional spaces, Math. Japon. 31 (1986), pp.483-491

[63] Villani, C. (2009): Optimal Transport - Old and New, Grundlehren der mathematischen Wissenschaften 338, Springer, 2009.

[64] Yosida, K. (1980): Functional Analysis (sixth edition), Grundlehren der mathematischen Wissenschaften 123, Springer, 1980.

[65] Ziemer, W. (1989): Weakly Differentiable Functions. Sobolev Spaces and Functions of Bounded Variations, Graduate Texts in Mathematics 120, Springer, 1989. 